Prepared for the U.S. Department of Energy

under Contract DE-AC05-76RL01830

\title{
Ship Effect Neutron Measurements and Impacts on Low-Background Experiments
}

Estanislao Aguayo

Richard T. Kouzes

Edward R. Siciliano

October 2013

Pacific Northwest

NATIONAL LABORATORY

Proudly Operated by Battelle Since 1965 


\title{
DISCLAIMER
}

This report was prepared as an account of work sponsored by an agency of the United States Government. Neither the United States Government nor any agency thereof, nor Battelle Memorial Institute, nor any of their employees, makes any warranty, express or implied, or assumes any legal liability or responsibility for the accuracy, completeness, or usefulness of any information, apparatus, product, or process disclosed, or represents that its use would not infringe privately owned rights. Reference herein to any specific commercial product, process, or service by trade name, trademark, manufacturer, or otherwise does not necessarily constitute or imply its endorsement, recommendation, or favoring by the United States Government or any agency thereof, or Battelle Memorial Institute. The views and opinions of authors expressed herein do not necessarily state or reflect those of the United States Government or any agency thereof.

\author{
PACIFIC NORTHWEST NATIONAL LABORATORY \\ operated by \\ BATTELLE \\ for the \\ UNITED STATES DEPARTMENT OF ENERGY \\ under Contract DE-AC05-76RL01830
}

Printed in the United States of America
Available to DOE and DOE contractors from the
Office of Scientific and Technical Information,
P.O. Box 62, Oak Ridge, TN 37831-0062;
ph: (865) 576-8401
fax: (865) 576-5728
email: reports@adonis.osti.gov

Available to the public from the National Technical Information Service, U.S. Department of Commerce, 5285 Port Royal Rd., Springfield, VA 22161

ph: (800) 553-6847

fax: $(703) 605-6900$

email: orders@ntis.fedworld.gov

online ordering: http://www.ntis.gov/ordering.htm 


\section{Ship Effect Neutron Measurements and Impacts on Low-Background Experiments}

Estanislao Aguayo

Richard T. Kouzes

Edward R. Siciliano

October 2013

Pacific Northwest National Laboratory

Richland, Washington 99352 


\begin{abstract}
The primary particles entering the upper atmosphere as cosmic rays create showers in the atmosphere that include a broad spectrum of secondary neutrons, muons and protons. These cosmic ray secondaries interact with materials at the surface of the Earth, yielding prompt backgrounds in radiation detection systems, as well as inducing long-lived activities through spallation events, dominated by the higher-energy neutron secondaries. For historical reasons, the multiple neutrons produced in spallation cascade events are referred to as "ship effect" neutrons. Quantifying the background from cosmic ray induced activities is important to lowbackground experiments, such as neutrinoless double-beta decay.

Since direct measurements of the effects of shielding on the cosmic ray neutron spectrum are not available, Monte Carlo modeling is used to compute such effects. However, there are large uncertainties (orders of magnitude) in the possible cross-section libraries and the cosmic ray neutron spectrum for the energy range needed in such calculations.

The measurements reported here were initiated to validate results from Monte Carlo models through experimental measurements in order to provide some confidence in the model results.

The results indicate that the models provide the correct trends of neutron production with increasing density, but there is substantial disagreement between the models and experimental results for the lower-density materials of $\mathrm{Al}, \mathrm{Fe}$ and $\mathrm{Cu}$.
\end{abstract}




\section{Acronyms and Abbreviations}

A

$\mathrm{ADC}$

cps

DOE

$\varepsilon$

GE

HDPE

INC

MCNP

PNNL

$\mathrm{R}+\mathrm{A}$

TTL

VME
Atomic number

Analog to digital converter

Counts per second

U.S. Department of Energy

Detection efficiency

General Electric

High Density Polyethylene

Inter-nuclear cascade

Monte Carlo N-Particle

Pacific Northwest National Laboratory

Reals plus accidentals analysis method

Transistor-transistor logic

Versa Module Eurocard 


\section{Contents}

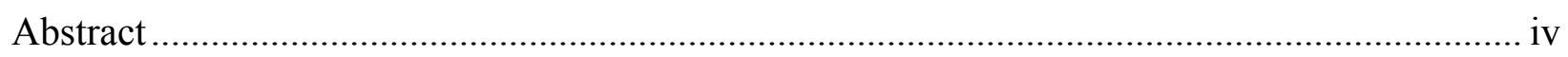

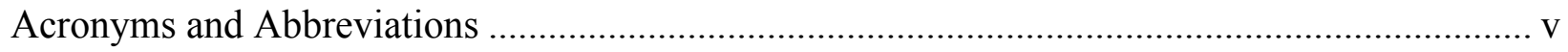

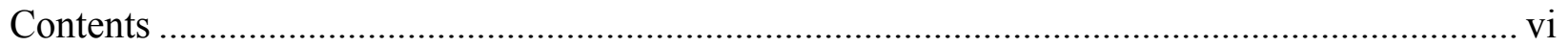

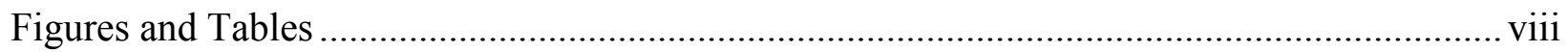

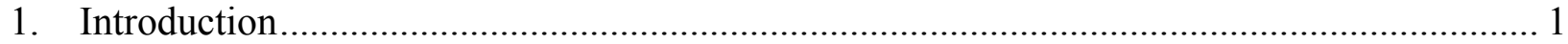

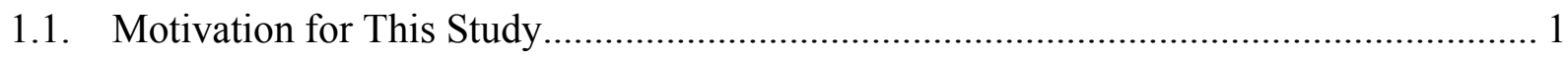

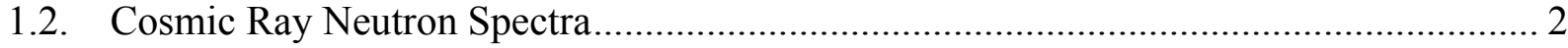

1.3. Neutron Cross-Sections .................................................................................... 3

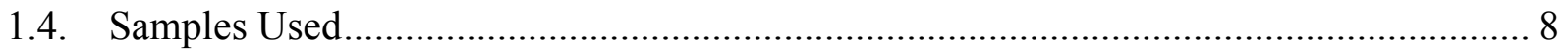

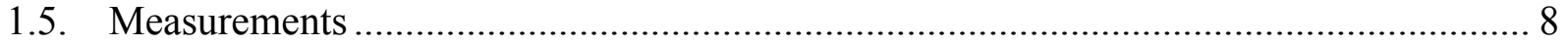

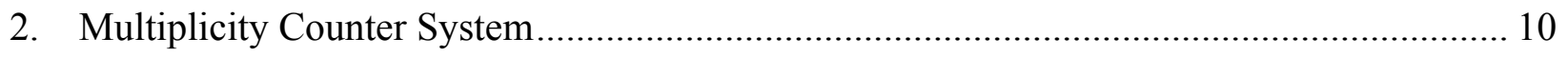

3. Neutron Efficiency and Gamma Ray Sensitivity Measurements ..................................... 15

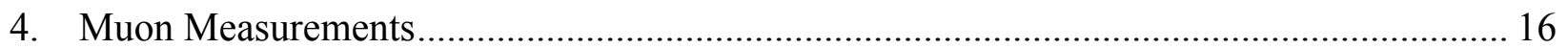

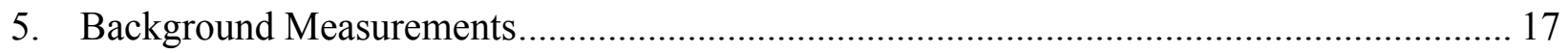

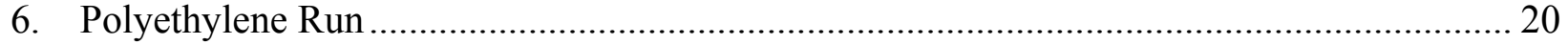

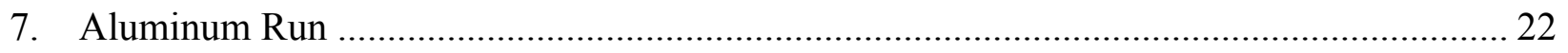

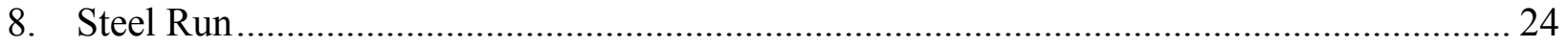

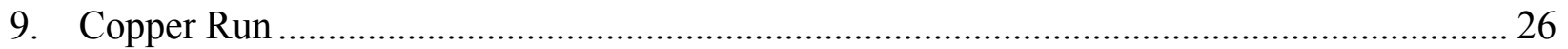

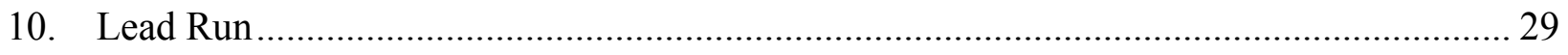

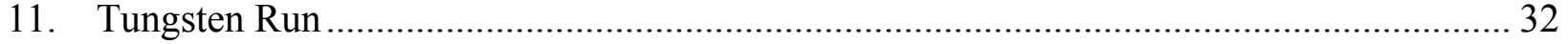

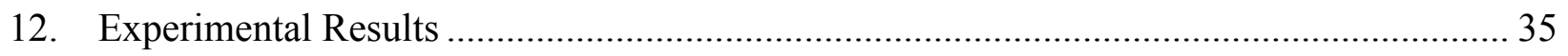

12.1. Integrated Experimental Multiplicity Counts ....................................................... 35

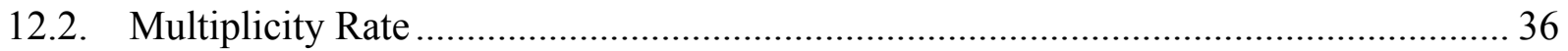

12.3. Reals and Accidentals Multiplicity Analysis ....................................................... 38

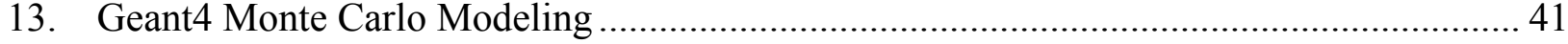

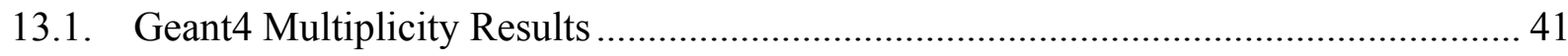

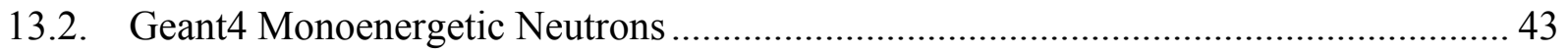

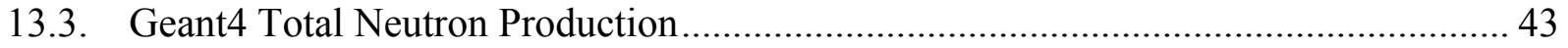


13.4. Geant4 Outgoing Neutron Energy Spectra ................................................................ 45

13.5. Geant4 Incoming Cosmic Neutron Energy Spectra........................................................ 46

14. MCNPX Monte Carlo Model Comparisons ……………………………………….......... 47

14.1. MCNP Monoenergetic Neutron Results .................................................................. 47

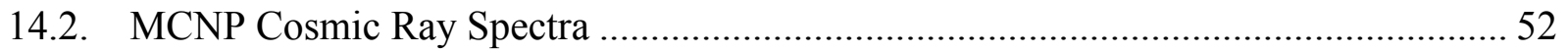

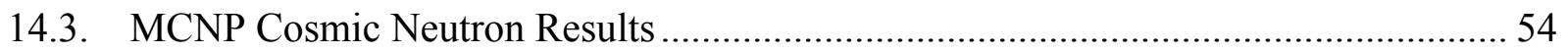

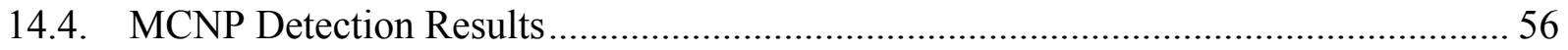

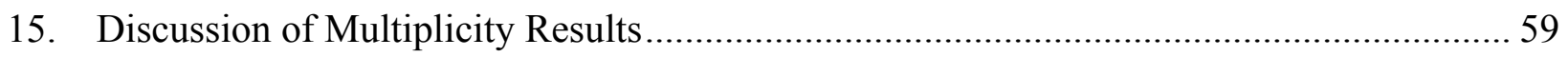

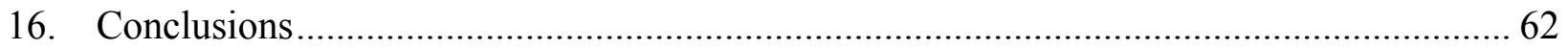

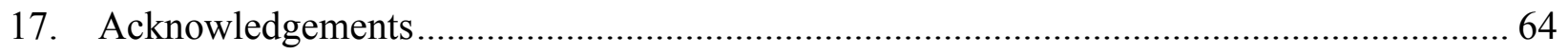

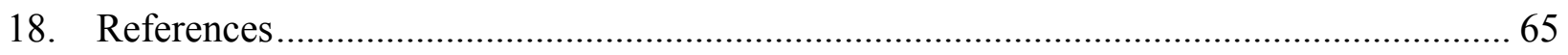




\section{Figures and Tables}

\section{Figures}

Figure 1-1. Cutaway view of GERDA shield design............................................................. 1

Figure 1-2. Cosmic ray neutron energy spectra from $1 \mathrm{MeV}$ to $1 \mathrm{GeV}$ from various authors....... 3

Figure 1-3. Differential neutron cross-section example. ..................................................... 4

Figure 1-4. Total cross section for neutron processes in polyethylene used in Geant 4 ................ 5

Figure 1-5. Total cross section for neutron processes in Al used in Geant4 ............................ 5

Figure 1-6. Total cross section for neutron processes in Fe used in Geant4 ............................. 6

Figure 1-7. Total cross section for neutron processes in $\mathrm{Cu}$ used in Geant 4 ............................. 6

Figure 1-8. Total cross section for neutron processes in $\mathrm{Pb}$ used in Geant4 ............................ 7

Figure 1-9. Total cross section for neutron processes in $\mathrm{W}$ used in Geant 4 .............................. 7

Figure 2-1. Experimental set-up of the multiplicity counter and electronics at the 3440 building.

Figure 2-2. Top view of the multiplicity counter (with polyethylene sample)........................ 11

Figure 2-3. Side and top view of the multiplicity counter in the MCNP model....................... 13

Figure 2-4. Energy and position efficiency profiles for 16-tube coincidence counter used........ 14

Figure 4-1. Cosmic ray fluctuation in a 35 year time period, from [Ziegler 1998] .................... 16

Figure 4-2. The uWitness detector [Aguayo 2012a] ..................................................... 16

Figure 5-1. Distribution of neutron multiplicity events caused by cosmic neutrons without a

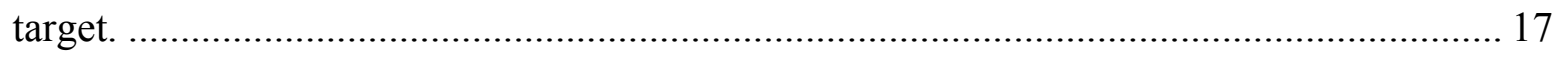

Figure 5-2. Background rates recorded before and after the runs using the different materials as a

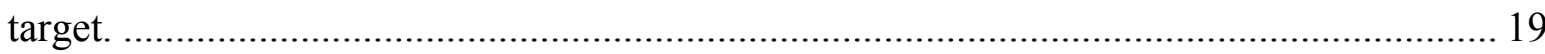

Figure 6-1. Multiplicity counter open showing the HDPE in position for measurement............ 20

Figure 6-2. Distribution of neutron multiplicity caused by cosmic neutrons in polyethylene. .... 21

Figure 7-1. Multiplicity counter open showing the aluminum in position for measurement ....... 22

Figure 7-2. Distribution of neutron multiplicity caused by cosmic neutrons in aluminum......... 23

Figure 8-1. Multiplicity counter open showing the iron in position for measurement............... 24

Figure 8-2. Distribution of neutron multiplicity caused by cosmic neutrons in steel................ 25

Figure 9-1. Multiplicity counter open showing the copper in position for measurement............ 26

Figure 9-2. Distribution of neutron multiplicity caused by cosmic neutrons in copper............. 27

Figure 10-1. Multiplicity counter open showing the lead in position for measurement.............. 29 
Figure 10-2. Distribution of neutron multiplicity caused by cosmic neutrons in lead. 30

Figure 10-3. Distribution of neutron multiplicity caused by cosmic neutrons in lead up to 50 ... 31

Figure 11-1. Multiplicity counter open showing the tungsten in position for measurement........ 32

Figure 11-2. Distribution of neutron multiplicity caused by cosmic neutrons in tungsten.......... 33

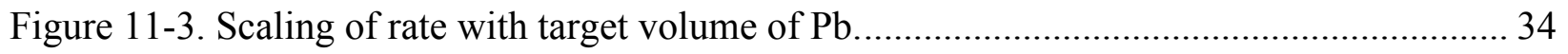

Figure 12-1. Integrated number of events versus neutron density in 10 ms gate length. ............ 35

Figure 12-2. Integrated number of events versus neutron density in $1 \mathrm{~ms}$ gate length. ............... 35

Figure 12-3. Event rate versus neutron density for three multiplicities................................. 37

Figure 12-4. Event rate versus neutron density from [Kouzes 2008] ..................................... 38

Figure 12-5. Measured neutron multiplicities for the materials studied................................. 39

Figure 12-6. Comparison of neutron triples and quads from $\mathrm{R}+\mathrm{A}$ analysis for the materials

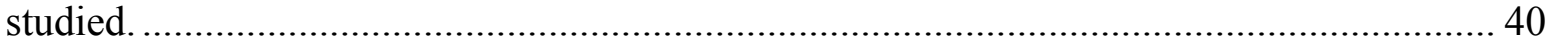

Figure 13-1. Cosmic neutron simulation results for the materials studied. .............................. 42

Figure 13-2. Cosmic neutron simulation results for low-Z materials..................................... 42

Figure 13-3. Simulated neutrons out from a cubic foot of material as a function of neutron

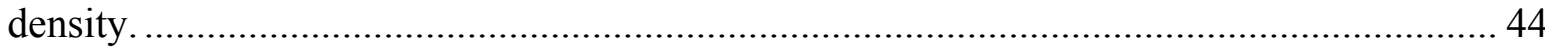

Figure 13-4. Simulated neutrons out from a cubic foot of material as a function of neutron

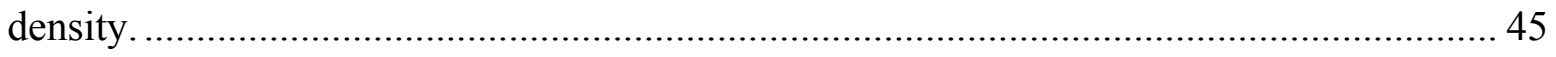

Figure 13-5. Spectra computed with Geant4 for neutrons exiting each of the materials............. 46

Figure 14-1. MCNP results for total neutrons out at several energies for various materials....... 48

Figure 14-2. MCNP results for $1 \mathrm{GeV}$ and $100 \mathrm{MeV}$ monoenergetic neutrons on various

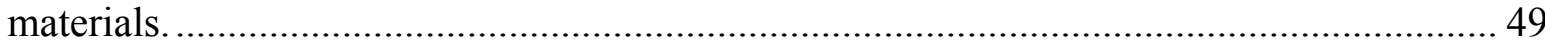

Figure 14-3. MCNP results for $10 \mathrm{MeV}$ and $1 \mathrm{MeV}$ monoenergetic neutrons on various materials.

Figure 14-4. Simulation of collision points and cascade tracks for $1 \mathrm{GeV}$ (left) and $100 \mathrm{MeV}$

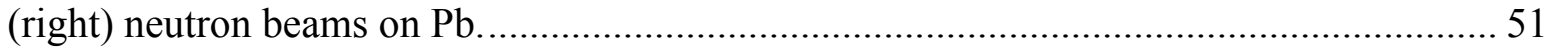

Figure 14-5. MCNP incident cosmic ray neutron spectra................................................ 52

Figure 14-6. MCNP incident cosmic ray proton spectra. .................................................... 53

Figure 14-7. MCNP incident cosmic ray muon spectra....................................................... 53

Figure 14-8. MCNP results for cosmic ray components for various materials. ........................ 55

Figure 14-9. MCNPX and Geant4 results for total neutrons out for different materials............. 55

Figure 14-10. Cutaway view of the MCNPX detector model: $\mathrm{Pb}$ (left) and $\mathrm{Cu}$ (right). ............. 56 
Figure 14-11. MCNPX results versus neutron density for neutrons out and neutrons detected with

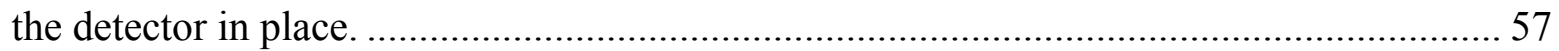

Figure 14-12. MCNPX results versus atomic mass for neutrons out and neutrons detected with the detector in place. 58

Figure 15-1. Comparison of simulation to measurement for counts greater than Poisson in a $1 \mathrm{~ms}$ window. 59

Figure 15-2. Comparison of $\mathrm{R}+\mathrm{A}$ analysis of measurements to simulation of counts greater than Poisson in a $1 \mathrm{~ms}$ window. 60

\section{Tables}

Table 1.1. Sample masses and volumes used in the runs.................................................... 8

Table 1.2. Measurements made as part of this study. ............................................................ 9

Table 3.1. Source run parameters from VME system (November 28, 2012) ........................... 15

Table 3.2. Source run parameters from VME system (December 10, 2012)........................... 15

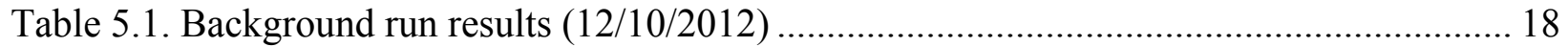

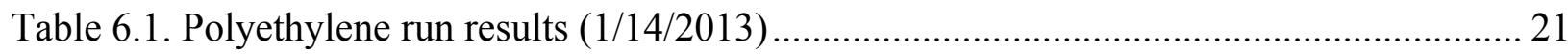

Table 7.1. Aluminum run results $(3 / 1 / 2013)$......................................................................... 23

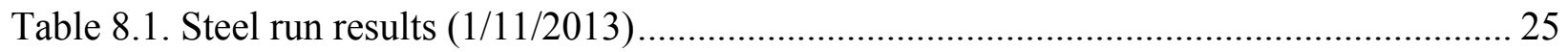

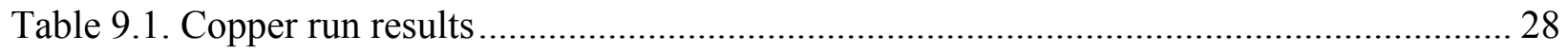

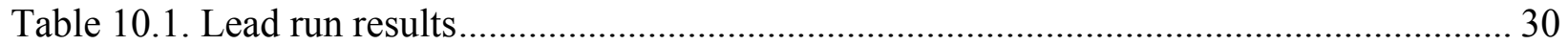

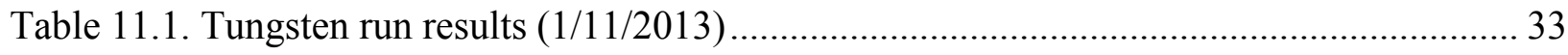

Table 12.1. Summary of experimental multiplicity results................................................. 36

Table 12.2. Comparison of multiplicity rates from a JSR14 and the VME system................... 39

Table 13.1. Geant4 model results from multiplicity analysis for various materials................... 43

Table 13.2. Model results for total outgoing neutrons for materials for $10^{5}$ incoming neutrons.. 44

Table 13.2. Ratio of outgoing neutrons for $\mathrm{Cu} / \mathrm{Pb}$ for three spectra...................................... 46

Table 14.1. Ratio of outgoing neutrons to incoming neutrons for $\mathrm{Pb}$ cube .............................. 48

Table 14.2. MCNP results percent of unscattered incident neutrons..................................... 51

Table 14.3. MCNP results for total neutrons out for cosmic ray components on materials........ 54

Table 14.4. MCNP results for total neutrons out for cosmic ray components on materials........ 56

Table 15.1. Data for various materials shown in Figure 15-1.............................................. 59 


\section{Introduction}

Cosmic ray primaries create showers in the atmosphere that include a broad spectrum of neutrons, protons and muons. At the Earth's surface, the muon flux (168 $\mathrm{m}^{-2} \mathrm{~s}^{-1}$ [Greisen 1942]) and neutron flux (134 $\mathrm{m}^{-2} \mathrm{~s}^{-1}$ [Gordon 2004]) are comparable, while the proton flux $\left(2 \mathrm{~m}^{-2} \mathrm{~s}^{-1}\right.$ [Diggory 1974; Grieder 2001; Nakamura 2012]) is a few percent of these. These neutrons, muons and protons interact with materials at the surface of the Earth, yielding prompt backgrounds in radiation detection systems, as well as inducing long-lived activation products through spallation events. Spallation events involve a cascade of neutron interactions in a block of material following knock-out interaction that "breaks" a nucleus apart. Muon and proton induced spallation are about an order of magnitude smaller than neutron induced events at the surface of the Earth. The multiple neutrons produced in spallation events are referred to as "ship effect" neutrons. ${ }^{1}$ Quantifying the background from cosmic ray induced activities is important to low-background experiments, such as neutrinoless double-beta decay (0v $\beta \beta)$ [Aalseth 2009].

\subsection{Motivation for This Study}

The experimental measurements reported here were initiated to try to validate results from Geant4 Monte Carlo models to provide some confidence in the modeling results. The results have been used, for example, to predict the size of shielding needed on the surface of the Earth for reducing exposure of transported materials. Such predictions require a high degree of confidence in the simulations.

The European ${ }^{76} \mathrm{Ge}$ neutrinoless double-beta decay experiment (GERDA) has used Monte-Carlo simulations to design a transport shield, using ISABEL data and the SHIELD code [Barabanov 2006]. Figure 1-1 shows a cutaway view of this shield design, which is $126.5 \mathrm{~cm}$ high by 140 $\mathrm{cm}$ in diameter.

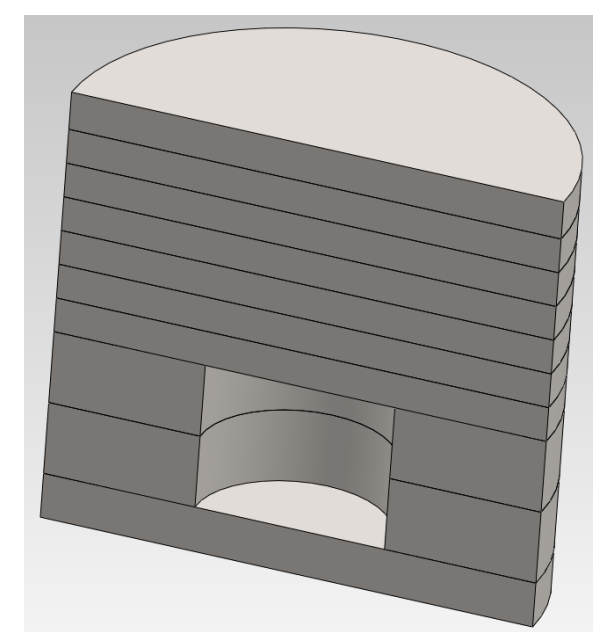

Figure 1-1. Cutaway view of GERDA shield design

\footnotetext{
${ }^{1}$ The term "ship effect" arises from the observation of the increased neutron flux from spallation near large ships that was observed when U.S. detectors looking for nuclear weapons were used near Soviet ships.
} 
The SHIELD result predicts an attenuation length for neutrons at $100 \mathrm{MeV}$ in iron of about 240 $\mathrm{g} / \mathrm{cm}^{2}(0.30 \mathrm{~m})$. Barabanov et al. predict that the shield design of Figure 1-1 will reduce production of ${ }^{68} \mathrm{Ge}$ and ${ }^{60} \mathrm{Co}$ by 10 and 15 , respectively. They also state that calculations using the library of excitation functions, ISABEL, gave results that were a factor of 2 to 6 lower (i.e., less production of ${ }^{68} \mathrm{Ge}$ and ${ }^{60} \mathrm{Co}$ due to more attenuation of neutrons predicted by ISABEL) than those obtained with their simulation tool, SHIELD.

The Geant4 model results performed for the MAJORANA DEMONSTRATOR for this same transport shield disagreed with the Barabanov simulations using SHIELD by a factor of $\sim 2.5$ for the effect of iron as a shield material [Aguayo 2012b]. Geant 4 predicted a greater degree of shielding by the iron shipping shield than the SHIELD code. This difference is what motivated the current study to see how well a model could predict experimental results.

When energetic neutrons interact with nuclei in a material, they can cause spallation of the nucleus, releasing a large number of neutrons that then cascade through the material. A high efficiency thermal-neutron detector surrounding a block of material can detect some fraction of these spallation neutrons. The number of neutrons detected in a specified time window is referred to here as the "multiplicity." Recording the timing of each detected neutron can be used to generate a histogram of the measured multiplicity. For long enough time scales, much greater than the $\sim 0.1$ millisecond thermalization time for neutrons from a spallation event, the distribution will look random (Poisson). For short time scales comparable to the neutron thermalization time (less than $\sim 1$ millisecond), high multiplicity events from the ship effect will show up in the multiplicity as a non-Poison distribution. One measure of this non-Poisson behavior is to count the number of events that exceed some multiplicity value (e.g., four or more), and such measures are used here to show the dependence of multiplicity on material type. It has been demonstrated previously that there is a strong dependence of multiplicity on material atomic mass; the current work provides a more controlled measurement than was previously reported [Kouzes 2008].

This paper reports on neutron multiplicity measurements from cosmic neutron interactions in different materials for the purpose of comparing them to predictions from Monte Carlo models. Neutron multiplicity was measured, and modeled, for commonly used shielding materials (polyethylene, aluminum, steel, copper, lead and tungsten), and the results are compared.

\subsection{Cosmic Ray Neutron Spectra}

Since direct measurements of the effects of shielding on the cosmic ray neutron spectrum are not available, Monte Carlo modeling with Geant4 [Agostinelli 2003] has been used to compute such effects. However, there are large uncertainties (orders of magnitude) in the cosmic ray neutron spectrum and the possible cross-section libraries used for such calculations.

Figure 1-2 shows cosmic ray neutron energy spectra from $1 \mathrm{MeV}$ to $1 \mathrm{GeV}$ as measured by various authors [Armstrong 1973; Gordon 2004; Hess 1959; Ziegler 1998] and computed by the CRY model [Hagmann 2008]. The energy region from about $20 \mathrm{MeV}$ to $200 \mathrm{MeV}$ is of most interest to production of backgrounds in materials used for $0 v \beta \beta$. The cosmic spectrum approximately follows an $\mathrm{E}^{-1}$ dependence, as shown by the dotted line. The CRY code results do not appear to be consistent with the experimental values for these low energies of interest. The CRY results are not used because of this discrepancy. 


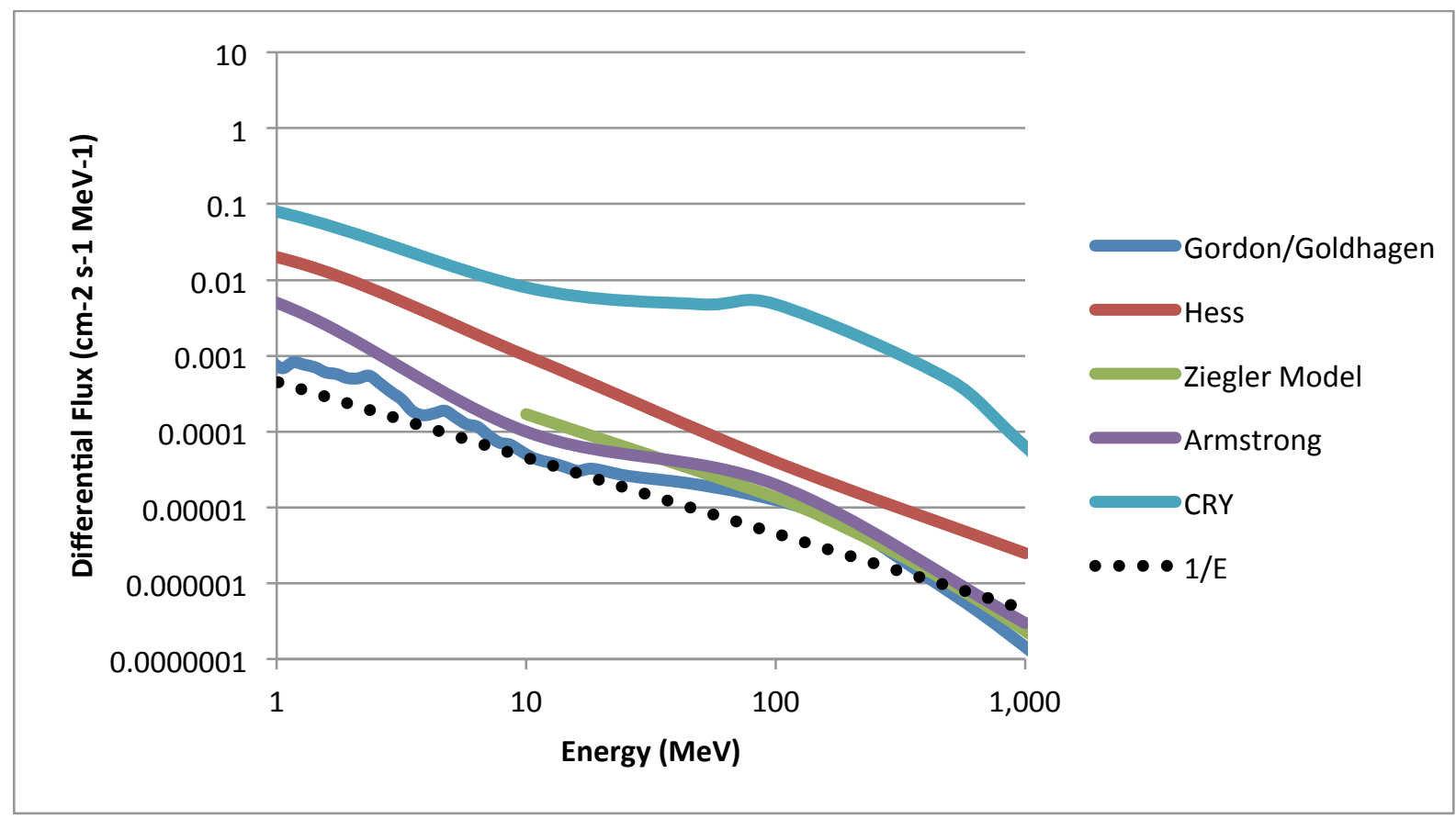

Figure 1-2. Cosmic ray neutron energy spectra from $1 \mathrm{MeV}$ to $1 \mathrm{GeV}$ from various authors.

\subsection{Neutron Cross-Sections}

The plots in Figure 1-3 show an example of the large variation in reaction cross-section libraries that can be selected in Geant4 for production of one of the radionuclides of interest for $0 v \beta \beta$ $\left({ }^{68} \mathrm{Ge}\right)$. These order of magnitude uncertainties directly impact the predictive ability of Geant 4 for cosmogenic production of backgrounds in $0 v \beta \beta$ experiments. The G4NDL4.0 cross-sections have been used for Geant 4 calculations [Aguayo 2012b]. The ISABEL libraries were used for shield calculations [Barabanov 2006]. 


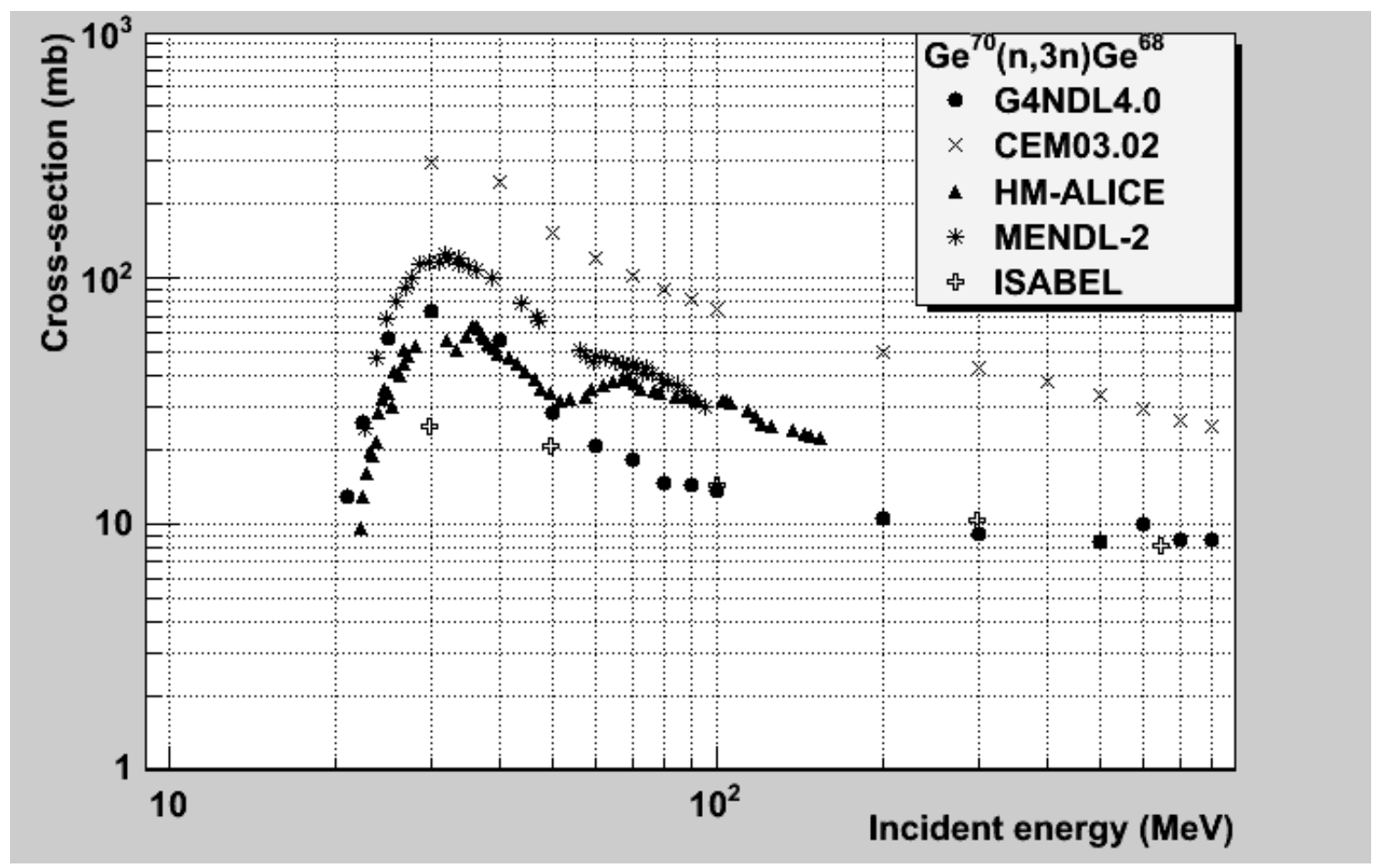

Figure 1-3. Differential neutron cross-section example.

Figures 1-4 through 1-9 show the total cross-sections used in Geant4 for neutron reactions on the various materials of interest for this study. Only neutrons above about $20 \mathrm{MeV}$ are of sufficient energy to produce radionuclides that can be backgrounds in $0 v \beta \beta$ experiments. Note that the "capture" process ends abruptly at tens of $\mathrm{MeV}$, indicating some limitation of the models, though it is a small contribution above this energy. 


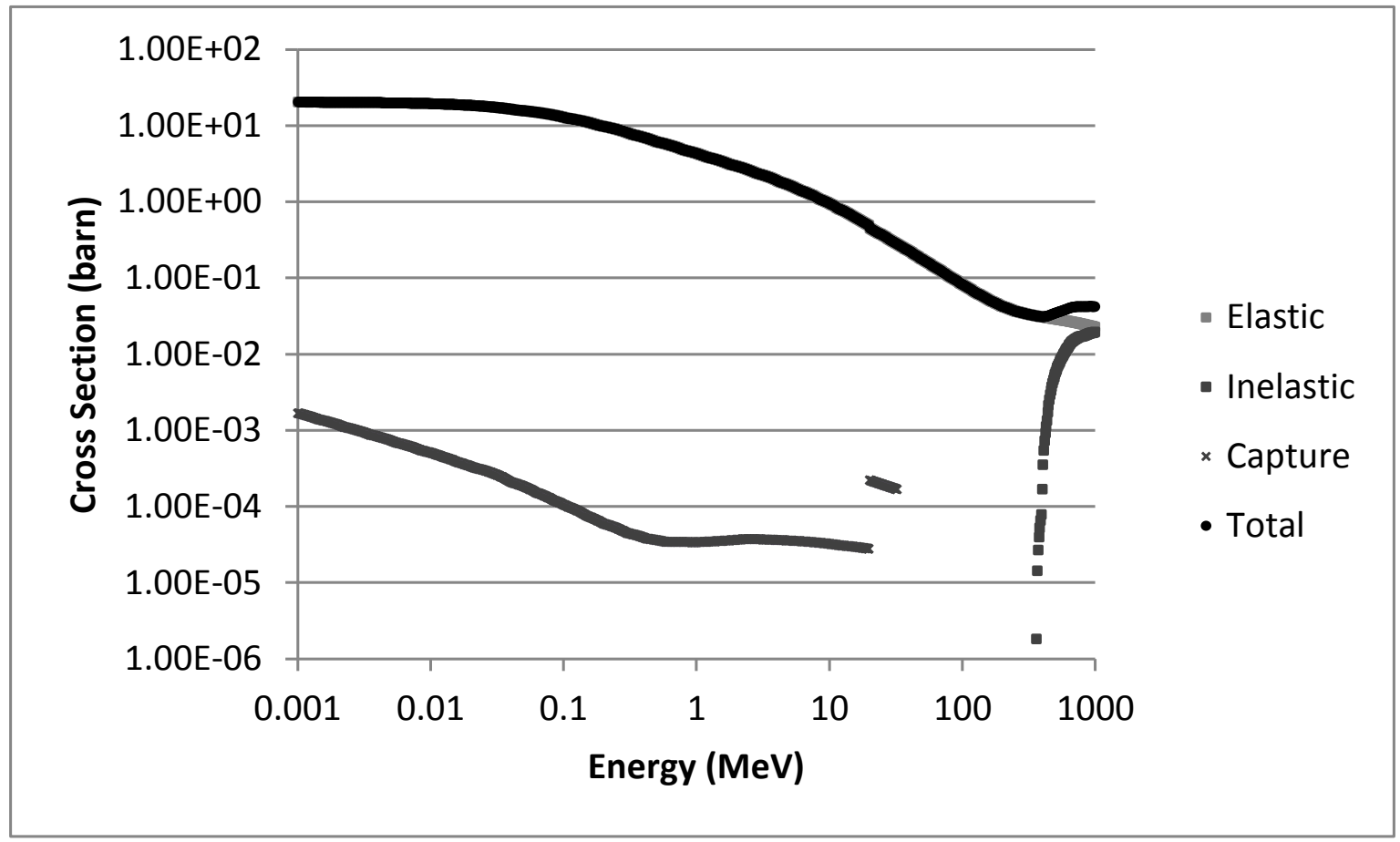

Figure 1-4. Total cross section for neutron processes in polyethylene used in Geant4

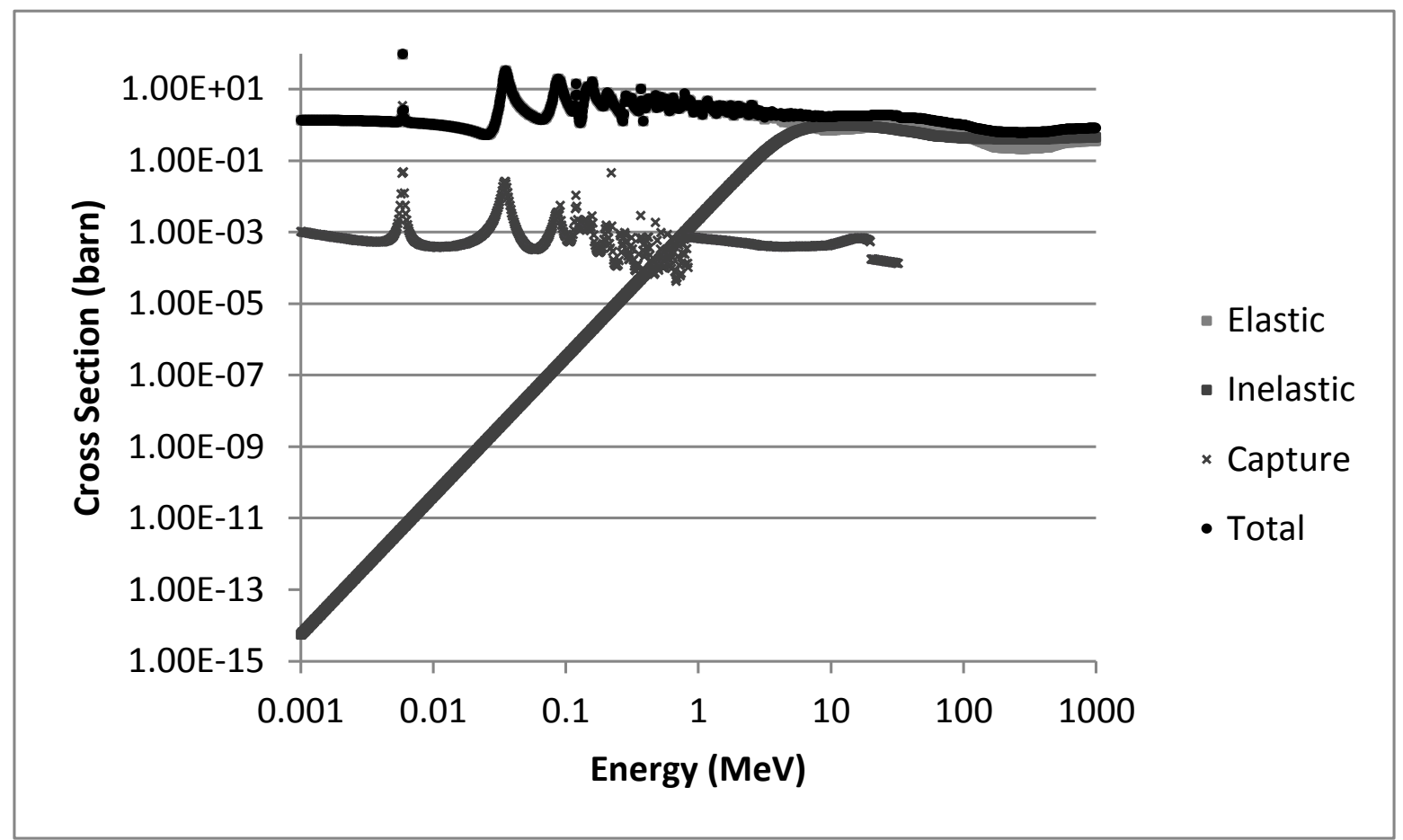

Figure 1-5. Total cross section for neutron processes in Al used in Geant4 


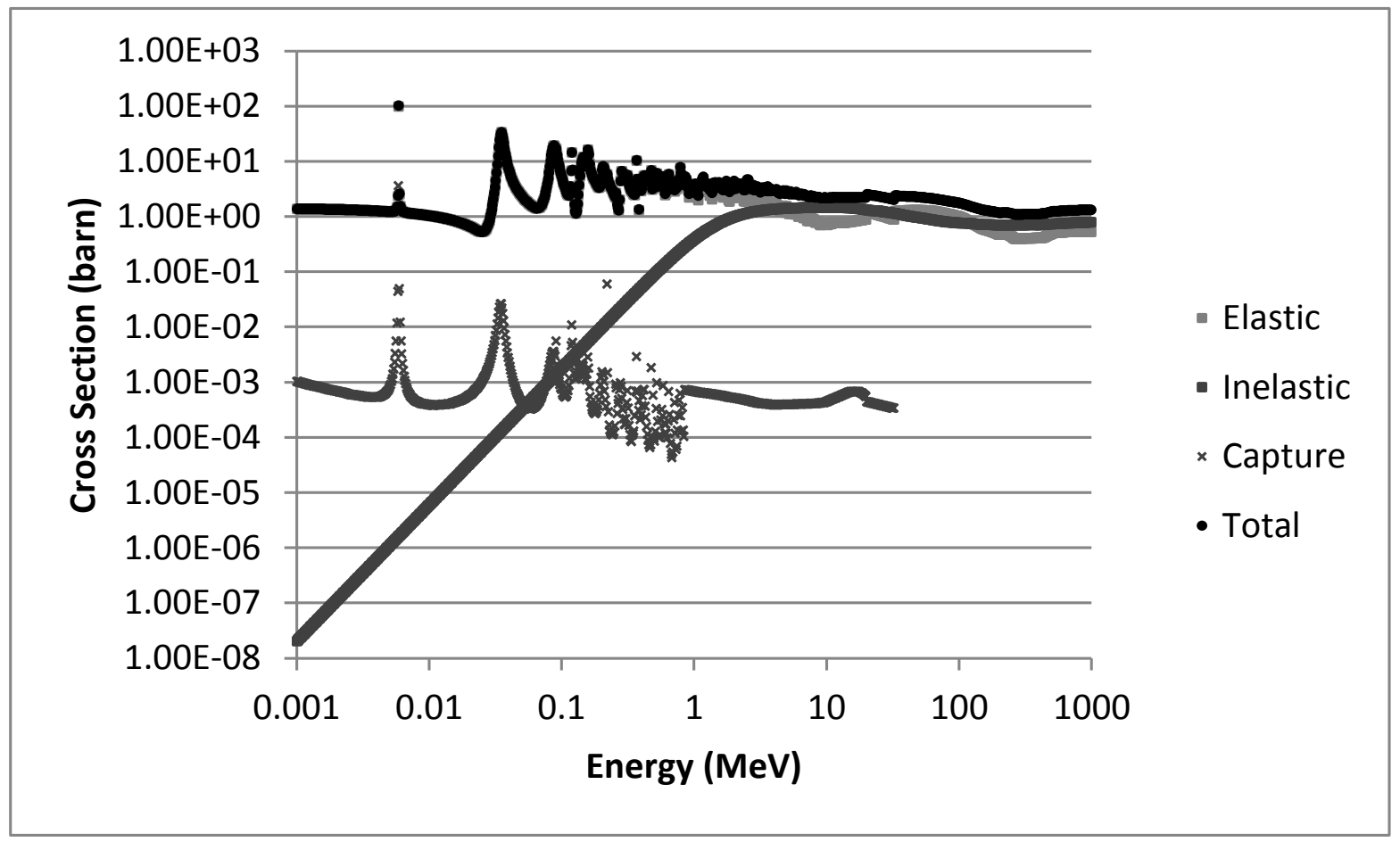

Figure 1-6. Total cross section for neutron processes in Fe used in Geant4

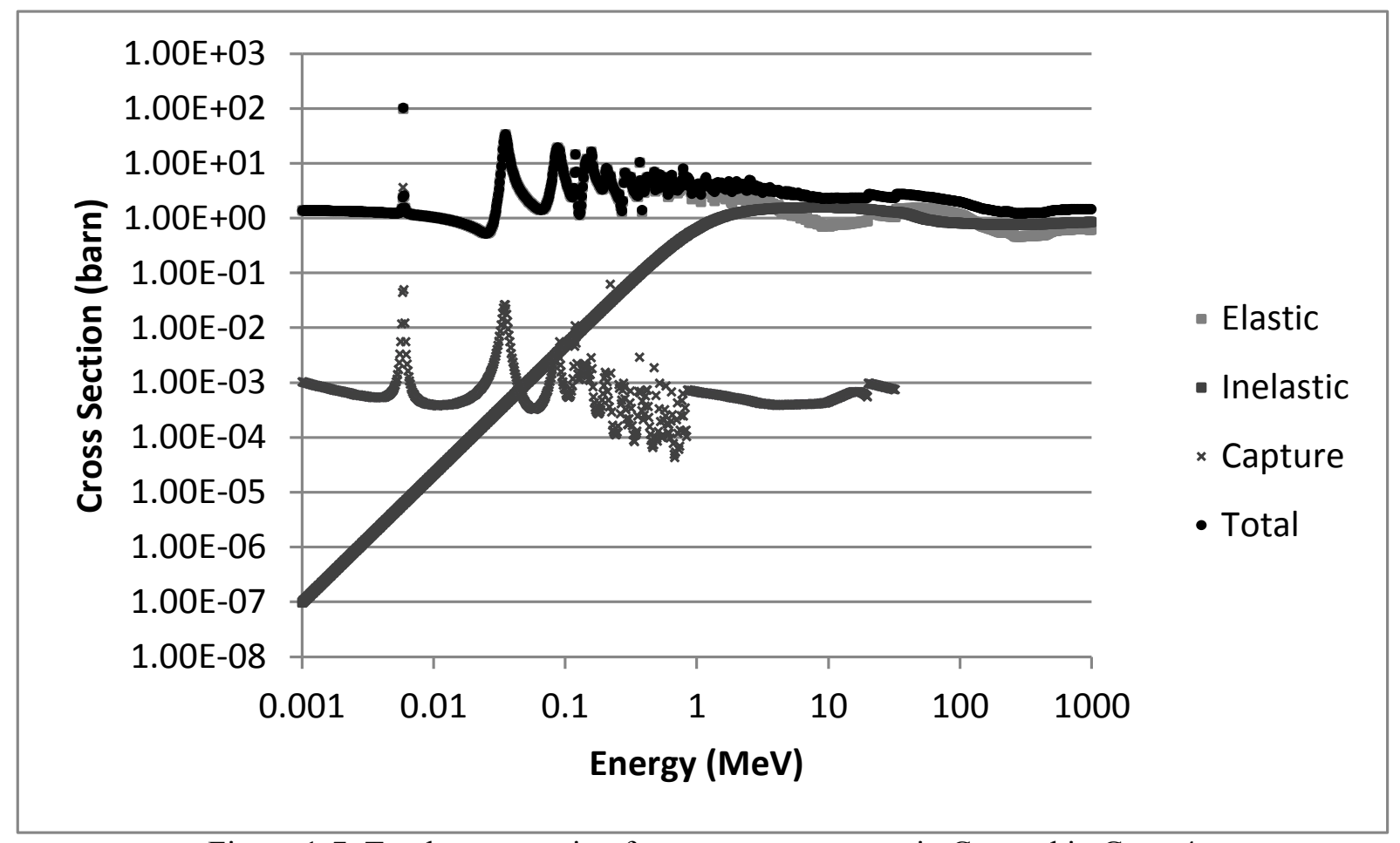

Figure 1-7. Total cross section for neutron processes in $\mathrm{Cu}$ used in Geant4 


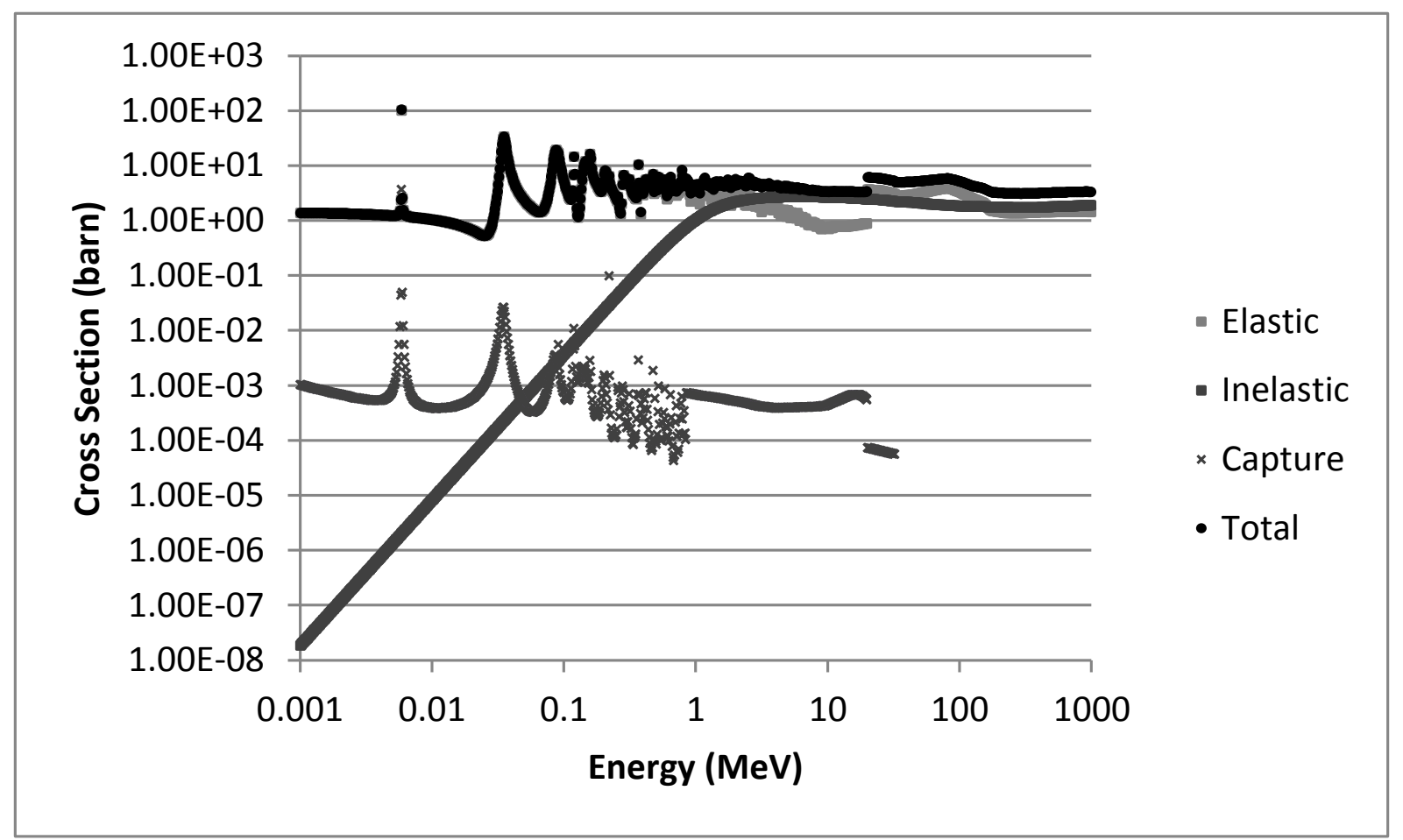

Figure 1-8. Total cross section for neutron processes in $\mathrm{Pb}$ used in Geant4

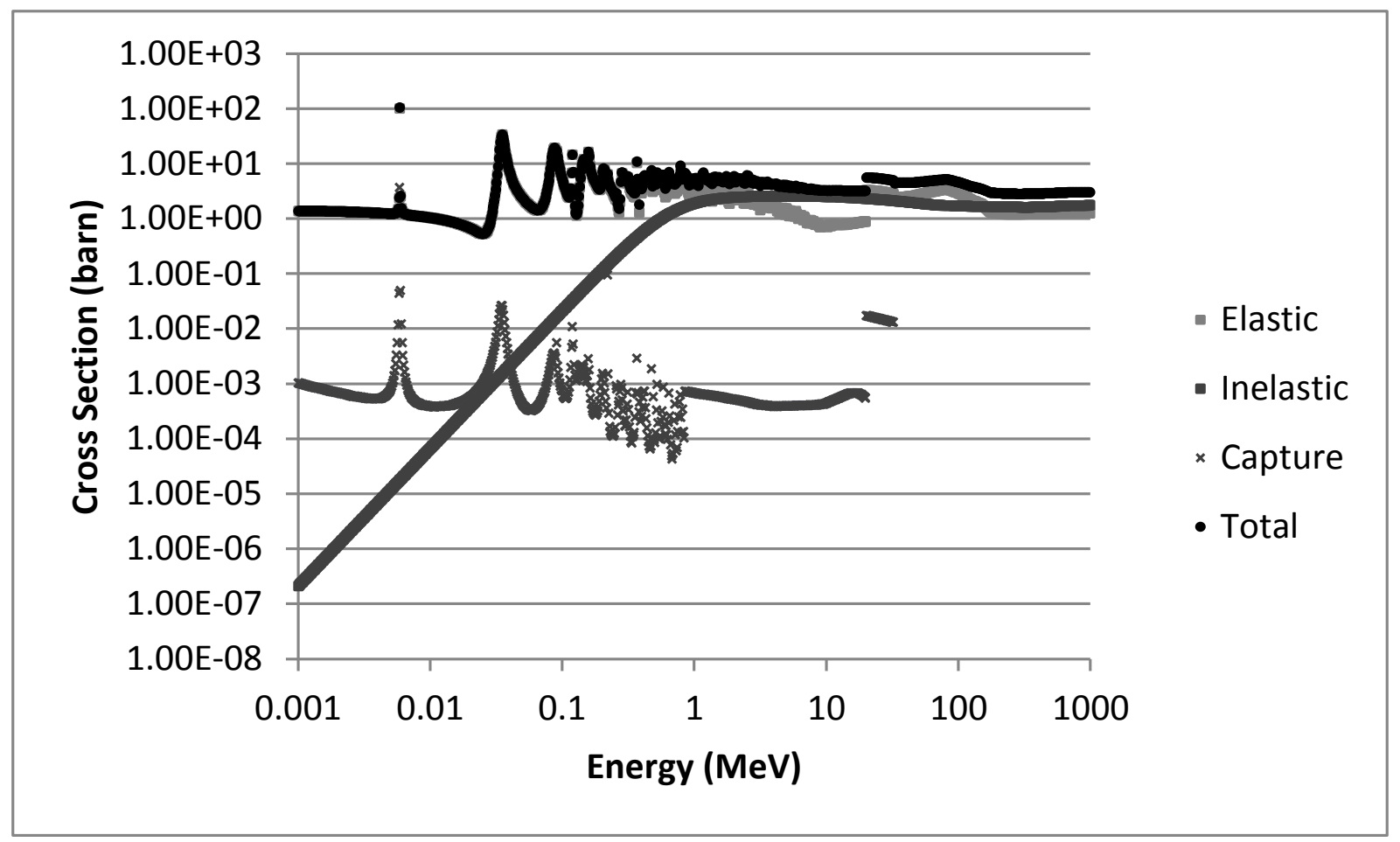

Figure 1-9. Total cross section for neutron processes in $\mathrm{W}$ used in Geant4 


\subsection{Samples Used}

Table 1.1 lists the mass and volume of samples of each material, and the mass and volume used as the total target in the measurements. The measured mass and measured volume are for the samples of material that were actually weighed, and the target mass and volume are for the complete sample used in the multiplicity measurements. The published densities listed were used for this study since the measurements of the material sizes were approximate. This introduces approximately a $10 \%$ uncertainty in the assumed material densities. The neutron density listed is the number of neutrons per cubic centimeter times $10^{-24}$.

The approach to the measurements was to use equal volumes and geometries for all materials measured in order to eliminate corrections for geometry. This was possible for all measurements except for tungsten, where a smaller volume was used due to the expense of obtaining a full volume of that material.

Table 1.1. Sample masses and volumes used in the runs.

\begin{tabular}{|l|l|l|l|l|l|l|}
\hline Material & $\begin{array}{l}\text { Density } \\
\text { (g/cc) }\end{array}$ & $\begin{array}{l}\text { Neutron } \\
\text { Density } \\
(\mathbf{n} / \mathbf{c c}) \mathbf{x 1 0}\end{array}$ & $\begin{array}{l}\text { Measured } \\
\text { mass (kg) }\end{array}$ & $\begin{array}{l}\text { Measured } \\
\text { volume (l) }\end{array}$ & $\begin{array}{l}\text { Target mass } \\
\text { (kg) }\end{array}$ & $\begin{array}{l}\text { Target } \\
\text { volume } \\
\text { (l) }\end{array}$ \\
\hline $\mathrm{HDPE}$ & 0.95 & 0.25 & 4.4 & 4.7 & 28.05 & 28.4 \\
\hline $\mathrm{Al}$ & 2.7 & 0.84 & 4.8 & 1.8 & 76.8 & 28.4 \\
\hline $\mathrm{Fe}$ & 7.87 & 2.56 & 37.6 & 4.8 & 222.6 & 28.4 \\
\hline $\mathrm{Cu}$ & 8.94 & 3.22 & 48.5 & 4.9 & 237.9 & 28.4 \\
\hline $\mathrm{Pb}$ & 11.3 & 4.12 & 53.3 & 4.7 & 320.1 & 28.4 \\
\hline $\mathrm{W}$ & 19.3 & 6.52 & 52.9 & 3 & 52.89 & 3 \\
\hline
\end{tabular}

\subsection{Measurements}

When a cosmic neutron interacts with the target material and produces a spallation event, a small fraction of the multiple neutrons produced are thermalized and detected by the multiplicity counter (described in the next Section). The total-neutron (infinite time gate) detection efficiency of the detector used for the neutrons measurements reported here (thermalization and detection) was measured to be approximately $17 \%$ using ${ }^{252} \mathrm{Cf}$, as will be discussed. The energies of the neutrons produced in a spallation event tend to be in the few $\mathrm{MeV}$ range, and it was assumed that this distribution is independent of material type. It was further assumed that neutrons have an equal probability of leaving the volume of the material and being detected, independent of the material type. This is not an unreasonable assumption since the fast neutron cross-sections for these materials are comparable. But, these assumptions require testing through model studies, as will be discussed.

All measurements were made at the 3440 Building at Pacific Northwest National Laboratory (PNNL). Table 1.2 lists the measurements made as part of this study. The muon flux was measured with the $\mu \mathrm{Witness}$ counter as discussed in a later Section. 
Table 1.2. Measurements made as part of this study.

\begin{tabular}{|c|c|c|c|c|c|c|}
\hline Date & Target material & $\begin{array}{l}\text { Events } \\
\text { recorded }\end{array}$ & $\begin{array}{c}\text { Run } \\
\text { time (s) }\end{array}$ & $\begin{array}{l}\text { Dead } \\
\text { time }\end{array}$ & $\begin{array}{c}\text { Singles } \\
\text { Count Rate } \\
\text { (neutrons/s) }\end{array}$ & $\begin{array}{l}\text { Average muon } \\
\text { rate during run } \\
(\mu \text { Witness })(\mu / s)\end{array}$ \\
\hline $12 / 7 / 2012$ & Poly-Ethylene & 600000 & 64708 & $1.4 \%$ & $9.3 \pm 1.5$ & N/A \\
\hline $12 / 11 / 2012$ & Steel & 600000 & 61105 & $1.4 \%$ & $9.8 \pm 1.5$ & N/A \\
\hline $12 / 10 / 2012$ & Air (Background) & 600000 & 63078 & $1.4 \%$ & $9.5 \pm 1.5$ & N/A \\
\hline $12 / 12 / 2012$ & Copper & 600000 & 53748 & $1.4 \%$ & $11.3 \pm 1.5$ & 2.23 \\
\hline $12 / 13 / 2012$ & Copper & 600000 & 54936 & $1.4 \%$ & $11.1 \pm 1.5$ & 2.24 \\
\hline $12 / 14 / 2012$ & Copper & 600000 & 52651 & $1.5 \%$ & $11.6 \pm 1.5$ & 2.26 \\
\hline $12 / 18 / 2012$ & Copper & 600000 & 53452 & $1.5 \%$ & $11.4 \pm 1.5$ & 2.26 \\
\hline $1 / 3 / 2013$ & Copper & 600000 & 55592 & $1.4 \%$ & $11.0 \pm 1.5$ & 2.32 \\
\hline $1 / 6 / 2013$ & Air (Background) & 600000 & 58680 & $1.4 \%$ & $10.3 \pm 1.5$ & 2.28 \\
\hline $1 / 07 / 2013$ & Lead & 600000 & 42125 & $1.6 \%$ & $14.5 \pm 1.5$ & 2.31 \\
\hline $1 / 08 / 2013$ & Lead & 600000 & 42844 & $1.6 \%$ & $14.2 \pm 1.5$ & 2.32 \\
\hline $1 / 10 / 2013$ & Air (Background) & 600000 & 62786 & $1.4 \%$ & $9.7 \pm 1.5$ & 2.35 \\
\hline $1 / 11 / 2013$ & Steel & 600000 & 60780 & $1.4 \%$ & $10.0 \pm 1.5$ & 2.34 \\
\hline $1 / 13 / 2013$ & Air (Background) & 600000 & 65745 & $1.4 \%$ & $9.4 \pm 1.5$ & 2.35 \\
\hline $1 / 14 / 2013$ & Poly-Ethylene & 600000 & 71321 & $1.4 \%$ & $8.5 \pm 1.5$ & 2.24 \\
\hline $1 / 15 / 2013$ & Air (Background) & 600000 & 65241 & $1.4 \%$ & $9.3 \pm 1.5$ & 2.26 \\
\hline $1 / 17 / 2013$ & Tungsten & 600000 & 65557 & $1.5 \%$ & $9.3 \pm 1.5$ & 2.24 \\
\hline $1 / 18 / 2013$ & Air (Background) & 600000 & 63576 & $1.4 \%$ & $9.6 \pm 1.5$ & 2.23 \\
\hline $1 / 20 / 2013$ & Poly-Ethylene & 600000 & 70003 & $1.4 \%$ & $8.6 \pm 1.5$ & 2.19 \\
\hline $3 / 1 / 2013$ & Aluminum & 600000 & 70985 & $1.4 \%$ & $8.5 \pm 1.5$ & $\mathrm{~N} / \mathrm{A}$ \\
\hline $7 / 23 / 2013$ & Air (Background) & 600000 & 57763 & $1.2 \%$ & $10.4 \pm 1.5$ & $\mathrm{~N} / \mathrm{A}$ \\
\hline $7 / 24 / 2013$ & Lead $(4 \times 4 \times 4$ in. $)$ & 400000 & 35550 & $1.2 \%$ & $11.3 \pm 1.5$ & $\mathrm{~N} / \mathrm{A}$ \\
\hline $8 / 4 / 2013$ & Lead $(8 \times 8 \times 4$ in. $)$ & 400000 & 28167 & $1.4 \%$ & $14.2 \pm 1.5$ & $\mathrm{~N} / \mathrm{A}$ \\
\hline $8 / 6 / 2013$ & Lead (8x8x8 in.) & 400000 & 26266 & $1.2 \%$ & $15.2 \pm 1.5$ & $\mathrm{~N} / \mathrm{A}$ \\
\hline $8 / 9 / 2013$ & Air (Background) & 600000 & 43292 & $1.4 \%$ & $13.9 \pm 1.5$ & $\mathrm{~N} / \mathrm{A}$ \\
\hline $8 / 13 / 2013$ & Lead (12x8x8 in.) & 400000 & 25068 & $1.3 \%$ & $16.0 \pm 1.5$ & $\mathrm{~N} / \mathrm{A}$ \\
\hline $8 / 14 / 2013$ & Lead (12x12x8 in.) & 400000 & 23180 & $1.2 \%$ & $17.3 \pm 1.5$ & N/A \\
\hline $8 / 16 / 2013$ & Lead (12x12x12 in.) & 400000 & 25589 & $1.2 \%$ & $15.6 \pm 1.5$ & N/A \\
\hline $8 / 28 / 2013$ & 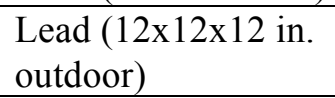 & 400000 & 29840 & $1.2 \%$ & $13.5 \pm 1.5$ & N/A \\
\hline
\end{tabular}




\section{Multiplicity Counter System}

A neutron multiplicity counter using an array of ${ }^{3} \mathrm{He}$ tubes in a polyethylene moderator was used for the measurements presented in this report [Switzer 2003]. The detector was outfitted with a custom data acquisition system to log neutron capture events. The assembly was installed on a rolling cart, as seen in Figure 2-1, so it could be transported to a measurement location with a thin roof so as not to significantly impact the cosmic ray neutrons. From the acquired data, multiplicity was extracted using various coincidence resolving windows $(100 \mathrm{~ms}, 10 \mathrm{~ms}, 1 \mathrm{~ms}$ and $100 \mu \mathrm{s})$. The neutron multiplicity counter used was an array of 12 commercially available ${ }^{3} \mathrm{He}$-filled gas proportional counters from GE Reuter Stokes (Twinsburg, OH). The proportional tubes ( $0.95 \mathrm{~m}$ long, $0.05 \mathrm{~m}$ outer diameter, $4 \mathrm{~atm}$.) were configured in four sets of three tubes, with each set enclosed in polyethylene moderator in an aluminum box, as can be seen in the top view shown in Figure 2-2.

The four sets of tubes were configured to form a box with an inner cavity opening of $30.5 \mathrm{~cm}$ square ( 1 square foot), in a well detector fashion. The target materials were placed in the inner cavity on top of a $35.6 \mathrm{~cm}$ high wooden support so that they were in the vertical center of the detector array. The 12 proportional counters were operated at $1200 \mathrm{~V}$ and were connected to three preamplifiers with their outputs OR-ed together to produce a single transistor-transistor logic (TTL) output signal. This TTL signal was fed into a Versa Module Eurocard (VME) based analog to digital converter (ADC) manufactured by Struck Innovative Systeme (Hamburg, Germany) that generated a time stamp for each pulse. A graphical interface capable of plotting multiplicity events within a user defined time window was developed for analysis. 


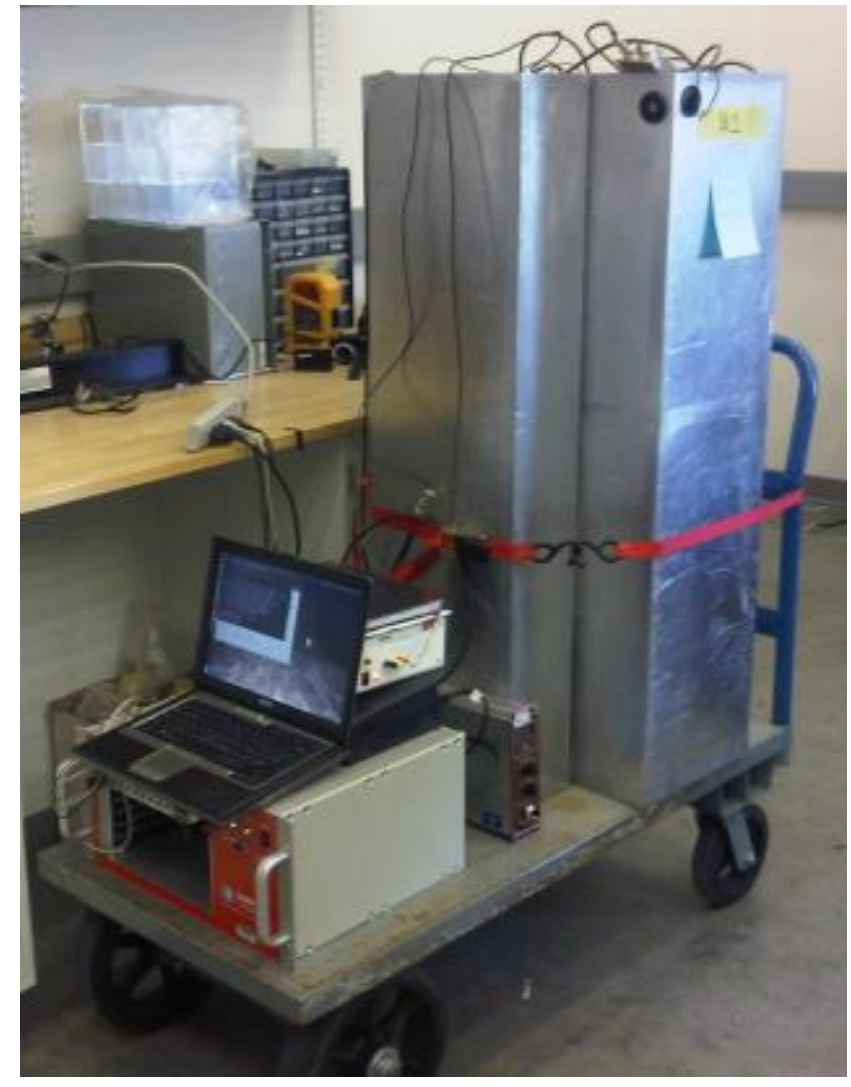

Figure 2-1. Experimental set-up of the multiplicity counter and electronics at the 3440 building.

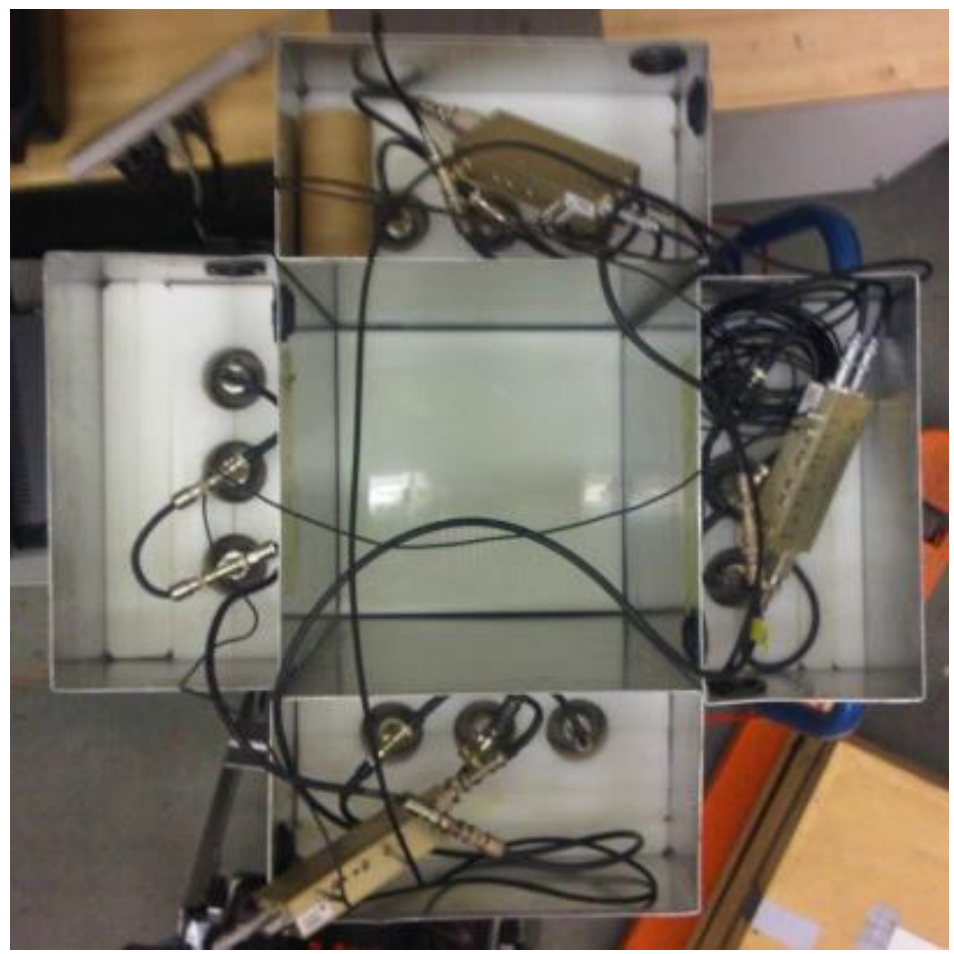

Figure 2-2. Top view of the multiplicity counter (with polyethylene sample). 
The fast ADC allowed collecting multiplicity distribution information in a range from one to 80 counts in the gate width. The system was designed to minimize electronic dead time between counts using a small but fast access memory where the data is logged for up to 80 consecutive counts, then the content of this first memory is transferred to the personal computer (PC). The dead time for a memory access in the first data tier is $150 \mathrm{~ns}$, whereas the dead time for the Tier 1 memory transfer to the PC is $10 \mathrm{~ms}$. Having these set dead times for memory access allows for a simple calculation to determine the effective dead time of the system. The digitization frequency of this ADC is $100 \mathrm{MHz}$, with a time stamp option for every digitized pulse. This feature was used to give the system the ability to count multiplicities in a user defined gate width with multiplicity up to 80 . Since the event rate was of order $10 \mathrm{~Hz}$, dead time for the measurements was minimal.

The operation of the multiplicity counter was in two steps. The first step was to log every incoming pulse, along with the time stamp, into a memory buffer. The memory buffer fills up with the pre-programmed number of events to be recoded. The multiplicity counter was programmed to log a fixed number of total counts, rather than a set run time.

The second step was to apply offline analysis to this data to extract the multiplicity distribution based on the time stamps of the recorded pulses. The multiplicity distribution can be determined by generating a histogram of the number of events with a given number of pulses following in a fixed time window. In order to measure multiplicity, the algorithm looked in a time window starting with the first pulse and counted the number of pulses. For example, assume a pulse comes in at $10 \mu \mathrm{s}$ after the counter starts operating. The next pulses come in at times $10.2 \mu \mathrm{s}$ and $12 \mu \mathrm{s}$, and no pulses after a $2 \mu \mathrm{s}$ window. If the programmed gate length was larger than $0.2 \mu \mathrm{s}$ and smaller than $2 \mu \mathrm{s}$, multiplicity bin 2 would be incremented by one unit, and the multiplicity bin 1 would also be incremented by one unit. If the programmed gate width were larger than 2 $\mu \mathrm{s}$, the multiplicity bin 3 would be incremented by one. If the gate width were shorter than 0.2 us, multiplicity bin 1 would be incremented three times. This analysis algorithm prevents double counting of pulses in different multiplicity bins. This rationale is valid for multiplicity counting in a scenario where the incoming pulse rate is below that of the inverse of the gate length time. This is required in order to be able to ignore situations were the pulse train might induce miscounting due to event pileup from different primary nuclear reactions. This is the case for cosmogenic neutron counting, where a simplistic analysis was possible since the event rate was low, making the traditional coincidence analysis of reals and accidentals unnecessary.

Post analysis of the data involved determining multiplicity with time windows of $100 \mathrm{~ms}, 10 \mathrm{~ms}$, $1 \mathrm{~ms}$ and $100 \mu \mathrm{s}$. Typical die-away times for coincidence counters are in the 50-100 $\mathrm{ss}$ regime, so a $100 \mu$ s time window is perhaps appropriate, while $100 \mathrm{~ms}$ window is too long, allowing the multiplicity distribution to be averaged out. Since the flux of cosmic neutrons is about $134 \mathrm{~m}^{-2} \mathrm{~s}^{-1}$ [Gordon 2004], the average time between spallation events should be fewer than about one every $80 \mathrm{~ms}$ since not every neutron will produce a spallation. A $1 \mathrm{~ms}$ time window is reasonable for data analysis, long enough to capture the full multiplicity of events but short enough not to be contaminated by overlapping events.

An MCNP model [MCNP] of the multiplicity counter had previously been developed [Switzer 2003]. Side and top views of the model developed in that study are shown in Figure 2-3. 

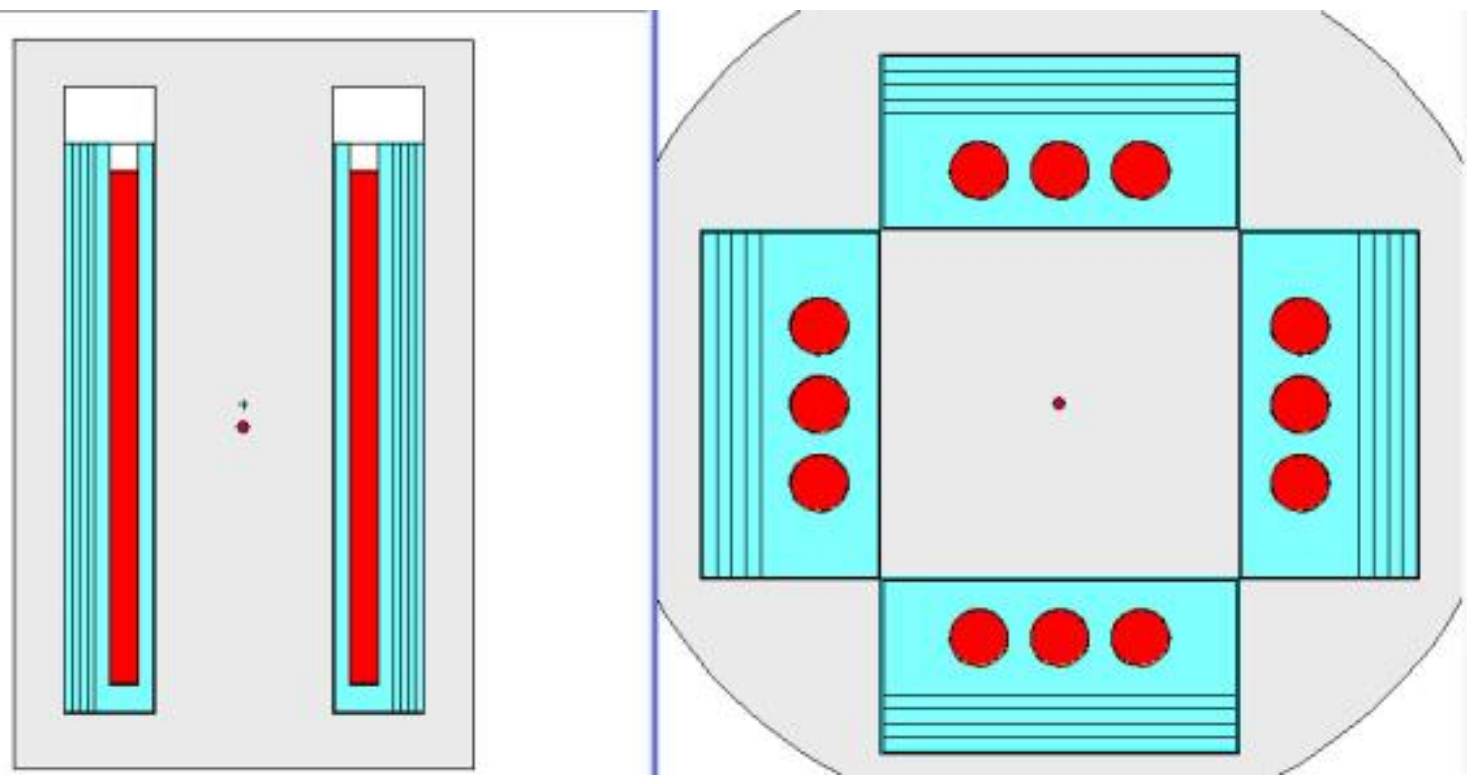

Figure 2-3. Side and top view of the multiplicity counter in the MCNP model.

The MCNP model predicts an efficiency of $19.9 \%$ for a centered ${ }^{252} \mathrm{Cf}$ source, and a die-away time of $46 \mu \mathrm{s}$. Thus, a time window of $60-100 \mu \mathrm{s}$ is reasonable for counting coincidences from spallation events (which occur on a much shorter time scale). Energy and (vertical) position efficiency profiles were also calculated, as shown in Figure 2-4. The energy profile shows a reasonably flat behavior for spallation neutrons with energies between $\sim 100 \mathrm{eV}$ to $100 \mathrm{keV}$, a gradual fall off below $100 \mathrm{eV}$, but a rapid fall off above $100 \mathrm{keV}$. The position profile shows there can be $\sim 10 \%$ reduction in efficiency if neutrons are emitted from the spallation block at positions $\sim 20 \mathrm{~cm}$ above or below the center of the detector. These effects are automatically taken into account for MCNP calculations for finite blocks of material. 


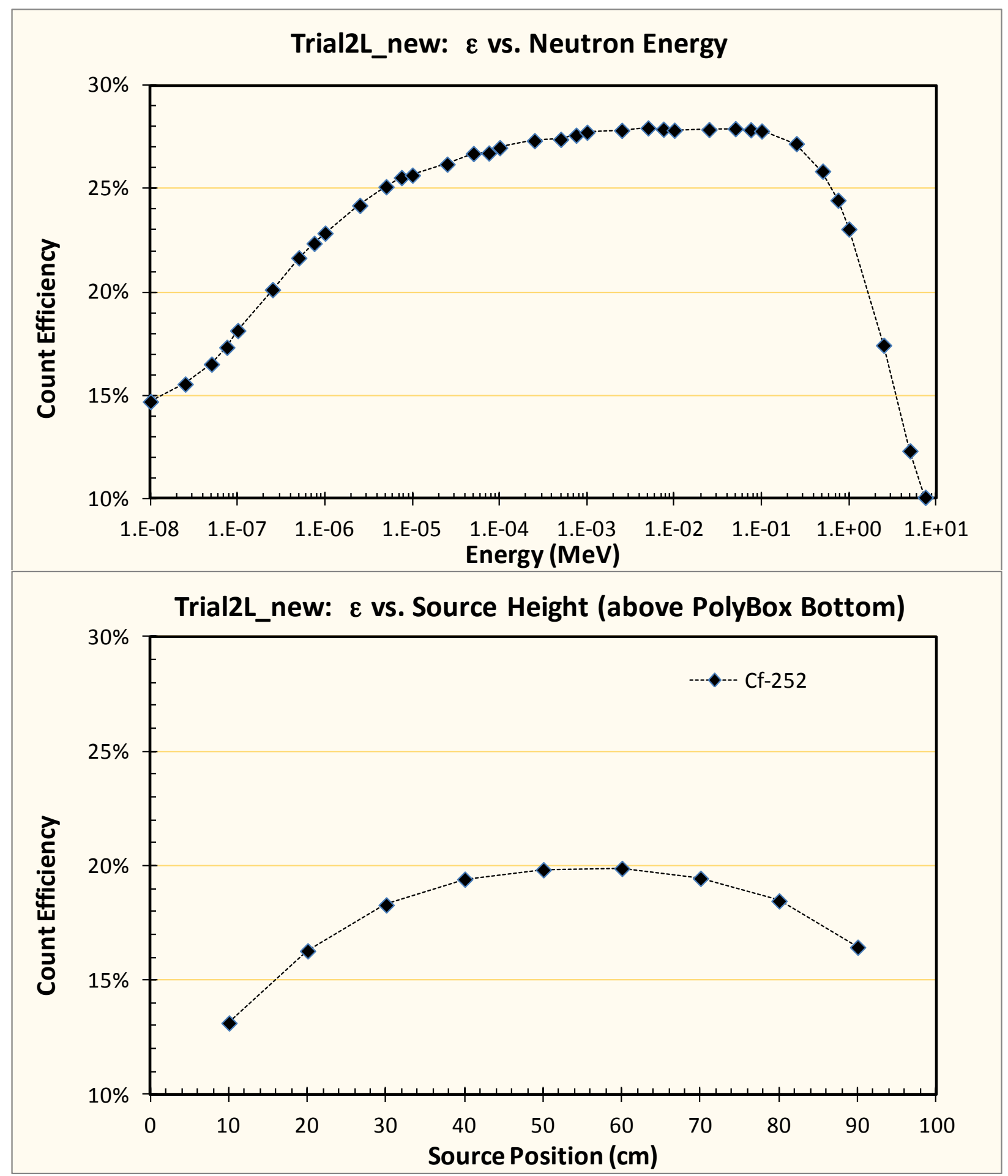

Figure 2-4. Energy and position efficiency profiles for 16-tube coincidence counter used. 


\section{Neutron Efficiency and Gamma Ray Sensitivity Measurements}

A gamma ray source was used to verify that the multiplicity detector threshold was adjusted appropriately to be insensitive to gamma rays. This was done by placing ${ }^{137} \mathrm{Cs}(7.7 \mu \mathrm{Ci})$ and ${ }^{60} \mathrm{Co}$ $(2.7 \mu \mathrm{Ci})$ sources in the center of the detector and finding that there was no change in the neutron background count rate observed by the detector system.

The absolute efficiency $(\varepsilon)$ of the multiplicity detector was measured using a bare ${ }^{252} \mathrm{Cf}$ fission neutron source placed in the center of the detector system. The efficiency was measured twice. The first measurement performed used the VME electronics, which have a significant dead time for the data rate obtained with the source. Details of the VME run are given in Table 3.1.

Table 3.1. Source run parameters from VME system (November 28, 2012)

\begin{tabular}{|l|l|}
\hline \multicolumn{1}{|c|}{ Parameter } & \multicolumn{1}{c|}{ Value } \\
\hline Events recorded & 85780 \\
\hline Run time & $130.8 \mathrm{~s}$ \\
\hline Dead time & $71.9 \mathrm{~s}(54.9 \%)$ \\
\hline Average time between pulses & $702 \mu \mathrm{s}(1424 \mathrm{cps})$ \\
\hline Source Activity $(\# 56595-130 \mathrm{E})$ & $1.9 \mu \mathrm{Ci}(8231$ neutrons/s) \\
\hline Calculated efficiency & $17(1) \%$ \\
\hline
\end{tabular}

A second efficiency measurement was made using a larger neutron source placed in the center of the detector system. Details of the run are given in Table 3.2. Data was accumulated both with the VME system and with an AMSR 150 shift register that had no dead time. The two results agreed with each other, 17\% (VME system) and 16.7\% (shift register).

Table 3.2. Source run parameters from VME system (December 10, 2012)

\begin{tabular}{|l|l|}
\hline \multicolumn{1}{|c|}{ Parameter } & \multicolumn{1}{c|}{ Value } \\
\hline Events recorded & 50000 \\
\hline Run time & $76.9 \mathrm{~s}$ \\
\hline Dead time & $69.7 \mathrm{~s}(90.6 \%)$ \\
\hline Average time between pulses & $146 \mathrm{us}(6849 \mathrm{cps})$ \\
\hline Source Activity (\#60208-44) & $\sim 9.5 \mu \mathrm{Ci}(40980$ neutrons $/ \mathrm{s})$ \\
\hline Shift Register cps & $6825 \mathrm{cps}$ \\
\hline Calculated efficiency & $17(1) \%$ \\
\hline
\end{tabular}

Thus the efficiency of the detector is about $17 \%$. This compares reasonably with the previous MCNP model prediction of $19.9 \%$.

Spallation events can produce very large numbers of neutrons. The data presented in this paper shows multiplicities of above 20 for some materials. Given the efficiency for the detector, the events where 20 neutrons are detected must represent very large numbers of neutrons emitted in the spallation event and subsequent cascades. 


\section{Muon Measurements}

The rate of cosmic rays hitting the Earth surface is dependent on a variety of factors. These factors can be separated into the ones that affect the development of the cosmic ray shower and those factors that affect the number of primaries impinging the atmosphere. In the first category, factors such as barometric pressure and geomagnetic rigidity at the point of observation are very important. The measurements presented in this work were made at $\sim 30 \mathrm{~m}$ above sea-level, so they should be representative of sea-level exposures. In the second category, the astronomical solar cycle is the most prominent one affecting the cosmic ray shower rate. Variations in solar activity can vary the cosmic ray rate by as much as $20 \%$, as shown in Figure $4-1$. In order to correlate the experimental runs with the cosmic ray rate over the time interval when the neutron counting was performed, a second detector was used to log the muon rate in the vicinity of the multiplicity counter. Muons are part of the cosmic ray shower, so logging the rate of muons indicated that there were no changes is the overall cosmic ray rate due to a change in solar activity. The $\mu$ Witness detector, described in [Aguayo 2012a], and shown in Figure 4-2, was used during the experimental runs. During the data taking activity presented in this work the muon rate varied $6 \%$ at most, as listed in Table 1.2 .

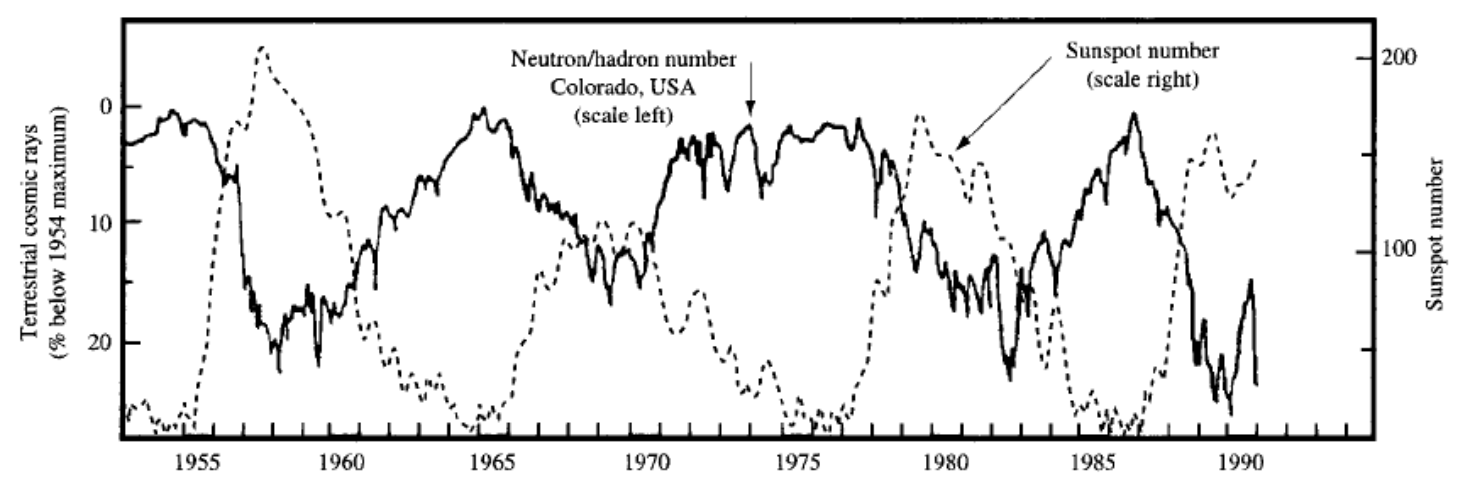

Figure 4-1. Cosmic ray fluctuation in a 35 year time period, from [Ziegler 1998]

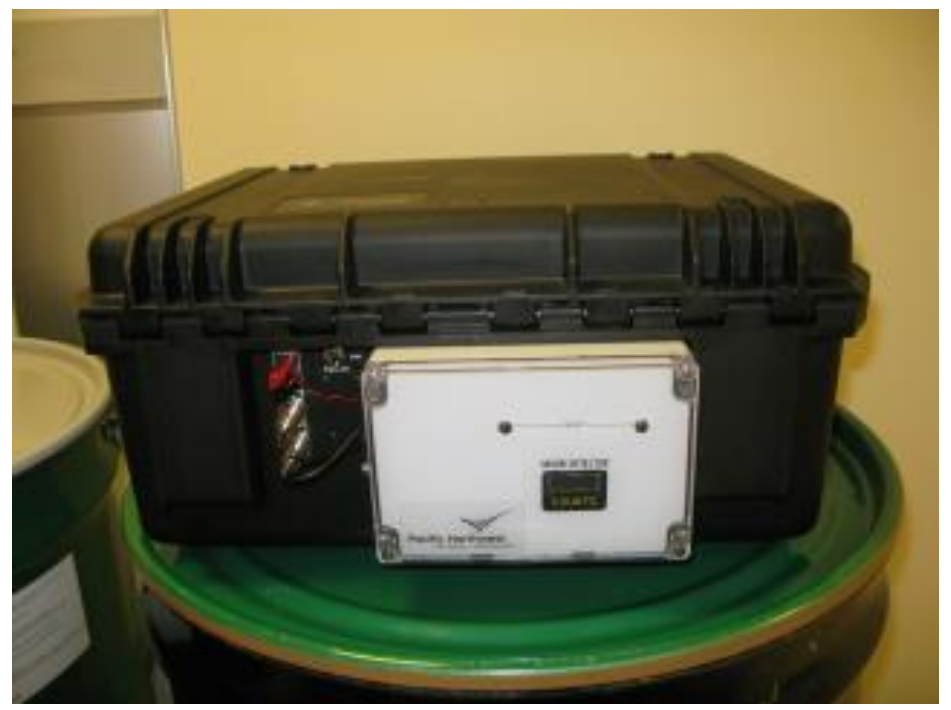

Figure 4-2. The uWitness detector [Aguayo 2012a]. 


\section{Background Measurements}

The background neutrons (with the wood support in place) were measured before and after most measurements of material to assure that no significant changes occurred. Figure 5-1 shows the distribution of neutron multiplicity in cosmic background events measured by the detector system without any target material. Panel a) shows multiplicity in a $100 \mathrm{~ms}$ time window, panel b) shows multiplicity in a $10 \mathrm{~ms}$ time window, panel c) shows multiplicity in a $1 \mathrm{~ms}$ time window and panel d) shows multiplicity in a $100 \mu$ s time window. The measured data are the black lines, while the red line is a Poisson distribution with the same mean value as the data (both a histogram and a smooth fit are shown). It is seen that for all time windows, the multiplicity data follow a Poisson distribution as would be expected for background.

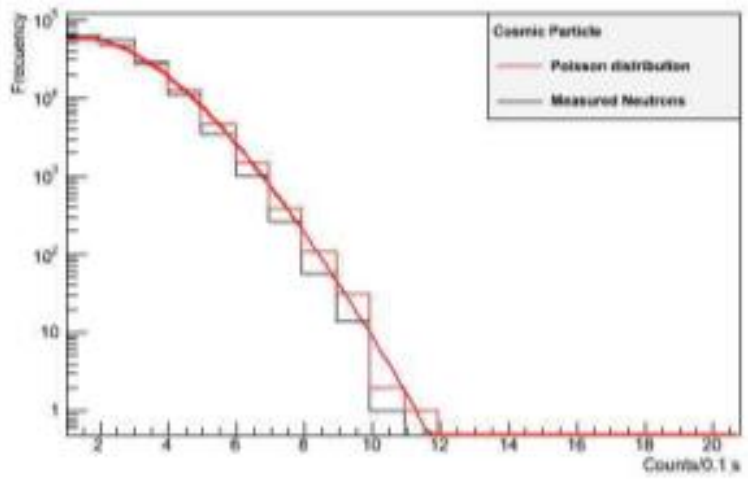

a) $100 \mathrm{~ms}$

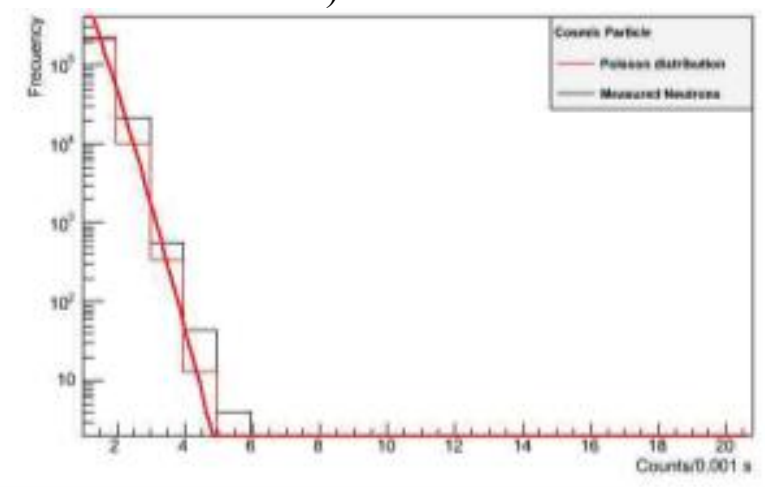

c) $1 \mathrm{~ms}$

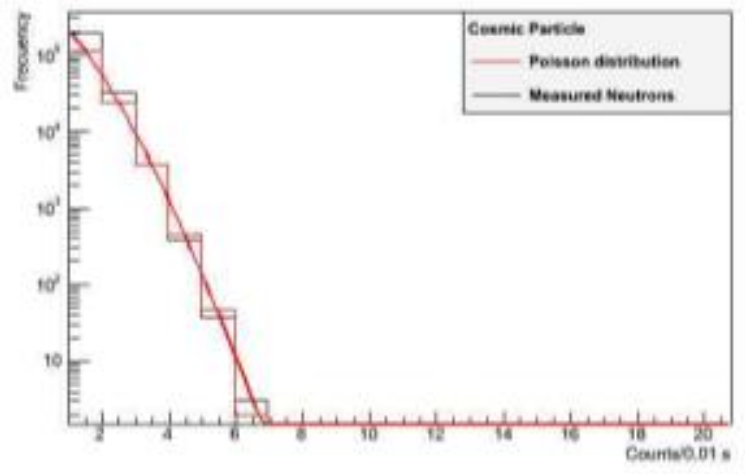

b) $10 \mathrm{~ms}$

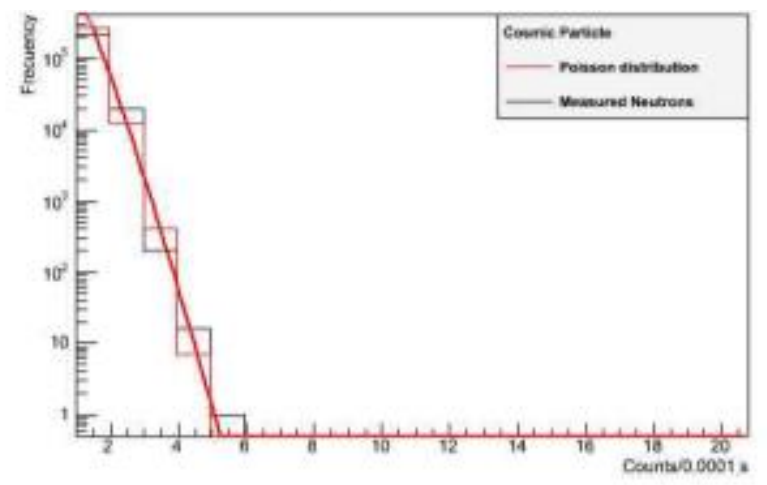

d) $0.1 \mathrm{~ms}$

Figure 5-1. Distribution of neutron multiplicity events caused by cosmic neutrons without a target.

Table 5.1 presents information on the analysis of six of the background runs. The table presents the gross counts, the number of counts above what a Poisson distribution would predict, and the number of counts with a specified value of multiplicity and above. The data for a time window of $1 \mathrm{~ms}$ is most relevant for analysis. For the $1 \mathrm{~ms}$ time window, only a small percentage of counts show up with multiplicities of four or more. For the $1 \mathrm{~ms}$ window and multiplicity of four or more, the average value was 58.5 counts, or $9(1) \times 10^{-4} \mathrm{cps}$. This can be compared to the results obtained for the various target materials. 
Table 5.1. Background run results (12/10/2012)

\begin{tabular}{|c|c|c|c|c|c|c|c|}
\hline $\begin{array}{l}\text { Gate } \\
\text { length }(\mathrm{ms})\end{array}$ & $\begin{array}{l}\text { Total } \\
\text { counts }\end{array}$ & $\begin{array}{l}\text { Counts }> \\
\text { Poisson } \\
\end{array}$ & $\begin{array}{l}\text { Integral } \\
\text { Mult. }>4\end{array}$ & $\begin{array}{l}\text { Integral } \\
\text { Mult. }>5\end{array}$ & $\begin{array}{l}\text { Integral } \\
\text { Mult. }>6 \\
\end{array}$ & $\begin{array}{l}\text { Integral } \\
\text { Mult. }>7\end{array}$ & $\begin{array}{l}\text { Integral } \\
\text { Mult. }>8\end{array}$ \\
\hline \multicolumn{8}{|c|}{ Data run of $12 / 10 / 12$} \\
\hline 100 & 195819 & 105 & 19193 & 5897 & 1610 & 411 & 87 \\
\hline 10 & 271725 & 8 & 503 & 51 & 5 & 0 & 0 \\
\hline 1 & 282756 & 50 & 55 & 5 & 0 & 0 & 0 \\
\hline 0.1 & 284046 & 11 & 18 & 1 & 0 & 0 & 0 \\
\hline \multicolumn{8}{|c|}{ Data run of $1 / 6 / 13$} \\
\hline 100 & 191250 & 2511 & 21599 & 6975 & 2013 & 499 & 100 \\
\hline 10 & 270656 & 90 & 601 & 57 & 8 & 3 & 2 \\
\hline 1 & 282542 & 63 & 68 & 10 & 6 & 3 & 2 \\
\hline 0.1 & 283911 & 11 & 21 & 3 & 2 & 0 & 0 \\
\hline \multicolumn{8}{|c|}{ Data run of $1 / 10 / 13$} \\
\hline 100 & 195088 & 852 & 19940 & 6042 & 1674 & 420 & 87 \\
\hline 10 & 271243 & 21 & 532 & 50 & 7 & 1 & 1 \\
\hline 1 & 282255 & 55 & 63 & 9 & 2 & 1 & 1 \\
\hline 0.1 & 283659 & 11 & 21 & 3 & 0 & 0 & 0 \\
\hline \multicolumn{8}{|c|}{ Data run of $1 / 13 / 13$} \\
\hline 100 & 198035 & 864 & 18318 & 5485 & 1363 & 348 & 80 \\
\hline 10 & 271685 & 103 & 544 & 61 & 4 & 1 & 1 \\
\hline 1 & 282181 & 49 & 61 & 8 & 2 & 1 & 1 \\
\hline 0.1 & 283501 & 13 & 23 & 5 & 1 & 0 & 0 \\
\hline \multicolumn{8}{|c|}{ Data run of $1 / 15 / 13$} \\
\hline 100 & 198365 & 1032 & 18056 & 5358 & 1406 & 351 & 73 \\
\hline 10 & 271853 & 54 & 457 & 40 & 6 & 2 & 2 \\
\hline 1 & 282414 & 39 & 46 & 6 & 3 & 2 & 1 \\
\hline 0.1 & 283686 & 3 & 10 & 2 & 1 & 0 & 0 \\
\hline \multicolumn{8}{|c|}{ Data run of $1 / 18 / 13$} \\
\hline 100 & 195956 & 311 & 19399 & 5780 & 1537 & 356 & 72 \\
\hline 10 & 271347 & 51 & 460 & 47 & 3 & 0 & 0 \\
\hline 1 & 282239 & 51 & 58 & 5 & 0 & 0 & 0 \\
\hline 0.1 & 283508 & 3 & 12 & 2 & 0 & 0 & 0 \\
\hline
\end{tabular}

The data in Figure 5-2 show the neutron and muon background rates that were measured during the time period that most of the various target materials were being counted. Data values are listed in Table 1.2. The background neutron rate remained fairly constant at 9.6(4) counts per second and the muon rate was constant at 2.28(5) counts per second. 


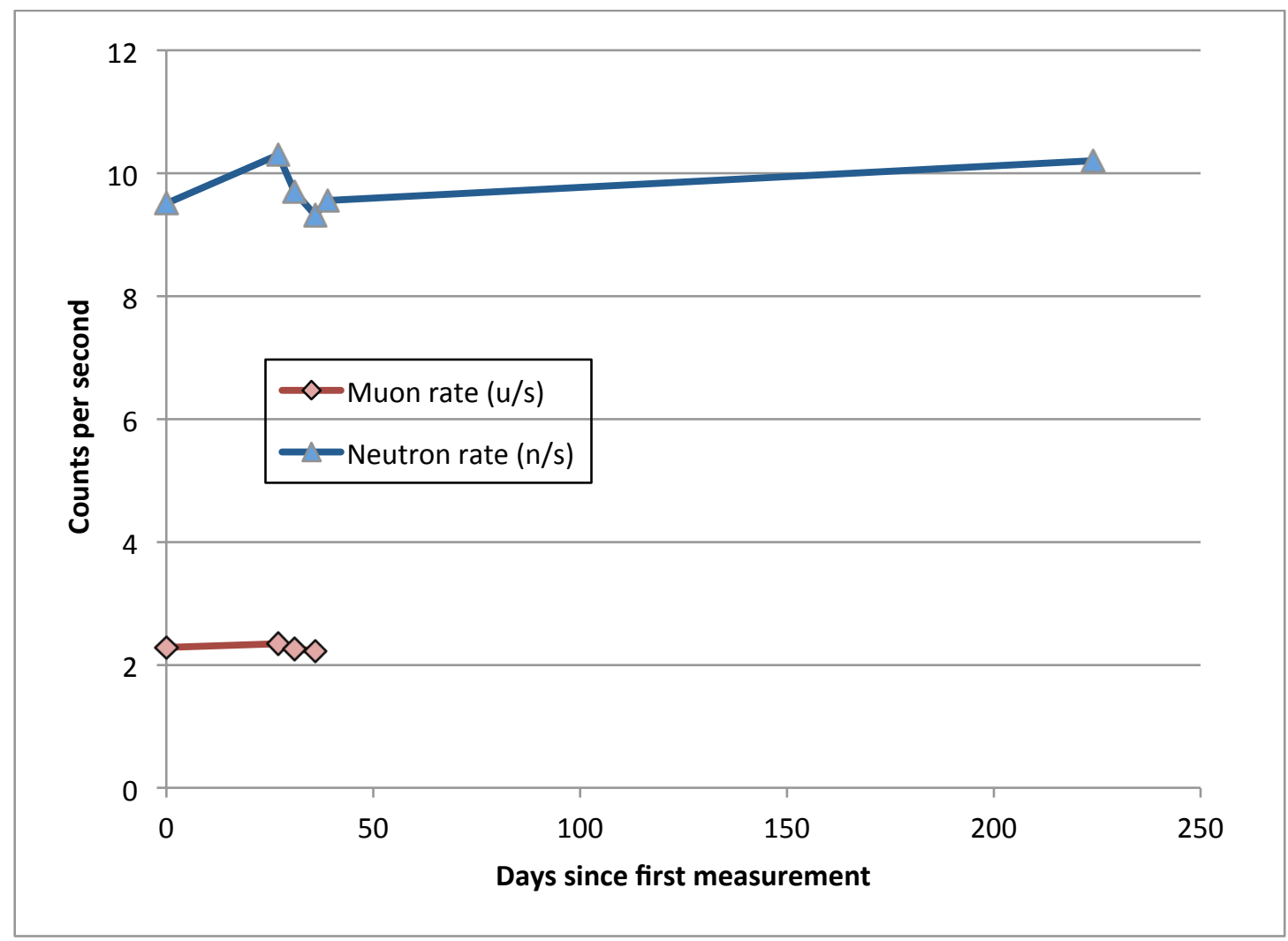

Figure 5-2. Background rates recorded before and after the runs using the different materials as a target. 


\section{Polyethylene Run}

Polyethylene was the lowest atomic number material measured, and it produces the smallest ship effect signature in terms of multiplicity. Polyethylene commonly has the chemical formula $\left(\mathrm{C}_{2} \mathrm{H}_{4}\right)_{\mathrm{n}} \mathrm{H}_{2}$. The high-density polyethylene (HDPE) used in these measurements has a density of about $0.94 \mathrm{~g} / \mathrm{cm}^{3}$. The material had dimensions of $30.5 \mathrm{~cm}$ by $30.5 \mathrm{~cm}$ by $30.5 \mathrm{~cm}$ and a total mass as listed in Table 1.1. The polyethylene sample consisted of 12 identical $2.54 \mathrm{~cm}$ thick slabs. Figure 6-1 shows the polyethylene in place on top of the wooden support inside of the multiplicity counter with one side moved (the support wood was included in the background).

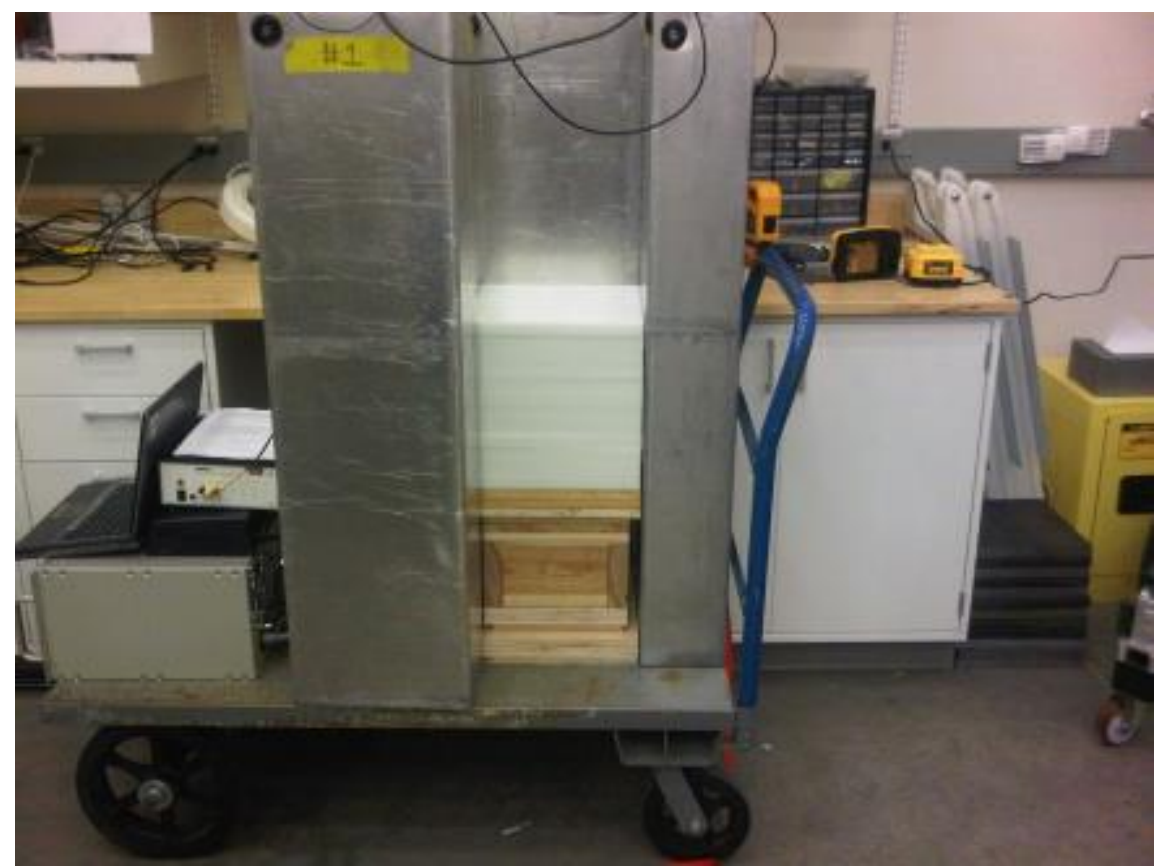

Figure 6-1. Multiplicity counter open showing the HDPE in position for measurement.

The data in Figure 6-2 show the distribution of neutron multiplicity in cosmic induced events measured by the detector system with a polyethylene target mass. Panel a) shows multiplicity in a $100 \mathrm{~ms}$ time window, panel b) shows multiplicity in a $10 \mathrm{~ms}$ time window, panel c) shows multiplicity in a $1 \mathrm{~ms}$ time window and panel d) shows multiplicity in a $100 \mu$ s time window. This target material will have an impact on the neutron die away time of the detector, therefore the gate length should accommodate a longer die away times. In the measurements presented in this section, even in the case of the shortest time window, $0.1 \mathrm{~ms}$, there is no apparent leakage of events in the larger multiplicity windows. This could be an indication of an artifact due to larger die away times in the detector, especially when loaded with the polyethylene target material. The measured data are the black lines, while the red line is a Poisson distribution with the same mean value as the data. It is seen that for long time windows, the multiplicity data follow a Poisson distribution as would be expected. For shorter time windows, there are a few excess events at higher multiplicity, indicating a few spallation events from the polyethylene. 


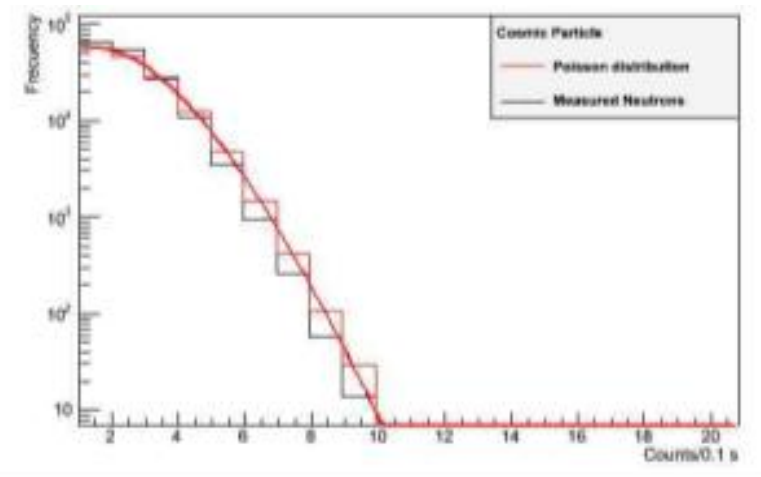

a) $100 \mathrm{~ms}$

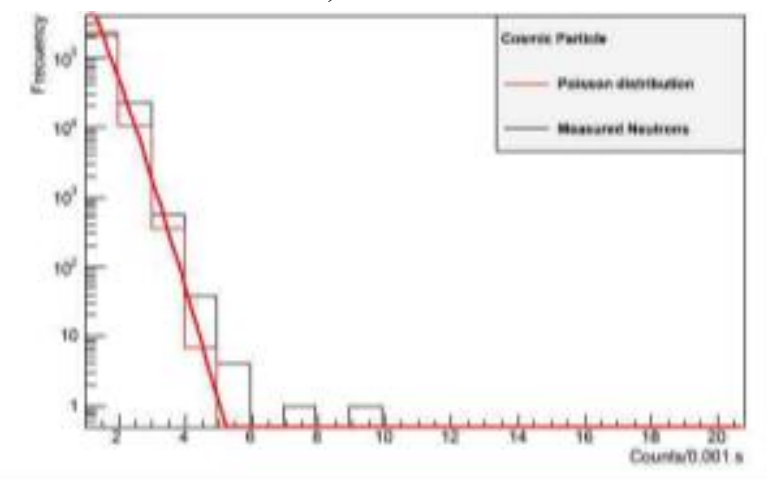

c) $1 \mathrm{~ms}$

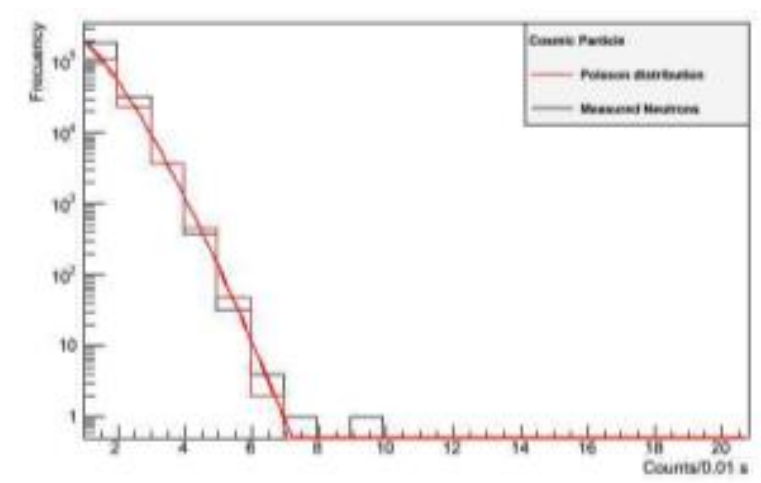

b) $10 \mathrm{~ms}$

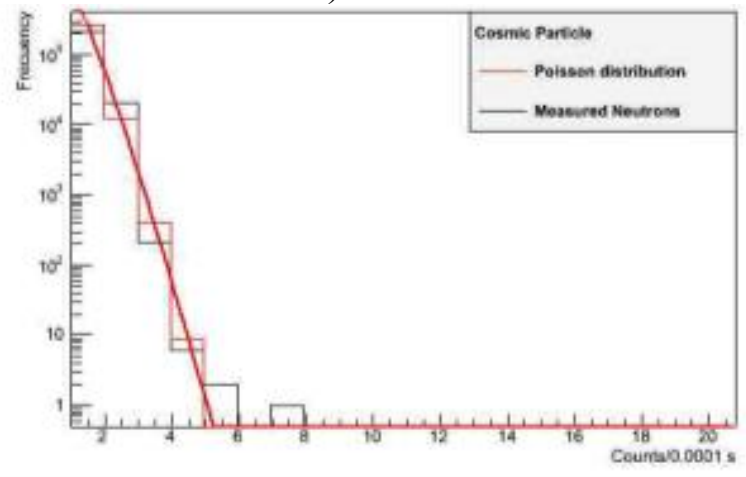

d) $0.1 \mathrm{~ms}$

Figure 6-2. Distribution of neutron multiplicity caused by cosmic neutrons in polyethylene.

The data in Table 6.1 show the results for the measurement of the HDPE. The count rates for HDPE are small, and only slightly different from background rates. Considering the $1 \mathrm{~ms}$ time window, the 68 events with a multiplicity of four or more obtained in a run time of $71321 \mathrm{~s}$ gives a net rate of $3(10) \times 10^{-5} \mathrm{cps}$, using the average background rate and estimating the uncertainty as the same as that seen in the background average. For the second run, the 49 events with a multiplicity of four or more obtained in a run time of 70003 s gives a net rate of $2(1) \times 10^{-4} \mathrm{cps}$, using the average background rate and estimating the uncertainty as the standard deviation between measurements.

Table 6.1. Polyethylene run results $(1 / 14 / 2013)$

\begin{tabular}{|l|l|l|l|l|l|l|l|}
\hline $\begin{array}{l}\text { Gate length } \\
(\mathbf{m s})\end{array}$ & $\begin{array}{l}\text { Total } \\
\text { counts }\end{array}$ & $\begin{array}{l}\text { Counts }> \\
\text { Poisson }\end{array}$ & $\begin{array}{l}\text { Integral } \\
\text { Mult. }>\text { 4 }\end{array}$ & $\begin{array}{l}\text { Integral } \\
\text { Mult. }>5\end{array}$ & $\begin{array}{l}\text { Integral } \\
\text { Mult. }>\mathbf{6}\end{array}$ & $\begin{array}{l}\text { Integral } \\
\text { Mult. }>\text { 7 }\end{array}$ & $\begin{array}{l}\text { Integral } \\
\text { Mult. }>8\end{array}$ \\
\hline Data run of 1/4/13 & 272626 & 82 & 429 & 45 & 5 & 3 & 1 \\
\hline 100 & 202542 & 2741 & 16347 & 4612 & 1136 & 268 & 55 \\
\hline 10 & 282334 & 55 & 68 & 13 & 6 & 2 & 1 \\
\hline 1 & 283593 & 9 & 24 & 7 & 3 & 1 & 1 \\
\hline 0.1 & \multicolumn{7}{|l|}{} \\
\hline Data run of 1/20/13 & 272264 & 42 & 433 & 40 & 2 & 1 & 0 \\
\hline 100 & 201643 & 2286 & 16802 & 4856 & 1194 & 243 & 55 \\
\hline 10 & 282267 & 42 & 49 & 11 & 0 & 0 & 0 \\
\hline 1 & 283441 & 2 & 15 & 3 & 1 & 0 & 0 \\
\hline 0.1 & & & & & & \\
\hline
\end{tabular}




\section{Aluminum Run}

Aluminum was measured in the same manner as the polyethylene. The target material had dimensions of $30.5 \mathrm{~cm}$ by $30.5 \mathrm{~cm}$ by $30.5 \mathrm{~cm}$ and a total mass as listed in Table 1.1 . The aluminum was in the form of 16 bricks, each brick with dimensions of $30.5 \times 7.5 \times 7.5 \mathrm{~cm}^{3}$. Figure 7-1 shows the aluminum in place on top of the wooden support inside of the multiplicity counter with one side moved.

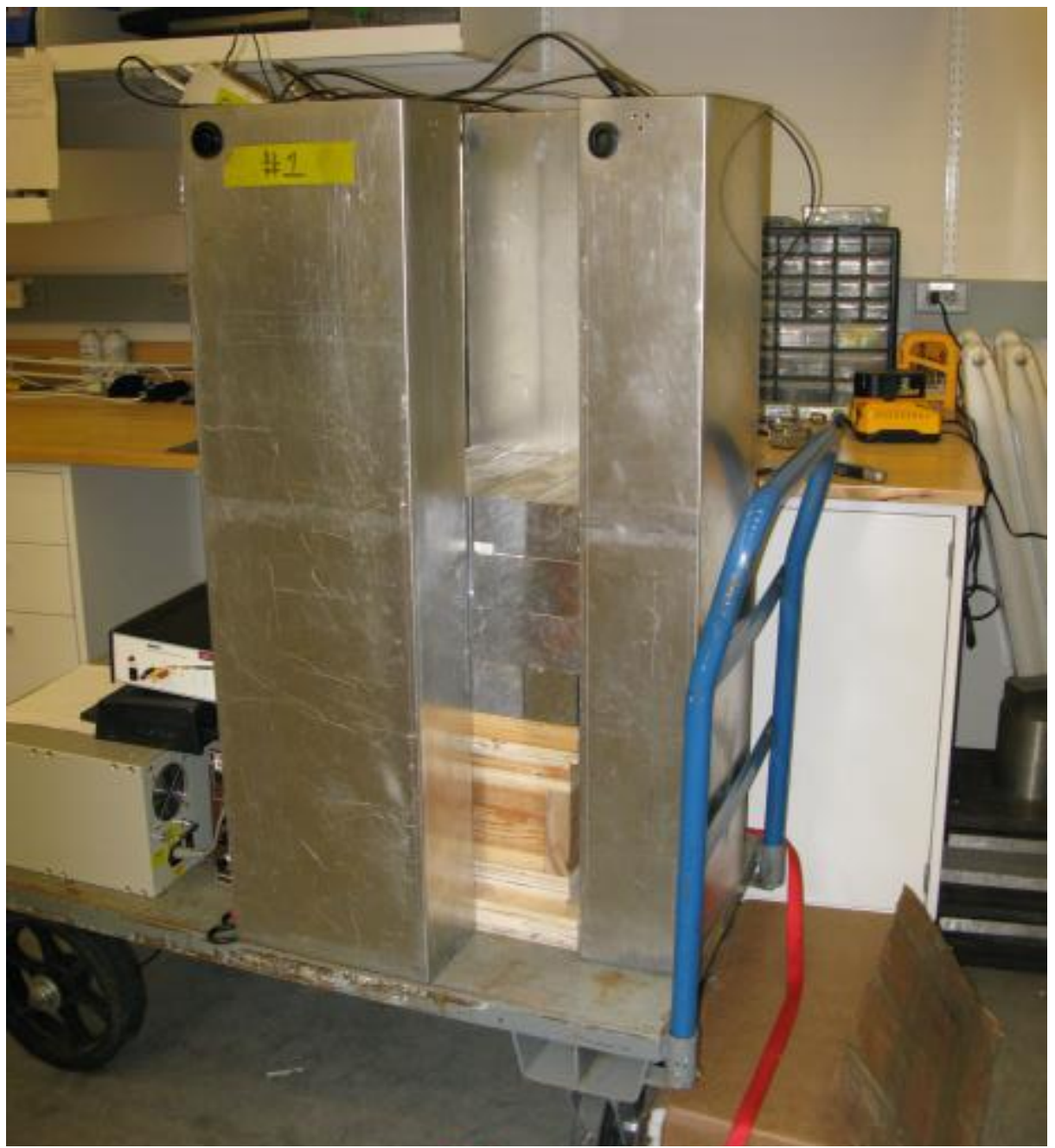

Figure 7-1. Multiplicity counter open showing the aluminum in position for measurement

The data in Figure 7-2 show the distribution of neutron multiplicity in cosmic induced events measured by the detector system with an aluminum target mass. Panel a) shows multiplicity in a $100 \mathrm{~ms}$ time window, panel b) shows multiplicity in a $10 \mathrm{~ms}$ time window, panel c) shows multiplicity in a $1 \mathrm{~ms}$ time window and panel d) shows multiplicity in a $100 \mu$ s time window. The measured data are the black lines, while the red line is a Poisson distribution with the same mean value as the data. It is seen that for most time windows, the multiplicity data deviate somewhat from a Poisson distribution. 


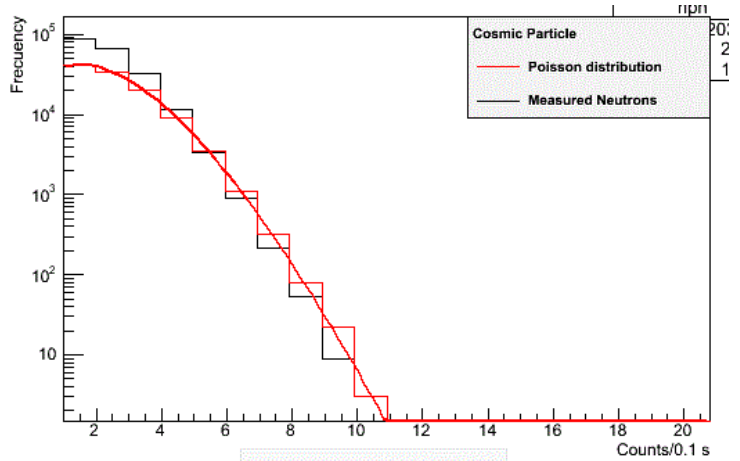

a) $100 \mathrm{~ms}$

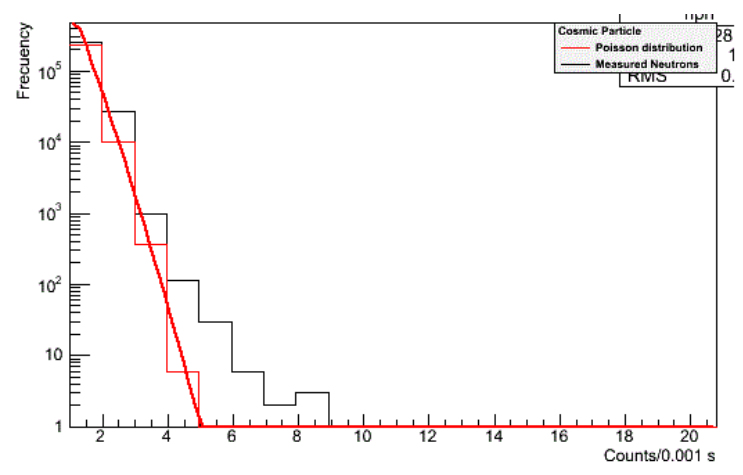

c) $1 \mathrm{~ms}$

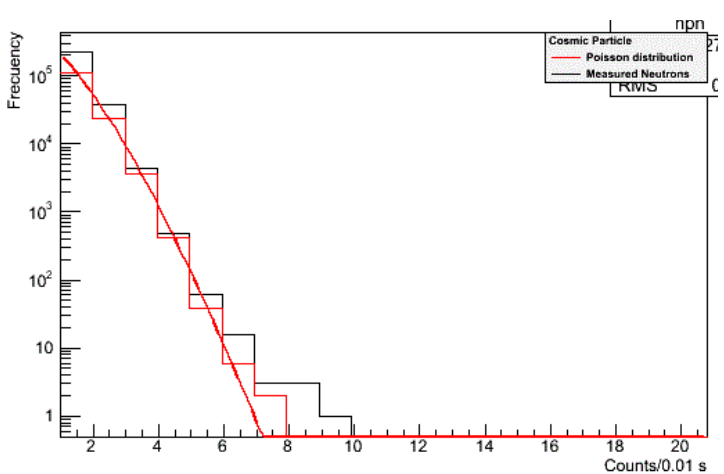

b) $10 \mathrm{~ms}$

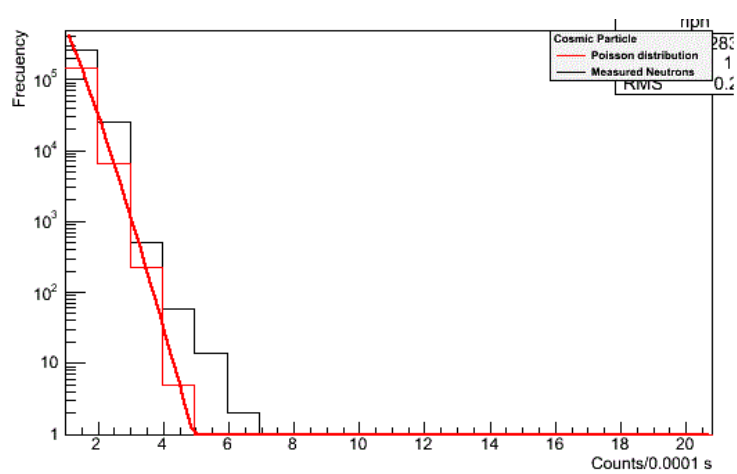

d) $0.1 \mathrm{~ms}$

Figure 7-2. Distribution of neutron multiplicity caused by cosmic neutrons in aluminum.

The data in Table 7.1 show the results for the measurement of the aluminum sample.

Considering the $1 \mathrm{~ms}$ time window, the 157 events with a multiplicity of four or more obtained in a total run time of $70,985 \mathrm{~s}$ gives a net rate of $1.3(4) \times 10^{-3} \mathrm{cps}$, using the average background rate and estimating the uncertainty as the same as that seen in the background average.

Table 7.1. Aluminum run results $(3 / 1 / 2013)$

\begin{tabular}{|l|l|l|l|l|l|l|l|}
\hline $\begin{array}{l}\text { Gate length } \\
(\mathbf{m s})\end{array}$ & $\begin{array}{l}\text { Total } \\
\text { counts }\end{array}$ & $\begin{array}{l}\text { Counts }> \\
\text { Poisson }\end{array}$ & $\begin{array}{l}\text { Integral } \\
\text { Mult. }>\text { 4 }\end{array}$ & $\begin{array}{l}\text { Integral } \\
\text { Mult. }>\mathbf{5}\end{array}$ & $\begin{array}{l}\text { Integral } \\
\text { Mult. }>\mathbf{6}\end{array}$ & $\begin{array}{l}\text { Integral } \\
\text { Mult. }>\text { 7 }\end{array}$ & $\begin{array}{l}\text { Integral } \\
\text { Mult. }>\text { 8 }\end{array}$ \\
\hline 100 & 203054 & 2297 & 16305 & 4574 & 1179 & 293 & 65 \\
\hline 10 & 271935 & 53 & 565 & 84 & 23 & 7 & 4 \\
\hline 1 & 281560 & 150 & 157 & 41 & 11 & 5 & 3 \\
\hline 0.1 & 283032 & 67 & 74 & 16 & 2 & 0 & 0 \\
\hline
\end{tabular}




\section{Steel Run}

Steel was measured in the same manner as the polyethylene. The material had dimensions of $30.5 \mathrm{~cm}$ by $30.5 \mathrm{~cm}$ by $30.5 \mathrm{~cm}$ and a total mass as listed in Table 1.1 . The steel consisted of 12 identical $2.54 \mathrm{~cm}$ thick slabs. Figure 8-1 shows the steel in place on top of the wooden support inside of the multiplicity counter with one side moved.

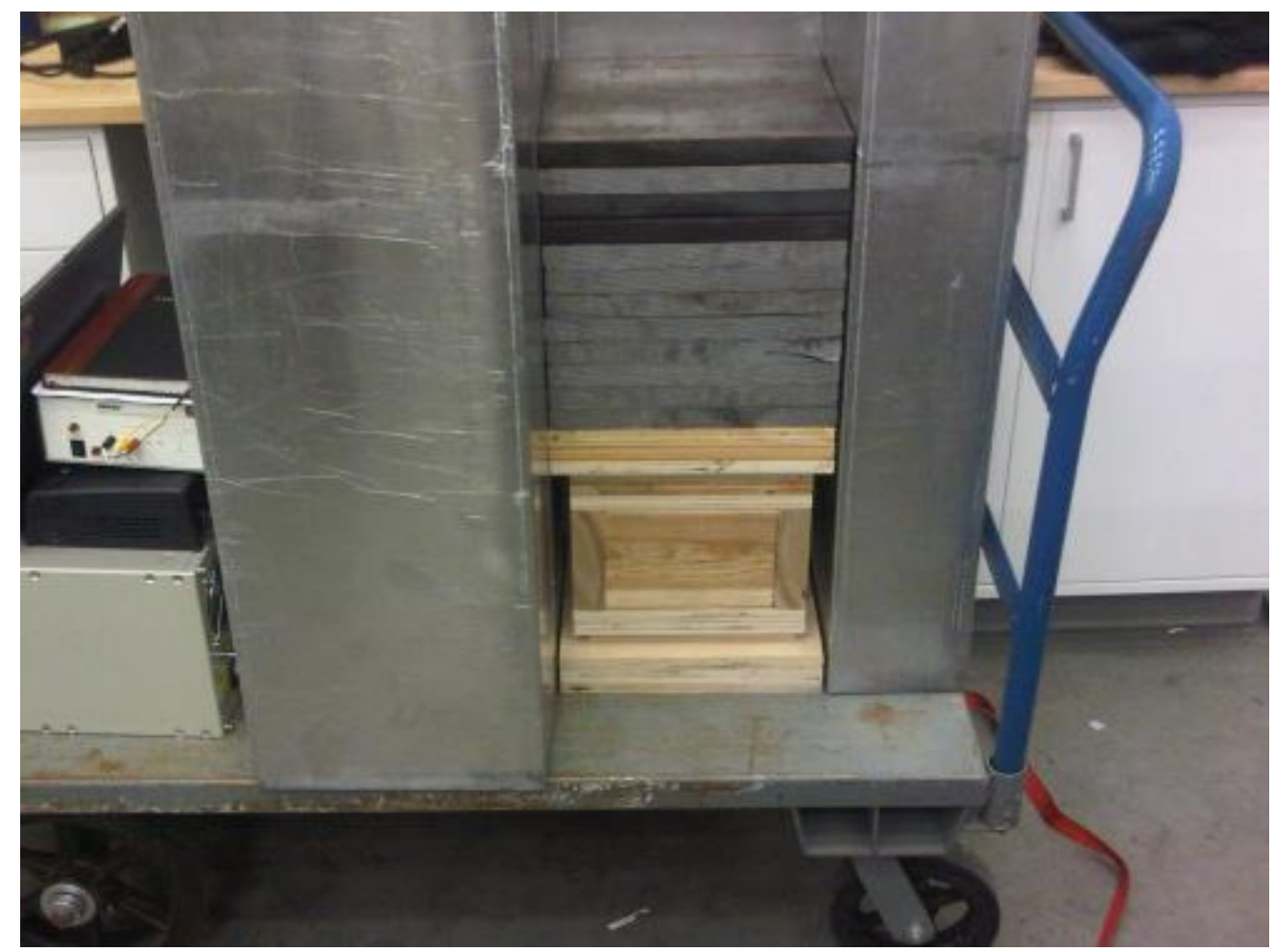

Figure 8-1. Multiplicity counter open showing the iron in position for measurement.

The data in Figure 8-2 show the distribution of neutron multiplicity in cosmic induced events measured by the detector system with a steel target mass. Panel a) shows multiplicity in a $100 \mathrm{~ms}$ time window, panel b) shows multiplicity in a $10 \mathrm{~ms}$ time window, panel c) shows multiplicity in a $1 \mathrm{~ms}$ time window and panel d) shows multiplicity in a $100 \mu \mathrm{s}$ time window. The measured data are the black lines, while the red line is a Poisson distribution with the same mean value as the data. It is seen that for all time windows, the multiplicity data deviate from a Poisson distribution since there are a sufficient number of spallation events in steel to be easily observed. 


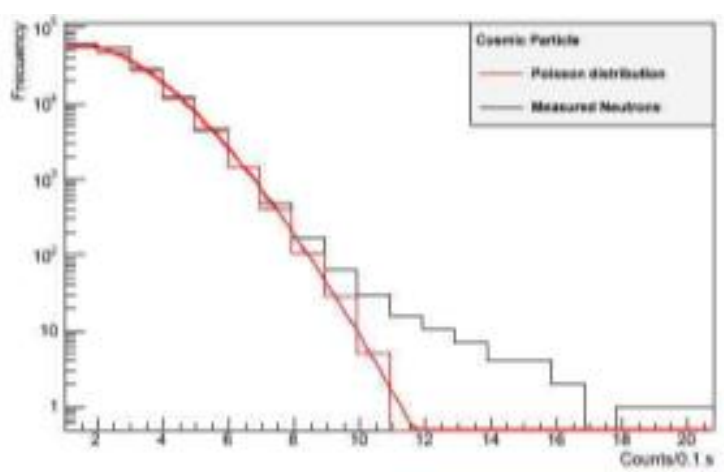

a) $100 \mathrm{~ms}$

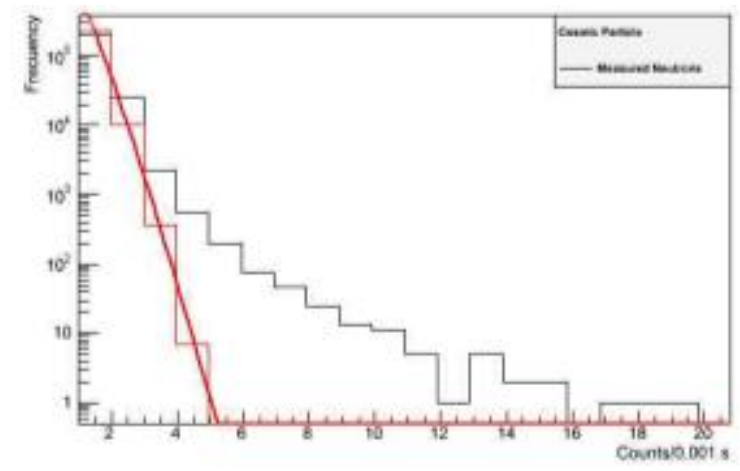

c) $1 \mathrm{~ms}$

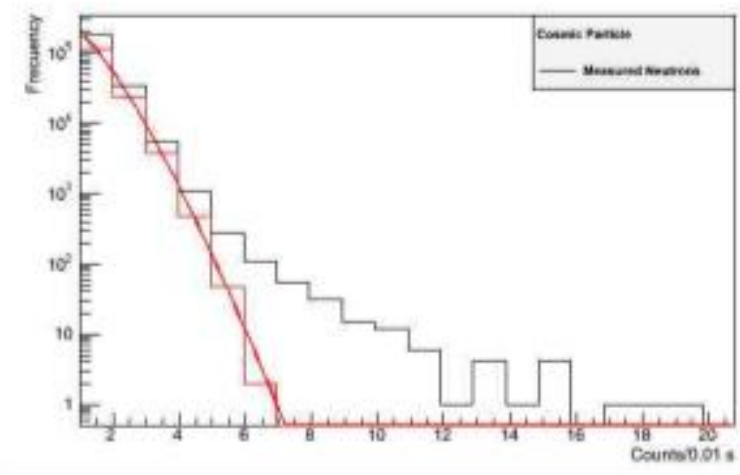

b) $10 \mathrm{~ms}$

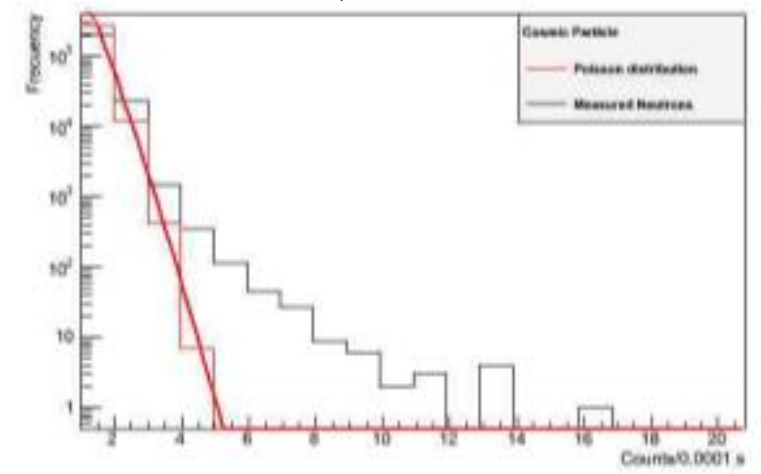

d) $0.1 \mathrm{~ms}$

Figure 8-2. Distribution of neutron multiplicity caused by cosmic neutrons in steel.

The data in Table 8.1 show the results for the measurement of the steel cube. Considering the 1 ms time window, the 1212 events with a multiplicity of four or more obtained in a total run time of $60,780 \mathrm{~s}$ gives a net rate of $1.9(4) \times 10^{-2} \mathrm{cps}$, using the average background rate and estimating the uncertainty as the same as that seen in the background average.

Table 8.1. Steel run results $(1 / 11 / 2013)$

\begin{tabular}{|l|l|l|l|l|l|l|l|}
\hline $\begin{array}{l}\text { Gate length } \\
(\mathbf{m s})\end{array}$ & $\begin{array}{l}\text { Total } \\
\text { counts }\end{array}$ & $\begin{array}{l}\text { Counts }> \\
\text { Poisson }\end{array}$ & $\begin{array}{l}\text { Integral } \\
\text { Mult. }>\text { 4 }\end{array}$ & $\begin{array}{l}\text { Integral } \\
\text { Mult. }>\mathbf{5}\end{array}$ & $\begin{array}{l}\text { Integral } \\
\text { Mult. }>\mathbf{6}\end{array}$ & $\begin{array}{l}\text { Integral } \\
\text { Mult. }>\text { 7 }\end{array}$ & $\begin{array}{l}\text { Integral } \\
\text { Mult. }>\text { 8 }\end{array}$ \\
\hline 100 & 190552 & 399 & 22723 & 8198 & 2784 & 1018 & 399 \\
\hline 10 & 265377 & 1477 & 1988 & 679 & 302 & 168 & 95 \\
\hline 1 & 276174 & 1206 & 1212 & 490 & 260 & 151 & 86 \\
\hline 0.1 & 279076 & 717 & 726 & 286 & 136 & 69 & 32 \\
\hline
\end{tabular}




\section{Copper Run}

Copper was measured in the same manner as the polyethylene. The material had dimensions of $30.5 \mathrm{~cm}$ by $30.5 \mathrm{~cm}$ by $30.5 \mathrm{~cm}$ and a total mass as listed in Table 1.1. The copper consisted of six identical $5.1 \mathrm{~cm}$ thick slabs. Figure 9-1 shows the copper in place on top of the wooden support inside of the multiplicity counter with one side moved.

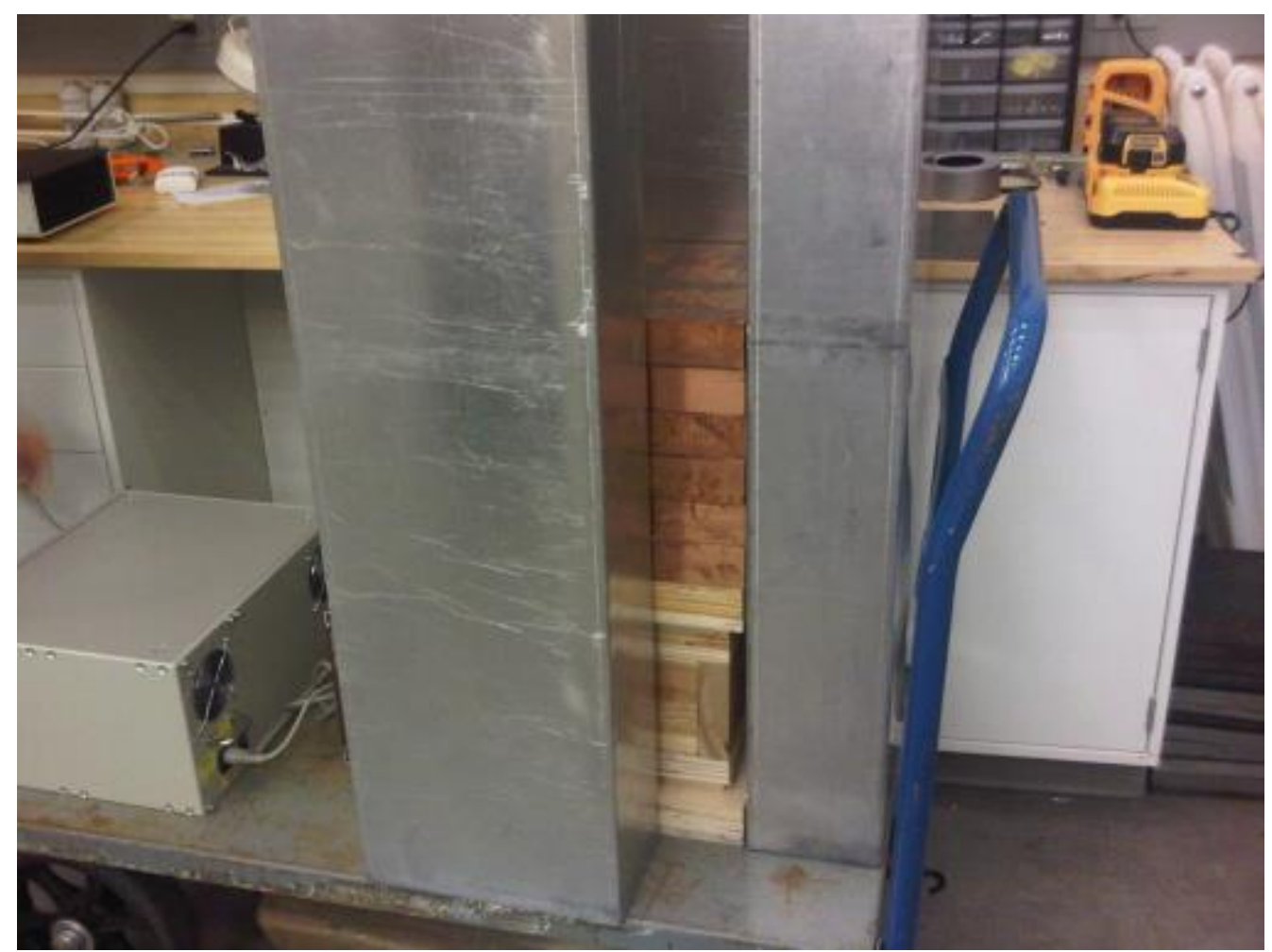

Figure 9-1. Multiplicity counter open showing the copper in position for measurement.

The data in Figure 9-2 show the distribution of neutron multiplicity in cosmic induced events measured by the detector system with a copper target mass. Panel a) shows multiplicity in a 100 ms time window, panel b) shows multiplicity in a $10 \mathrm{~ms}$ time window, panel c) shows multiplicity in a $1 \mathrm{~ms}$ time window and panel d) shows multiplicity in a $100 \mu$ s time window. The measured data are the black lines, while the red line is a Poisson distribution with the same mean value as the data. It is seen that for all time windows, the multiplicity data deviate from a Poisson distribution since there are a sufficient number of spallation events in copper to be easily observed. 


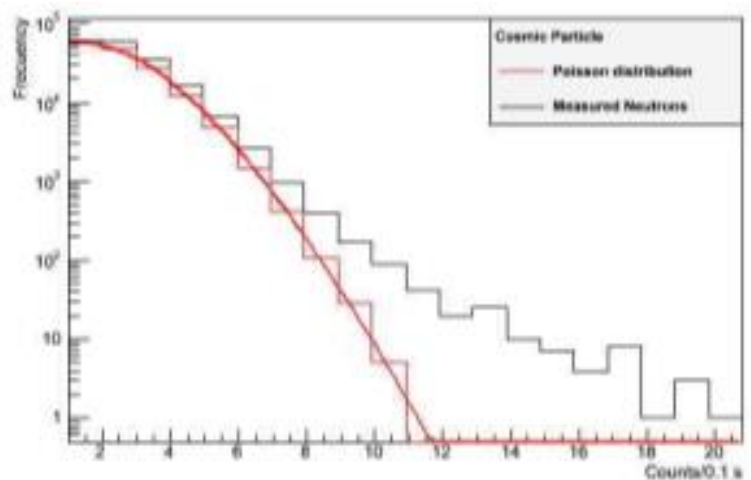

a) $100 \mathrm{~ms}$

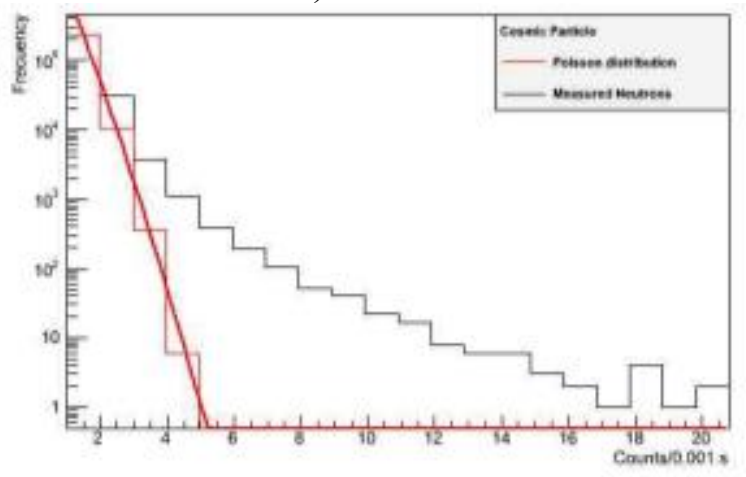

c) $1 \mathrm{~ms}$

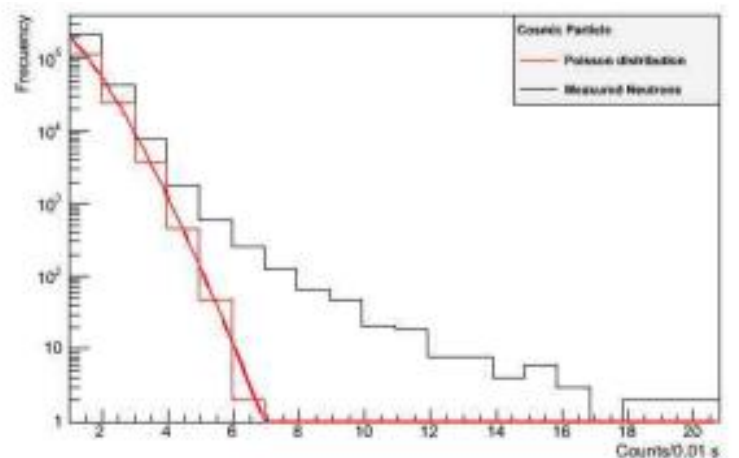

b) $10 \mathrm{~ms}$

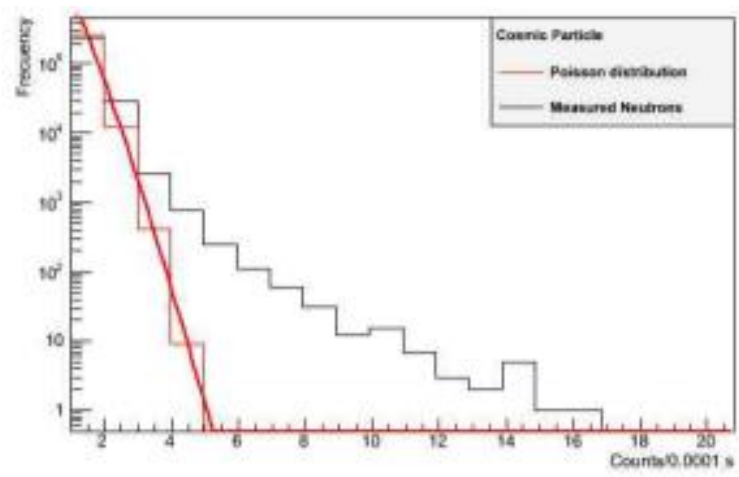

d) $0.1 \mathrm{~ms}$

Figure 9-2. Distribution of neutron multiplicity caused by cosmic neutrons in copper.

The data in Table 9.1 show the results for the measurement of the copper. Considering the $1 \mathrm{~ms}$ time window, the average of 1981 events with a multiplicity of four or more obtained in an average run time of 54,076 sives a net rate of $3.6(3) \times 10^{-2} \mathrm{cps}$, using the average background rate and estimating the uncertainty as the standard deviation between measurements. 
Table 9.1. Copper run results

\begin{tabular}{|c|c|c|c|c|c|c|c|}
\hline $\begin{array}{l}\text { Gate length } \\
\text { (ms) }\end{array}$ & $\begin{array}{l}\text { Total } \\
\text { counts }\end{array}$ & $\begin{array}{l}\text { Counts }> \\
\text { Poisson }\end{array}$ & $\begin{array}{l}\text { Integral } \\
\text { Mult. }>4\end{array}$ & $\begin{array}{l}\text { Integral } \\
\text { Mult. }>5\end{array}$ & $\begin{array}{l}\text { Integral } \\
\text { Mult. }>6\end{array}$ & $\begin{array}{l}\text { Integral } \\
\text { Mult. }>7\end{array}$ & $\begin{array}{l}\text { Integral } \\
\text { Mult. }>8\end{array}$ \\
\hline \multicolumn{8}{|c|}{ Data run of $12 / 12 / 12$} \\
\hline 100 & 181886 & 8148 & 27236 & 11024 & 4380 & 1757 & 786 \\
\hline 10 & 261277 & 2476 & 2987 & 1177 & 576 & 317 & 188 \\
\hline 1 & 273275 & 1953 & 1958 & 854 & 463 & 267 & 165 \\
\hline 0.1 & 276865 & 1245 & 1255 & 493 & 247 & 136 & 77 \\
\hline \multicolumn{8}{|c|}{ Data run of $12 / 13 / 12$} \\
\hline 100 & 182336 & 8021 & 27109 & 11113 & 4349 & 1746 & 781 \\
\hline 10 & 261011 & 2678 & 3189 & 1231 & 552 & 322 & 176 \\
\hline 1 & 272937 & 2093 & 2099 & 921 & 447 & 267 & 151 \\
\hline 0.1 & 276622 & 1315 & 1324 & 533 & 252 & 127 & 60 \\
\hline \multicolumn{8}{|c|}{ Data run of $12 / 14 / 12$} \\
\hline 100 & 180627 & 8819 & 27907 & 11301 & 4411 & 1728 & 762 \\
\hline 10 & 261163 & 2454 & 2965 & 1123 & 522 & 264 & 156 \\
\hline 1 & 273370 & 1907 & 1913 & 834 & 409 & 226 & 135 \\
\hline 0.1 & 277003 & 1212 & 1221 & 475 & 227 & 108 & 71 \\
\hline \multicolumn{8}{|c|}{ Data run of $12 / 18 / 12$} \\
\hline 100 & 180838 & 8645 & 27733 & 11410 & 4654 & 1890 & 862 \\
\hline 10 & 260379 & 2747 & 3258 & 1264 & 604 & 344 & 222 \\
\hline 1 & 272428 & 2145 & 2151 & 959 & 483 & 293 & 200 \\
\hline 0.1 & 276244 & 1381 & 1388 & 562 & 273 & 160 & 101 \\
\hline \multicolumn{8}{|c|}{ Data run of $1 / 3 / 13$} \\
\hline 100 & 185999 & 5917 & 25005 & 9634 & 3745 & 1508 & 656 \\
\hline 10 & 262711 & 2322 & 2797 & 1018 & 473 & 262 & 167 \\
\hline 1 & 274132 & 1770 & 1783 & 739 & 403 & 232 & 154 \\
\hline 0.1 & 277525 & 1118 & 1125 & 441 & 231 & 131 & 73 \\
\hline
\end{tabular}




\section{Lead Run}

Lead was measured in the same manner as the polyethylene. The material had dimensions of $30.5 \mathrm{~cm}$ by $30.5 \mathrm{~cm}$ by $30.5 \mathrm{~cm}$ and a total mass as listed in Table 1.1 . The lead was in the form of 26 whole $(5.1 \mathrm{~cm}$ by $10.2 \mathrm{~cm}$ by $20.3 \mathrm{~cm}$ ) bricks, and two half bricks. Figure $10-1$ shows the lead (wrapped in tape) in place on top of the wooden support inside of the multiplicity counter with one side moved.

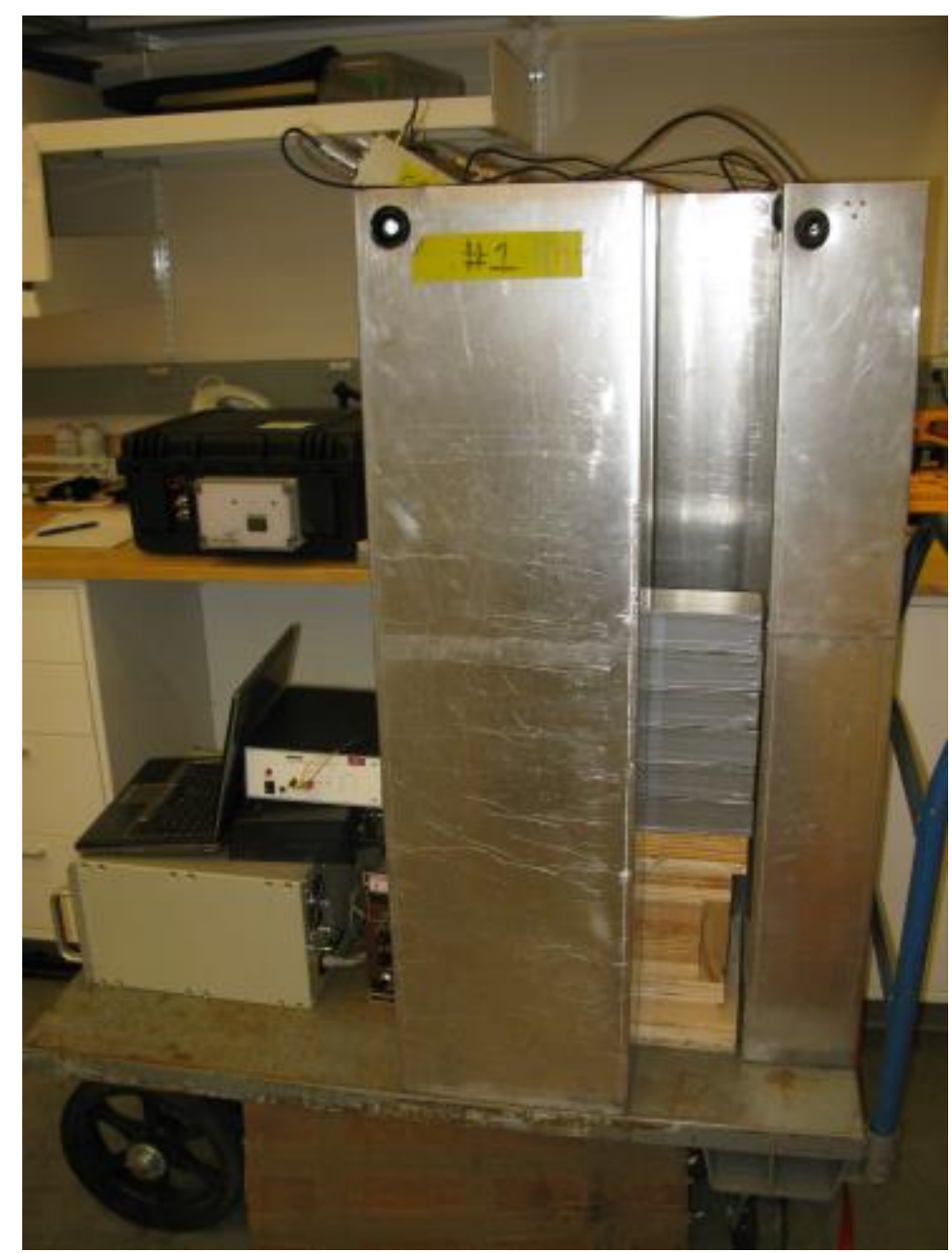

Figure 10-1. Multiplicity counter open showing the lead in position for measurement.

The data in Figure 10-2 show the distribution of neutron multiplicity in cosmic induced events measured by the detector system with a lead target mass. Panel a) shows multiplicity in a $100 \mathrm{~ms}$ time window, panel b) shows multiplicity in a $10 \mathrm{~ms}$ time window, panel c) shows multiplicity in a $1 \mathrm{~ms}$ time window and panel d) shows multiplicity in a $100 \mu$ s time window. The measured data are the black lines, while the red line is a Poisson distribution with the same mean value as the data. It is seen that for all time windows, the multiplicity data deviate from a Poisson distribution since there are a sufficient number of spallation events in lead to be easily observed. 


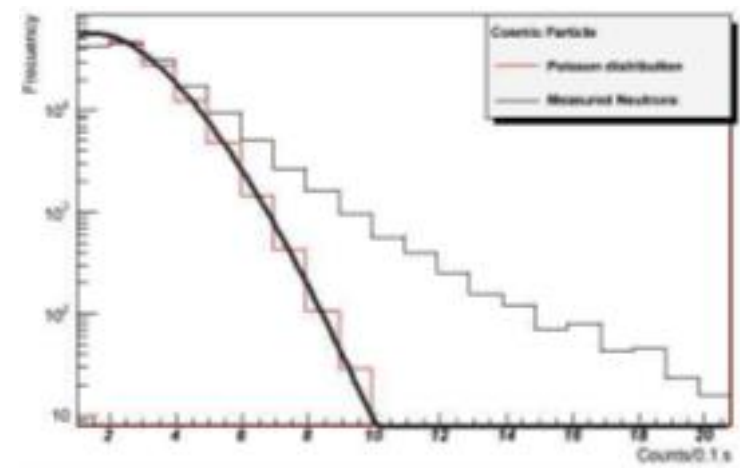

a) $100 \mathrm{~ms}$

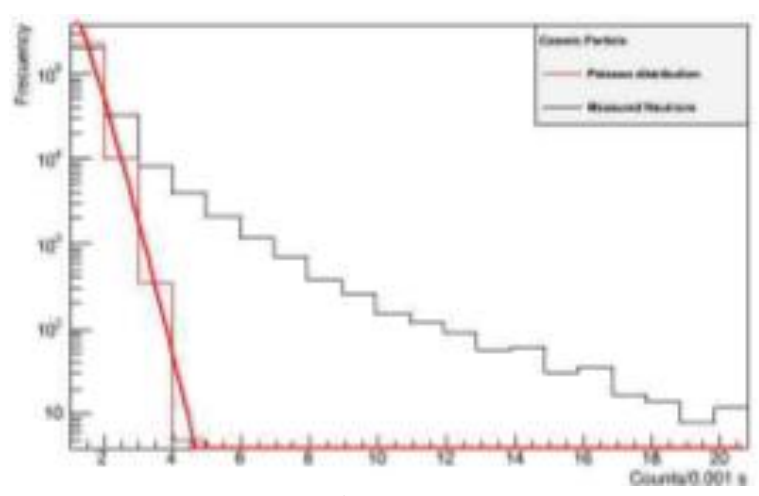

c) $1 \mathrm{~ms}$

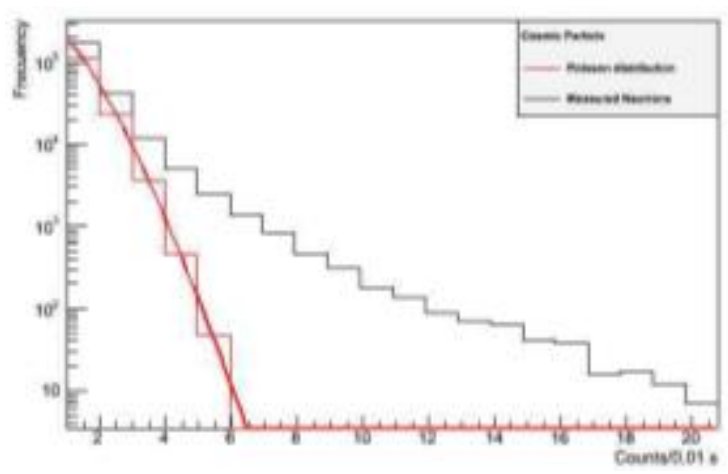

b) $10 \mathrm{~ms}$

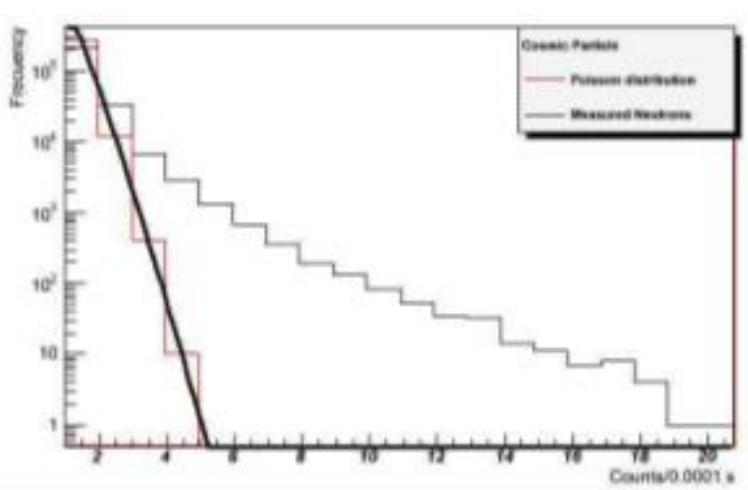

d) $0.1 \mathrm{~ms}$

Figure 10-2. Distribution of neutron multiplicity caused by cosmic neutrons in lead.

The data in Table 10.1 show the results for the measurement of the lead. Considering the $1 \mathrm{~ms}$ time window, the average of 9375 events with a multiplicity of four or more obtained in an average run time of $42,485 \mathrm{~s}$ gives a net rate of $0.221(5) \mathrm{cps}$, using the average background rate and estimating the uncertainty as the standard deviation between measurements.

Table 10.1. Lead run results

\begin{tabular}{|l|l|l|l|l|l|l|l|}
\hline $\begin{array}{l}\text { Gate length } \\
(\mathbf{m s})\end{array}$ & $\begin{array}{l}\text { Total } \\
\text { counts }\end{array}$ & $\begin{array}{l}\text { Counts }> \\
\text { Poisson }\end{array}$ & $\begin{array}{l}\text { Integral } \\
\text { Mult. }>\mathbf{4}\end{array}$ & $\begin{array}{l}\text { Integral } \\
\text { Mult. }>\mathbf{5}\end{array}$ & $\begin{array}{l}\text { Integral } \\
\text { Mult. }>\mathbf{6}\end{array}$ & $\begin{array}{l}\text { Integral } \\
\text { Mult. }>\text { 7 }\end{array}$ & $\begin{array}{l}\text { Integral } \\
\text { Mult. }>8\end{array}$ \\
\hline Data run of 1/7/13 \\
\hline 100 & 157387 & 19724 & 38812 & 21502 & 12093 & 7094 & 4423 \\
\hline 10 & 237207 & 10849 & 11360 & 6285 & 3765 & 2354 & 1508 \\
\hline 1 & 250067 & 9223 & 9229 & 5262 & 3160 & 1980 & 1282 \\
\hline 0.1 & 260991 & 5908 & 5917 & 2979 & 1639 & 942 & 582 \\
\hline Data run of $1 / 8 / 13$ \\
\hline 100
\end{tabular}


Figure 10-3 shows the $1 \mathrm{~ms}$ multiplicity distribution for lead for multiplicity up to 50 . This demonstrates that the multiplicity does eventually go to zero, as would be expected.

\section{Time Correlated Neutron Counter - $1 \mathrm{~ms}$ window}

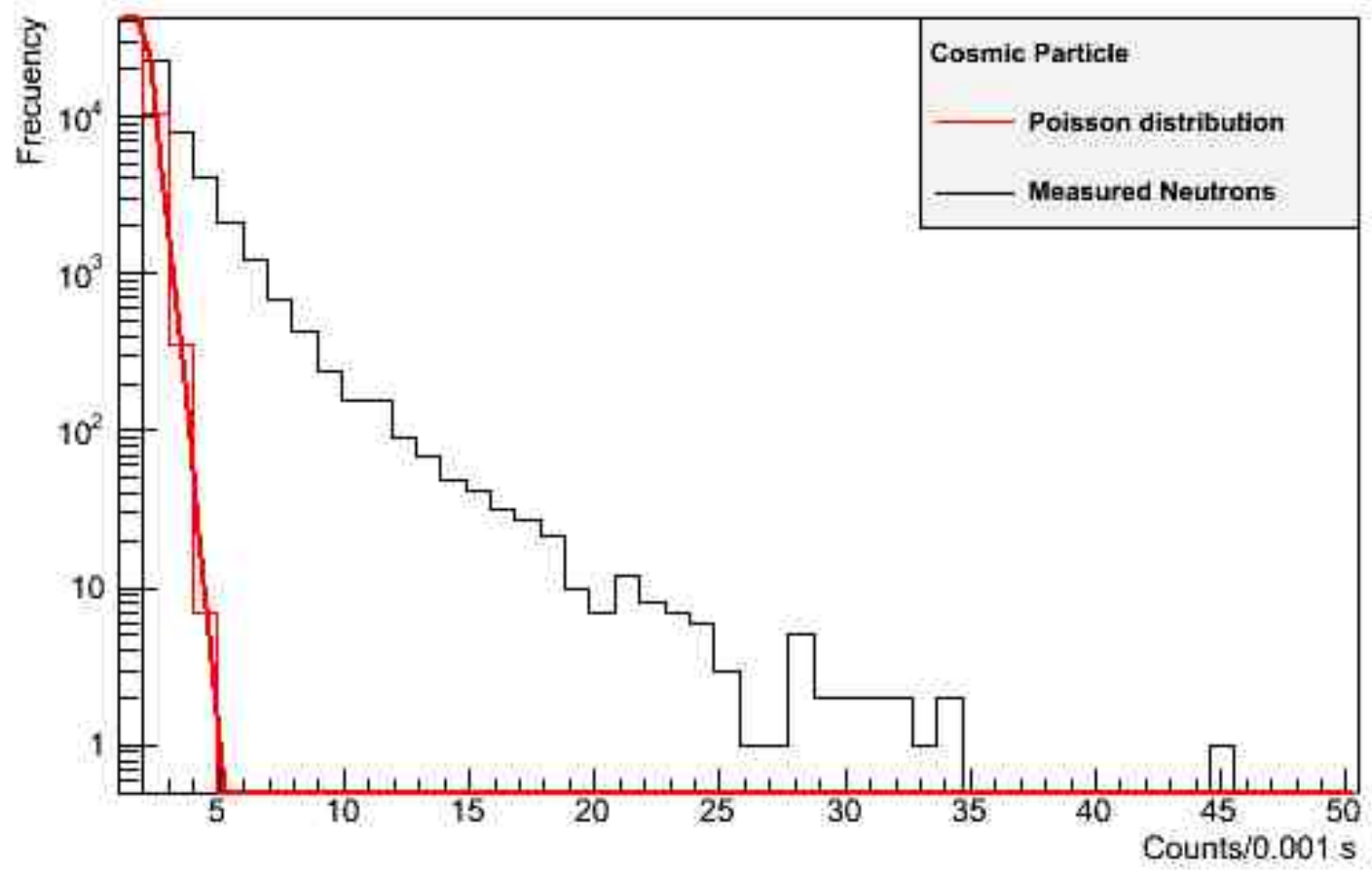

Figure 10-3. Distribution of neutron multiplicity caused by cosmic neutrons in lead up to 50 . 


\section{Tungsten Run}

Tungsten was measured in the same manner as the polyethylene. Because tungsten is expensive, a smaller mass was used and the geometry was different from the other measurements. Based on the measured density, the material tested may be a tungsten alloy ("heavy met") rather than pure tungsten (with a 10\% impact on the measurements). Two different assemblies were available. The first was a cylinder $15 \mathrm{~cm}$ in diameter, and $15 \mathrm{~cm}$ high. The second was a rectangular block, with dimensions $10 \mathrm{~cm}$ by $6.25 \mathrm{~cm}$ by $5 \mathrm{~cm}$. The total mass and volume is listed in Table 1.1. Figure 11-1 shows the tungsten in place on top of the wooden support inside of the multiplicity counter with one side moved.

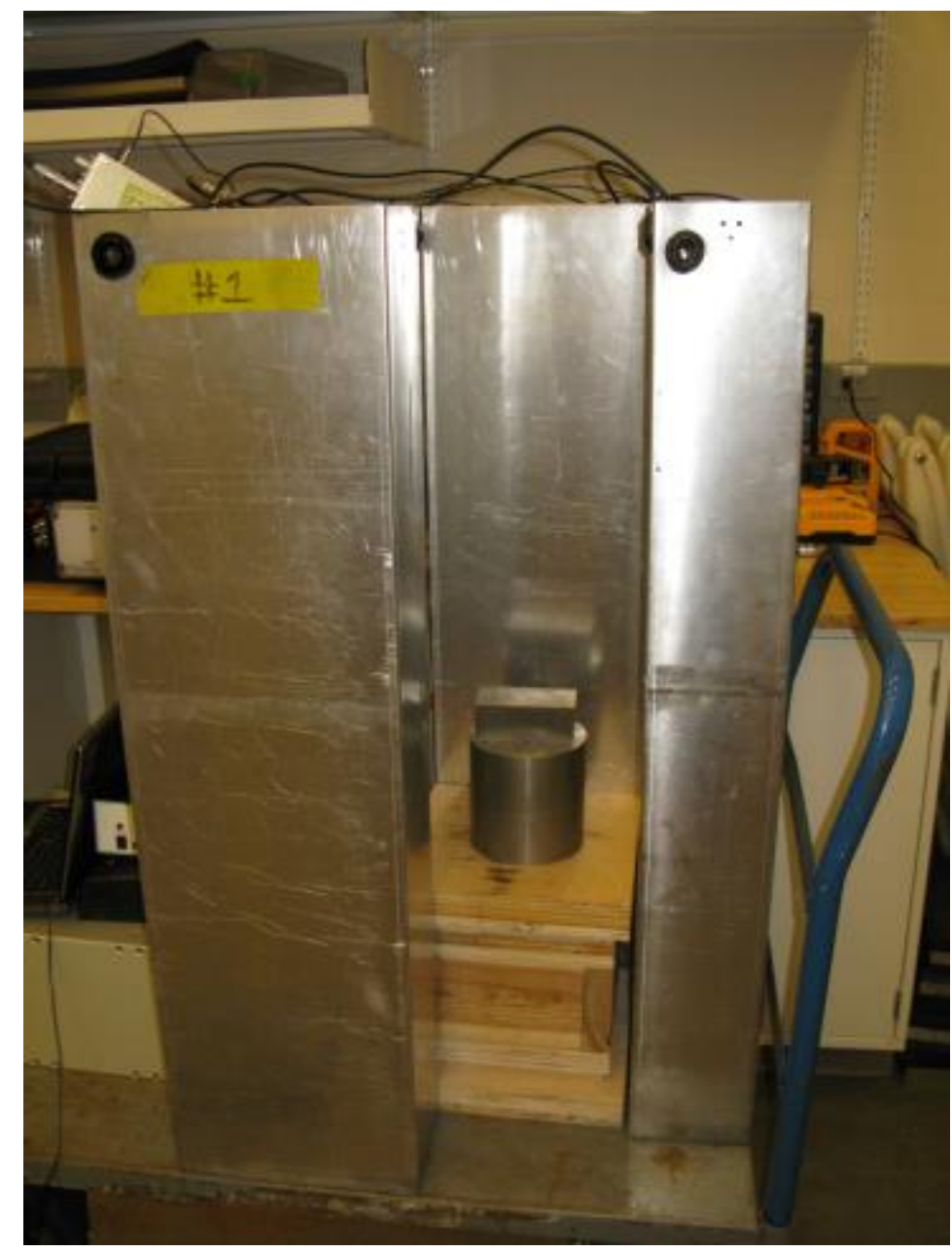

Figure 11-1. Multiplicity counter open showing the tungsten in position for measurement.

The data in Figure 11-2 show the distribution of neutron multiplicity in cosmic induced events measured by the detector system with a tungsten target mass. Panel a) shows multiplicity in a $100 \mathrm{~ms}$ time window, panel b) shows multiplicity in a $10 \mathrm{~ms}$ time window, panel c) shows multiplicity in a $1 \mathrm{~ms}$ time window and panel d) shows multiplicity in a $100 \mu \mathrm{s}$ time window. The measured data are the black lines, while the red line is a Poisson distribution with the same 
mean value as the data. It is seen that for all time windows, the multiplicity data deviate from a Poisson distribution since there are a sufficient number of spallation events in tungsten to be easily observed.

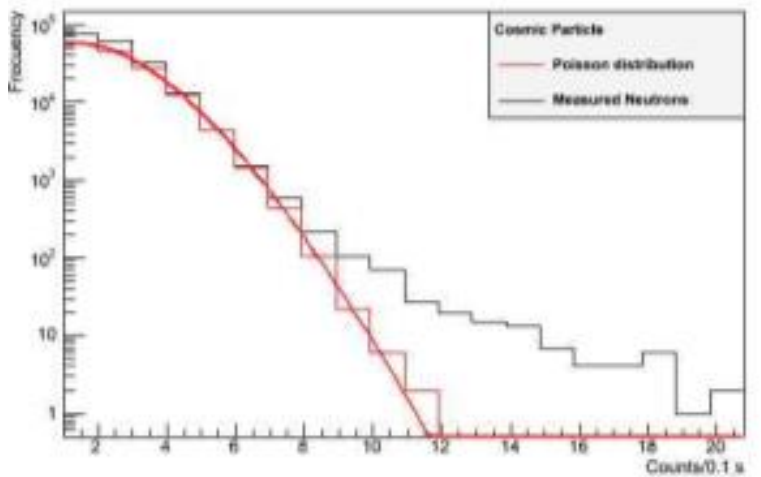

a) $100 \mathrm{~ms}$

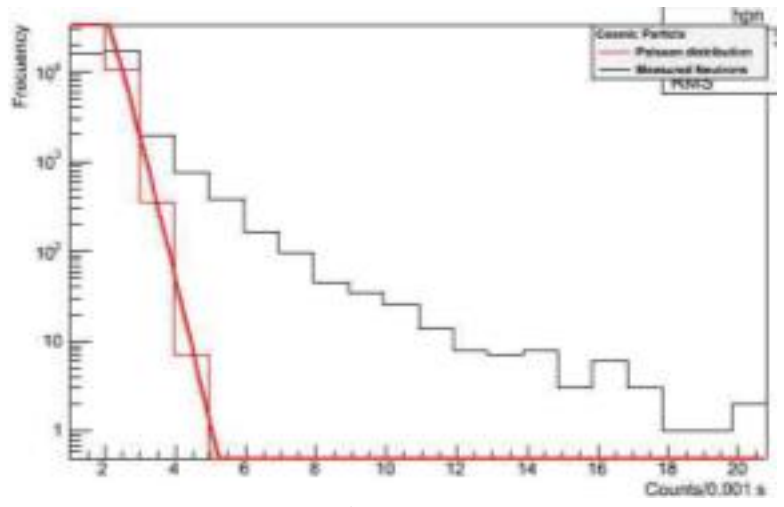

c) $1 \mathrm{~ms}$

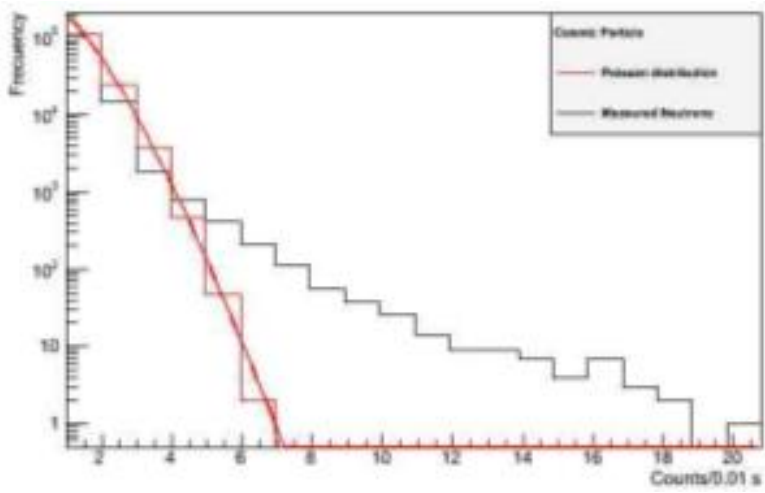

b) $10 \mathrm{~ms}$

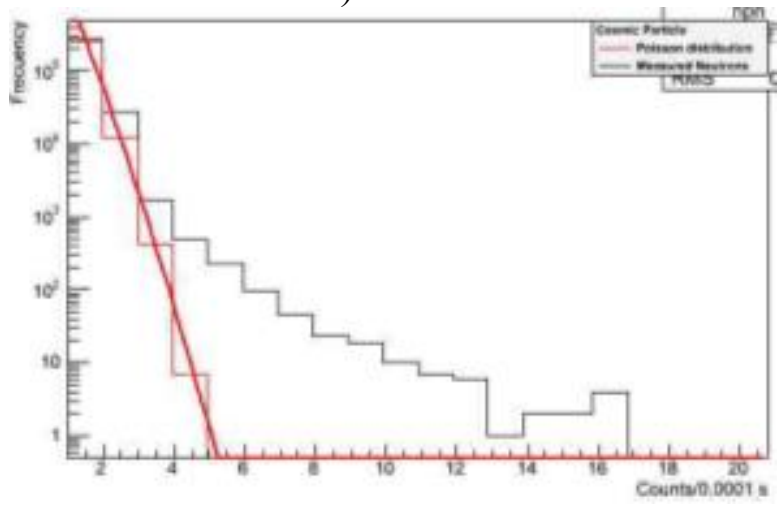

d) $0.1 \mathrm{~ms}$

Figure 11-2. Distribution of neutron multiplicity caused by cosmic neutrons in tungsten.

The data in Table 11.1 show the results for the measurement of the tungsten sample, which was smaller than all the other samples. Considering the $1 \mathrm{~ms}$ time window, the 1575 events with a multiplicity of four or more obtained in a run time of $65,557 \mathrm{~s}$ gives a net rate of $0.023(4) \mathrm{cps}$, using the average background rate and estimating the uncertainty as the same as that seen in the background average. With the assumption that this rate would scale with volume (from 3.11 liter to 28.4 liters, a ratio of 9.1), the rate for a full target volume comparable to the other samples would thus have been $0.21(4)$ cps.

Table 11.1. Tungsten run results $(1 / 11 / 2013)$

\begin{tabular}{|l|l|l|l|l|l|l|l|}
\hline $\begin{array}{l}\text { Gate length } \\
(\mathbf{m s})\end{array}$ & $\begin{array}{l}\text { Total } \\
\text { counts }\end{array}$ & $\begin{array}{l}\text { Counts }> \\
\text { Poisson }\end{array}$ & $\begin{array}{l}\text { Integral } \\
\text { Mult. }>\text { 4 }\end{array}$ & $\begin{array}{l}\text { Integral } \\
\text { Mult. }>\mathbf{5}\end{array}$ & $\begin{array}{l}\text { Integral } \\
\text { Mult. }>\text { 6 }\end{array}$ & $\begin{array}{l}\text { Integral } \\
\text { Mult. }>\text { 7 }\end{array}$ & $\begin{array}{l}\text { Integral } \\
\text { Mult. }>\text { 8 }\end{array}$ \\
\hline 100 & 195261 & 1314 & 20402 & 7195 & 2610 & 1080 & 501 \\
\hline 10 & 266681 & 1709 & 2220 & 970 & 506 & 295 & 181 \\
\hline 1 & 276691 & 1568 & 1575 & 803 & 426 & 261 & 163 \\
\hline 0.1 & 279562 & 924 & 937 & 446 & 215 & 120 & 74 \\
\hline
\end{tabular}


The target materials used for the measurements reported here involved stacks of the same dimensions ( 1 foot cubed) for all except $\mathrm{W}$. The $\mathrm{W}$ target was a smaller volume, and thus the measurement results had to be scaled to the same volume as the other materials. It was assumed that the resulting neutron multiplicity produced scaled linearly with volume. Since the neutron output from a target is a result of a cascade process, the scaling of output might not agree with a linear dependence on volume.

In order to validate the assumption of linear dependence with volume, a series of measurements were made with various volumes of $\mathrm{Pb}$ (length $\mathrm{x}$ depth $\mathrm{x}$ height: 4"x4"x4", 4"x4"x8", 8"x8"x8", 8 8 8 "x12", 8"x12"x12", 12"x12"x12"). Geant4 models of these same dimensions were also run.

Figure 11-3 provides the results from these measurements. The green line in the figure shows what was anticipated as the linear scaling of output with target volume. The purple circles show the results from the model for the appropriate fluence of cosmic neutrons vertically incident on the target, with good agreement with linear scaling with volume. The blue triangles show the experimental measurements for neutron count rate greater than a Poisson background, showing good agreement with the assumption of a linear dependence with volume. The diamonds show the doubles rate (scaled arbitrarily) from a multiplicity analysis of the same data, also agreeing with the volume scaling.

Thus, it is reasonable to use linear volume scaling for the results of the $\mathrm{W}$ target measurements, as was done for the analysis described later in this report.

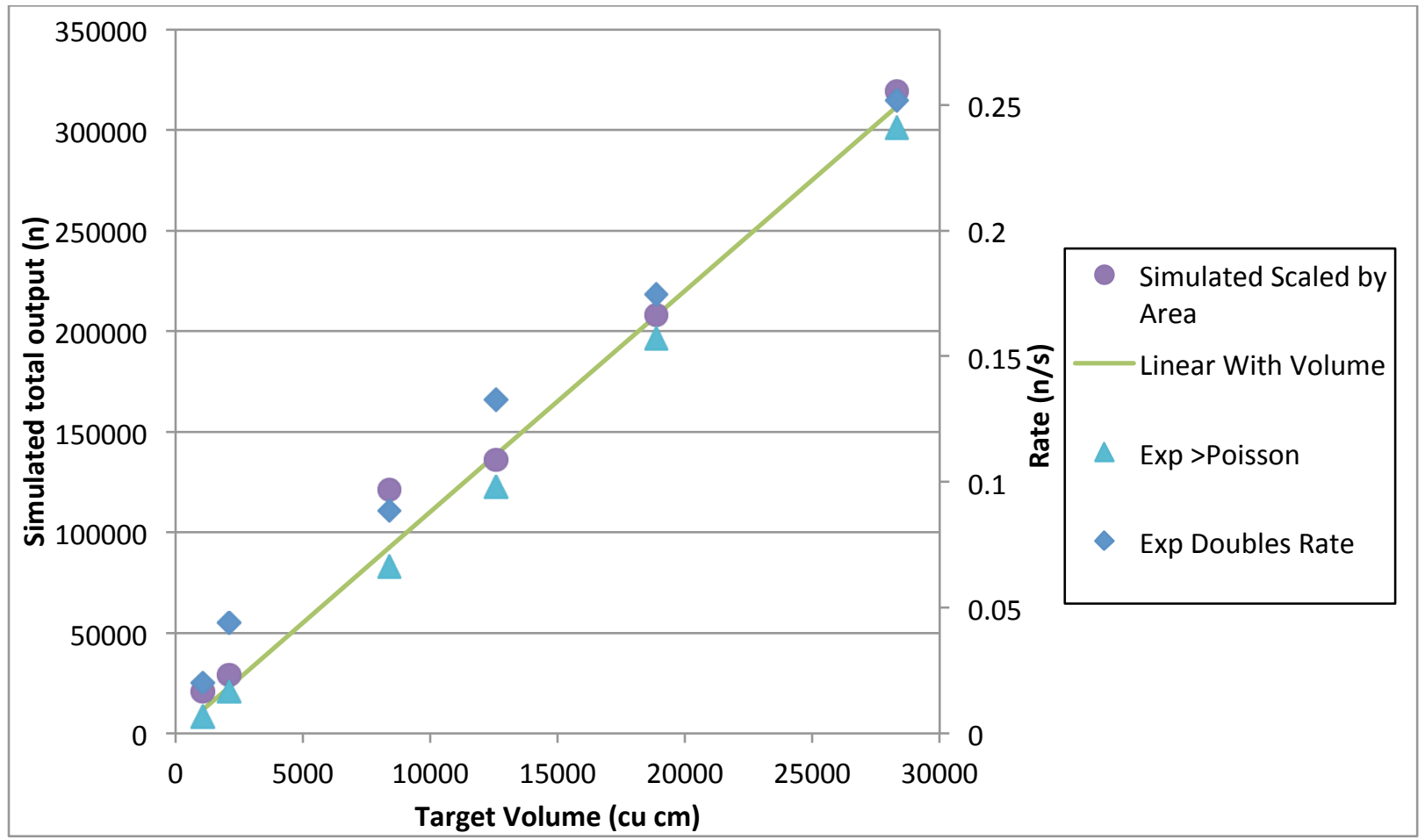

Figure 11-3. Scaling of rate with target volume of $\mathrm{Pb}$. 


\section{Experimental Results}

The data obtained in these measurements can be analyzed in several ways by varying the time window over which events are integrated, and counting multiplicity with varying thresholds.

\subsection{Integrated Experimental Multiplicity Counts}

The data plotted in Figures 12-1 and Figure 12-2 show the experimental total neutron counts versus neutron density obtained from the air, polyethylene, steel, copper, lead, and tungsten samples. The lines are to guide the eye, and the uncertainties are smaller than the markers. The figures differ in the time window used for the integration of counts (10 ms versus $1 \mathrm{~ms})$. The four trends of data in each figure represent counts with multiplicities greater than 5, 6, 7 and 8 . The trend observed in the data is the same for all time windows.

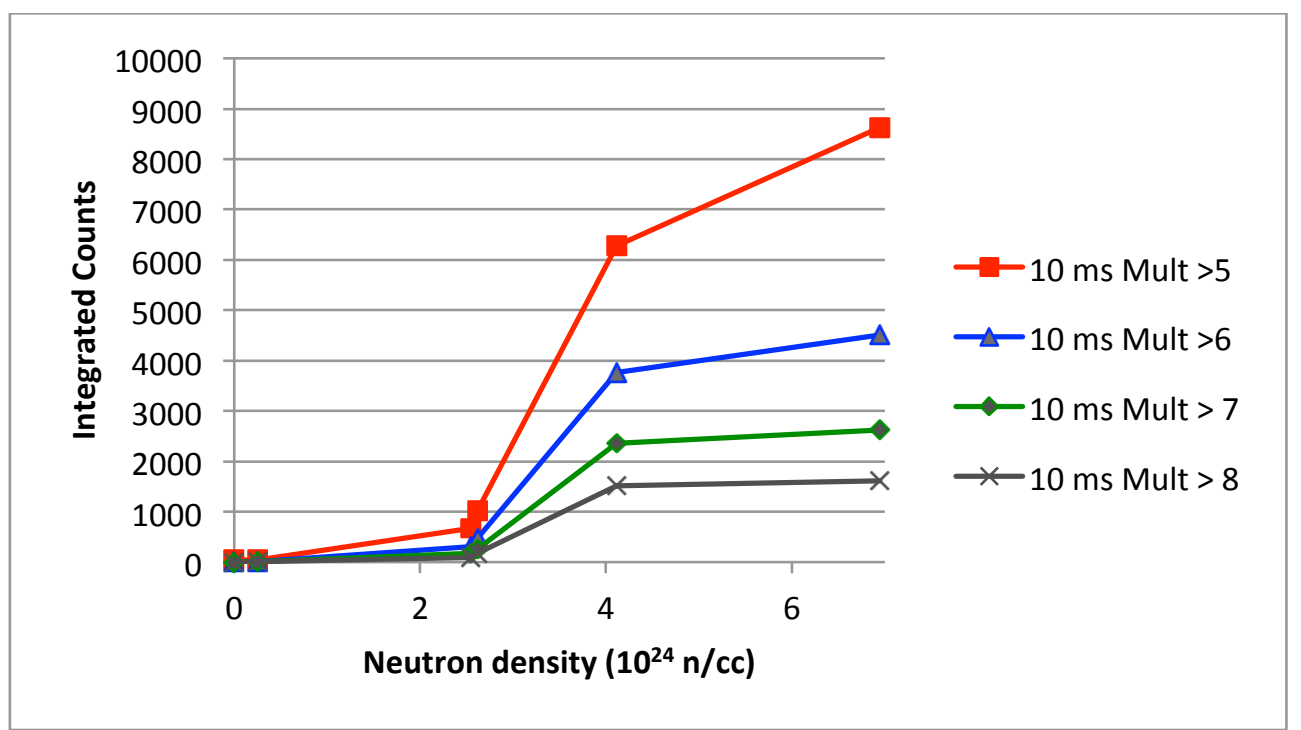

Figure 12-1. Integrated number of events versus neutron density in $10 \mathrm{~ms}$ gate length.

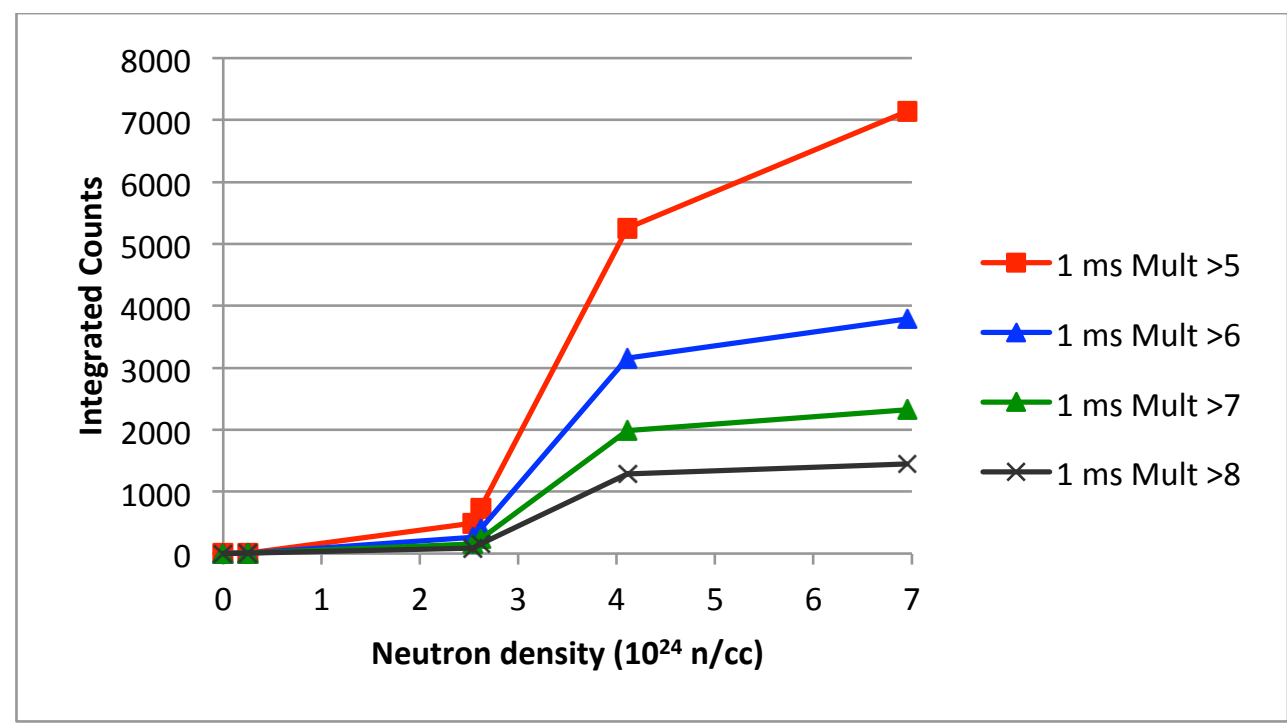

Figure 12-2. Integrated number of events versus neutron density in $1 \mathrm{~ms}$ gate length. 
The tungsten data in the plots has been scaled by volume to be comparable to the other data. As expected, the integrated counts increase with neutron density, but not in a linear fashion. While these plots use neutron density, the same trend is found when using mass density. It is more informative to convert the total counts to a rate so they can be more directly compared.

\subsection{Multiplicity Rate}

One way to count multiplicity is to compare results from the various materials for a selected time window with events counted above a certain multiplicity value. Table 12.1 summarizes the data analysis runs using a $1 \mathrm{~ms}$ time window and a multiplicity value of four or more, six or more, and eight or more, as was presented in each of the earlier sections of this report. Net rates are listed.

The table shows the density and neutron density for each of the materials, and the observed rate of events (in cps) for the fixed volume (one cubic foot) of material used in the measurements, including scaling the tungsten results to this same volume.

Table 12.1. Summary of experimental multiplicity results.

\begin{tabular}{|l|c|c|c|c|c|c|}
\hline Material & $\begin{array}{c}\text { Density } \\
(\mathbf{g} / \mathbf{c c})\end{array}$ & $\begin{array}{c}\text { Target } \\
\text { mass (kg) }\end{array}$ & $\begin{array}{c}\text { Neutron Density } \\
\left(\mathbf{n} / \mathbf{c c} \mathbf{~ x ~ 1 0} \mathbf{~}^{-24}\right)\end{array}$ & $\begin{array}{c}\text { Rate } \\
\text { Mult. }>\text { 4 }\end{array}$ & $\begin{array}{c}\text { Rate } \\
\text { Mult. }>\mathbf{6}\end{array}$ & $\begin{array}{c}\text { Rate } \\
\text { Mult. }>\mathbf{8}\end{array}$ \\
\hline $\mathrm{HDPE}$ & 0.95 & 28.05 & 0.25 & $\begin{array}{c}0.0 \pm \\
0.003\end{array}$ & $\begin{array}{c}0.0 \pm \\
0.0007\end{array}$ & $\begin{array}{c}0.0 \pm \\
0.0004\end{array}$ \\
\hline $\mathrm{Al}$ & 2.7 & 76.8 & 0.84 & $\begin{array}{c}0.0001 \pm \\
0.003\end{array}$ & $\begin{array}{c}0.0001 \pm \\
0.0007\end{array}$ & $\begin{array}{c}0.0 \pm \\
0.0004\end{array}$ \\
\hline $\mathrm{Fe}$ & 7.87 & 222.6 & 2.54 & $\begin{array}{c}0.019 \pm \\
0.003\end{array}$ & $\begin{array}{c}0.0042 \pm \\
0.0007\end{array}$ & $\begin{array}{c}0.0001 \pm \\
0.0004\end{array}$ \\
\hline $\mathrm{Cu}$ & 8.94 & 237.9 & 2.93 & $\begin{array}{c}0.037 \pm \\
0.003\end{array}$ & $\begin{array}{c}0.0081 \pm \\
0.0007\end{array}$ & $\begin{array}{c}0.0029 \pm \\
0.0004\end{array}$ \\
\hline $\mathrm{Pb}$ & 11.3 & 320.1 & 4.11 & $\begin{array}{c}0.220 \pm \\
0.003\end{array}$ & $\begin{array}{c}0.0760 \pm \\
0.0007\end{array}$ & $\begin{array}{c}0.0310 \pm \\
0.0004\end{array}$ \\
\hline $\mathrm{W}$ & 19.3 & 481.9 & 6.95 & $\begin{array}{c}0.210 \pm \\
0.003\end{array}$ & $\begin{array}{c}0.0580 \pm \\
0.0007\end{array}$ & $\begin{array}{c}0.0220 \pm \\
0.0004\end{array}$ \\
\hline
\end{tabular}

This data is plotted in Figure 12-3, showing the event rate in cps versus neutron density for three multiplicity rates (4,6 and 8). The lines are to guide the eye, and the uncertainties are smaller than the markers. The general trend is increasing event rate with neutron density, though the rate for tungsten is comparable to that of lead.

There is uncertainty introduced by the volume-scaling concept used to get the tungsten rate to scale multiplicity data. There are cascade effects inside the target volume that underestimated the rate using a smaller volume of material. A Geant4 simulation was performed of the neutron output from a tungsten sample of the measured size versus a cubic foot of material. The result was a neutron yield increase by a factor of 7.1. This was somewhat smaller than the volume scaling value of 9.1, which may be within the uncertainty of the model, or may be due to attenuation in the material.

These results can be compared with previous ship effect results measured at PNNL, as shown in Figure 12-4 [Kouzes 2008]. The previous measurements had included several low density 
materials, plus iron and lead. While the trend in the new data reported here is the same as seen in the previous measurement, comparing the lead rate and iron rate in the new data to the previous results shows a significant increase in the ratio of these rates. The copper result is also lower than a linear trend would predict. Because the systematic effects are better controlled in the new experiment, these new results are thought to be more representative of the actual effect. The earlier work used a different geometry for each material measured.

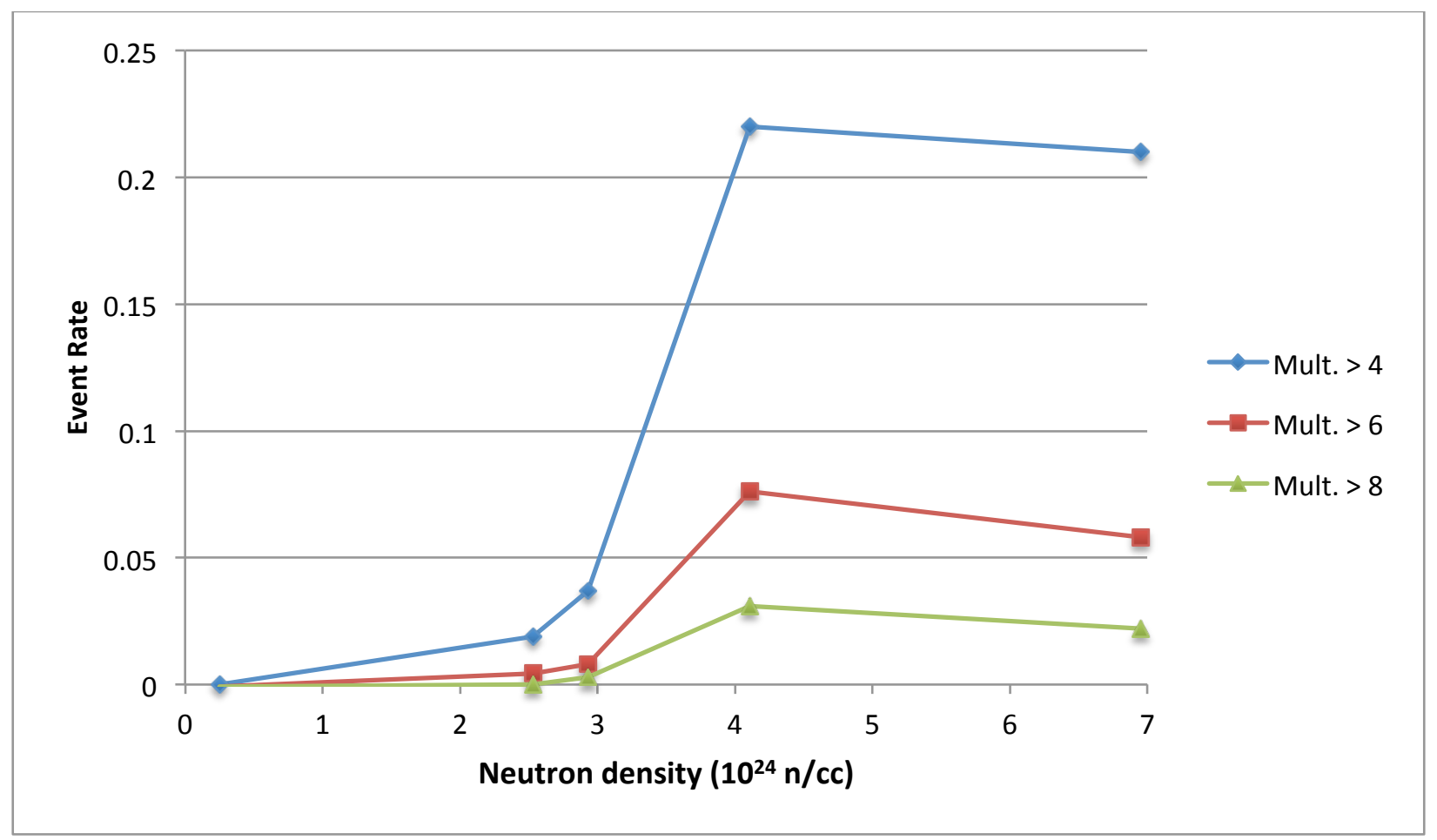

Figure 12-3. Event rate versus neutron density for three multiplicities. 


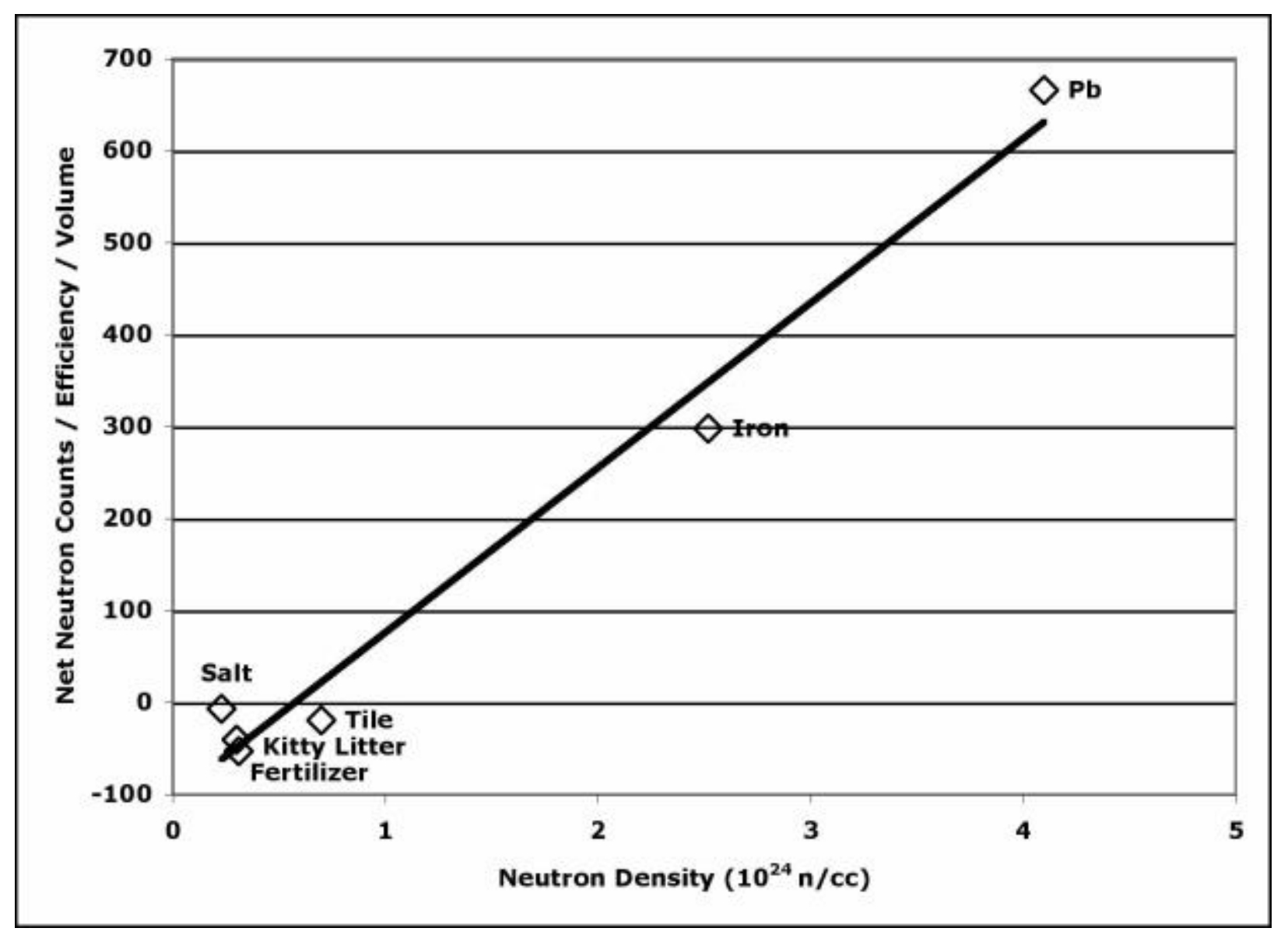

Figure 12-4. Event rate versus neutron density from [Kouzes 2008].

\subsection{Reals and Accidentals Multiplicity Analysis}

There is an alternative method for extracting multiplicity information to that previously discussed. The neutron multiplicity for each run can be extracted from the data by performing an analysis based on the assumption that this data contains a mixture of a "real" correlated distribution of neutrons (R) and the "accidental" (random background) distribution (A). This approach will be referred to as " $\mathrm{R}+\mathrm{A}$ " analysis. Analytical expressions for the factorial moments of the neutron multiplicity distribution were used in this multiplicity analysis. The extracted rates in this analysis are the singles rate $(\mathrm{S}$, multiplicity $=1)$, doubles rate $(\mathrm{D}$, multiplicity $=2)$, triples rate $(\mathrm{T}$, multiplicity $=3)$ and quadruples rate $(\mathrm{Q}$, multiplicity $=4)$, as detailed in [Ensslin 1998]. Multiplicities above four were not computed, since the efficiency of the counter decreases rapidly and this particular data analysis yields null results. In the data presented in this section, the duration of the foreground multiplicity distribution ( $\mathrm{R}+\mathrm{A}$ gate) is $60 \mu \mathrm{s}$, and the background distribution is measured after a delay time interval (A gate) of $4 \mathrm{~ms}$. This analysis method is implemented in commercially available shift registers, such as the Canberra JSR-14. In order to validate the implementation of this analysis method for the use in this work, ${ }^{252} \mathrm{Cf}$ neutron source measurement was made and analyzed using the VME system and a JSR-14. The results are presented in Table 12.2. The agreement in the results indicates the correct implementation of this analysis method. 
Table 12.2. Comparison of multiplicity rates from a JSR14 and the VME system.

\begin{tabular}{|c|c|c|c|}
\hline System & Singles $\left(\mathbf{s}^{-1}\right)$ & Doubles $\left(\mathbf{s}^{-1}\right)$ & Triples $\left.\mathbf{~ s}^{-1}\right)$ \\
\hline JSR-14 & 6422 & 1066 & 109 \\
\hline VME & 6320 & 1310 & 101 \\
\hline
\end{tabular}

Figure 12-5 shows the measured multiplicity computed with the $\mathrm{R}+\mathrm{A}$ method for the different materials studied in this work. A correlation between multiplicities larger than one and the material can be observed.

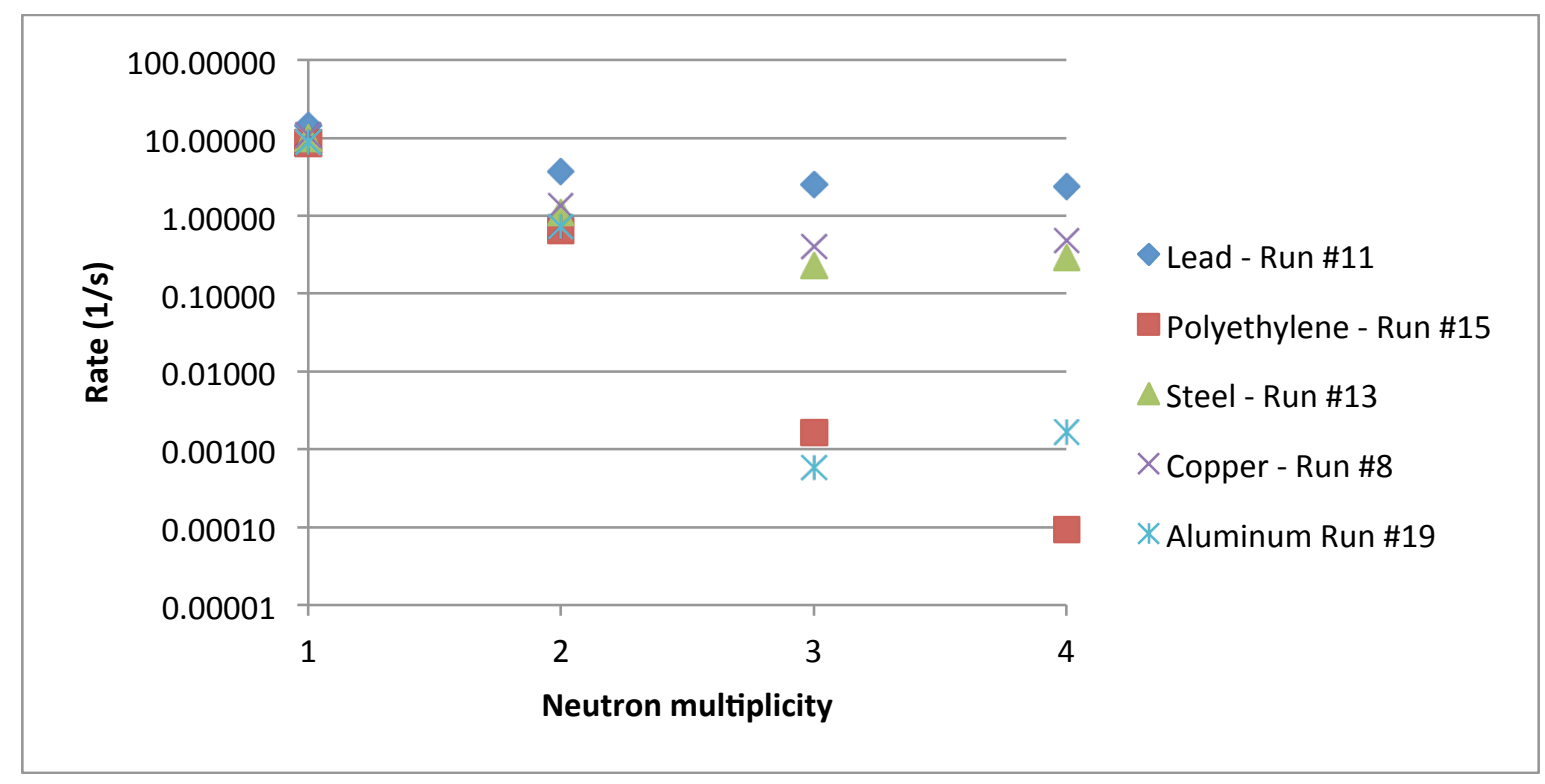

Figure 12-5. Measured neutron multiplicities for the materials studied.

Figure 12-6 shows a comparison of the measured multiplicity found with the R+A method for the materials studied in this work as a function of neutron density. The experimental triples and quads are compared, showing a similar dependence with neutron density. The tungsten data was scaled by volume to be comparable to the other materials. The polyethylene and Al results are consistent with zero. 


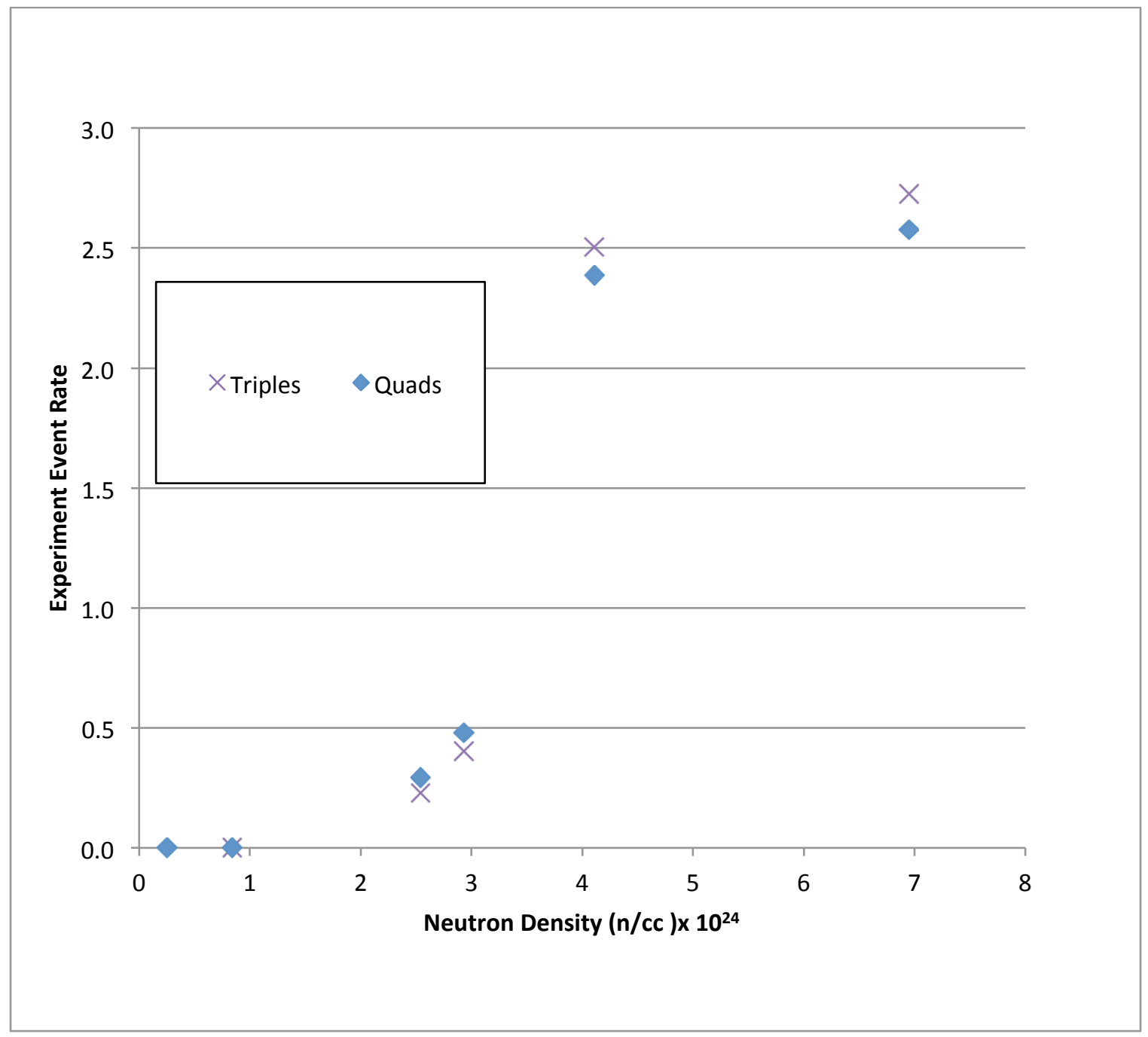

Figure 12-6. Comparison of neutron triples and quads from $\mathrm{R}+\mathrm{A}$ analysis for the materials studied. 


\section{Geant4 Monte Carlo Modeling}

A Monte Carlo model was developed to compare to the measured experimental trend. The simulation is intended to evaluate the neutron physics within the model code and therefore was kept very simple to avoid incorporating other effects, such as neutron detector efficiency, into the simulation. Thus, the model is to predict the relative response of materials, not the absolute response. Monte Carlo modeling was performed using Geant4 [Geant4 2011]. The complete details of the simulation method are given in a previous report [Aguayo 2011]. For the cosmic neutron spectrum at sea level, the model results utilize the Gordon-Goldhagen [Gordon 2004] parameterization of the cosmic ray shower, though a comparison with other spectra was also made.

\subsection{Geant4 Multiplicity Results}

Using a very simplistic geometry consisting of a cubic foot of material, the cosmic neutron spectrum was simulated impinging on the cube from one side only. The output data from the simulation is the total number of secondary neutrons generated from a primary neutron. These secondary neutrons are logged once they leave the cubic foot of volume, so self-attenuation effects are folded into the simulation results. The simulation ran for a total of $10^{5}$ events for each material (2-10 hour simulation runs). This includes neutrons down to $10^{-10} \mathrm{MeV}$ in energy. Figure 13-1 shows the result of the simulation for multiplicity for the incident cosmic neutron spectrum on all the materials measured. The simulation shows neutrons per primary event so no time information is contained in this plot.

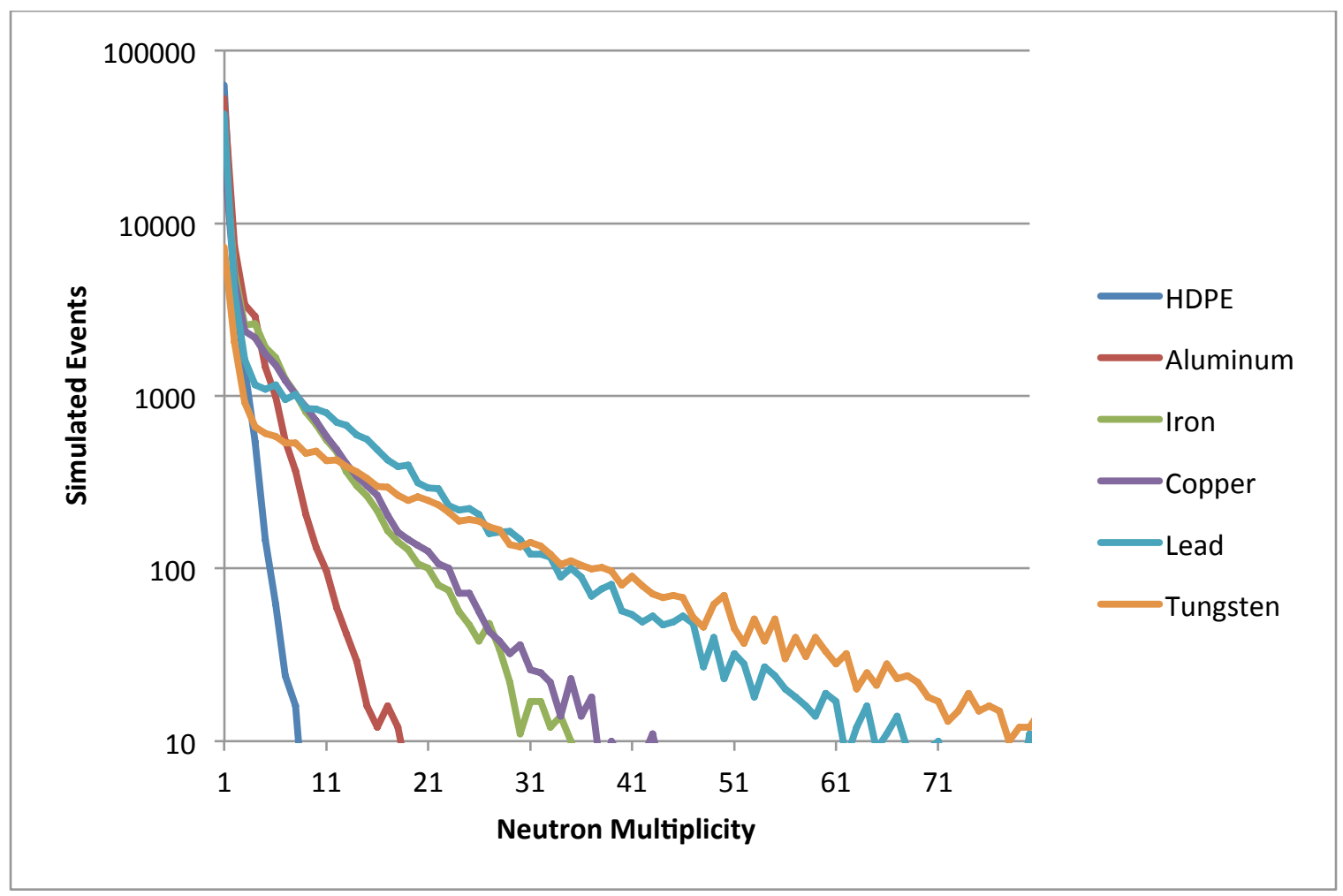


Figure 13-1. Cosmic neutron simulation results for the materials studied.

As can be seen in this graph, which is qualitatively in agreement with the experimental observations from the multiplicity counter, the secondary neutron multiplicity per cosmic event increases as a function of material density, as would be expected.

Figure 13-2 shows similar multiplicity results for some low-Z materials that were computed for comparison. The multiplicity appears to depend strongly on neutron binding energy for these materials.

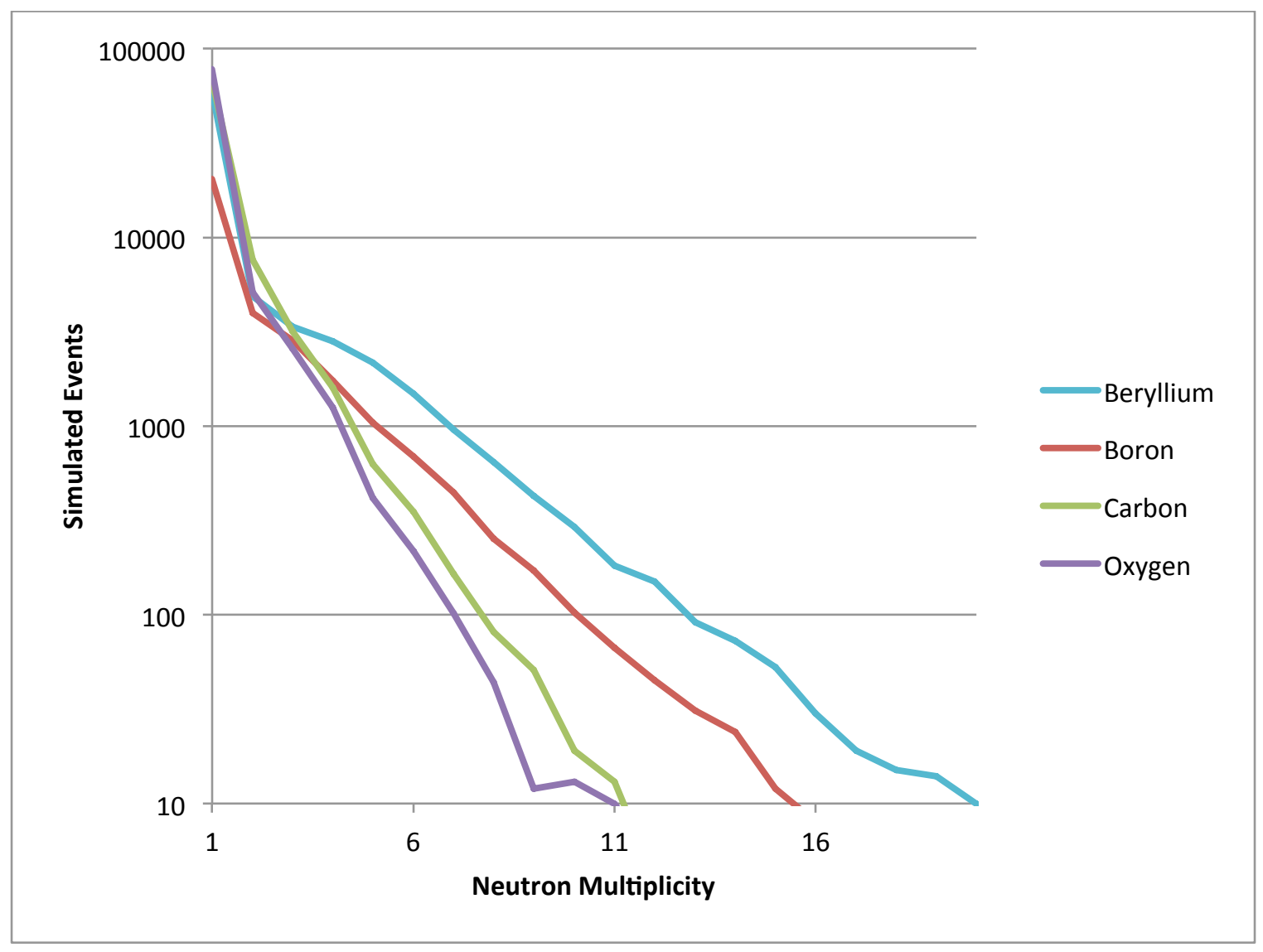

Figure 13-2. Cosmic neutron simulation results for low-Z materials.

Table 13.1 provides the sum of the neutron data represented in these plots. The first row is the sum of all neutrons emitted (for $10^{5}$ incident neutrons. The second row is the ratio of this value normalized to that of $\mathrm{W}$. The third row is the sum of all neutrons for multiplicity greater than four. The fourth row is the ratio of this value normalized to that of $\mathrm{W}$. The fifth row is the ratio of the sum of all neutrons for multiplicity greater than four (row 3 ) to the total number of neutrons (row 1). For Tungsten, 94\% of neutrons emitted have a multiplicity greater than four, while only $2 \%$ of HDPE neutrons have a multiplicity greater than four. 
Table 13.1. Geant4 model results from multiplicity analysis for various materials.

\begin{tabular}{|c|c|c|c|c|c|c|c|c|c|c|}
\hline Material: & Be & B & C & $\mathbf{O}$ & HDPE & Al & $\mathbf{F e}$ & $\mathrm{Cu}$ & $\mathbf{P b}$ & W \\
\hline $\begin{array}{l}\text { Total } \\
\text { neutrons: }\end{array}$ & 63431 & 138009 & 107551 & 105838 & 79721 & 116780 & 163938 & 169427 & 317447 & 268510 \\
\hline $\begin{array}{l}\text { Total neutrons } \\
\text { ratio to } W \text { : }\end{array}$ & 0.24 & 0.51 & 0.40 & 0.39 & 0.30 & 0.43 & 0.61 & 0.63 & 1.18 & 1.00 \\
\hline $\begin{array}{l}\text { Total neutrons } \\
\text { Mult. > 4: }\end{array}$ & 19686 & 47068 & 7992 & 4869 & 1477 & 27171 & 106847 & 119005 & 256920 & 251722 \\
\hline $\begin{array}{l}\text { Total neutrons } \\
\text { Mult. > } 4 \text { ratio } \\
\text { to } W \text { : }\end{array}$ & 0.08 & 0.19 & 0.03 & 0.02 & 0.01 & 0.11 & 0.42 & 0.47 & 1.02 & 1.00 \\
\hline $\begin{array}{l}\text { Ratio Mult. }>4 \\
\text { to total: }\end{array}$ & 0.31 & 0.34 & 0.07 & 0.05 & 0.02 & 0.23 & 0.65 & 0.70 & 0.81 & 0.94 \\
\hline
\end{tabular}

\subsection{Geant4 Monoenergetic Neutrons}

In addition to the neutron parameterization, simulations were run for $10^{6}$ monoenergetic neutrons at $1,10,100$, and $1000 \mathrm{MeV}$ vertically into a cubic foot of $\mathrm{Pb}$, and the number of outgoing neutrons was counted (including non-interacting neutrons). The results were $0.8,1.5,7.2$, and 30 outgoing neutrons per incident neutron, respectively. These are compared to the MCNP results provided in the next Section.

\subsection{Geant4 Total Neutron Production}

The simulation results for total outgoing neutrons for each material for $10^{5}$ incoming neutrons with a cosmic ray distribution are shown as a function of material neutron density in Figure 13-3 (Figure 13-4 is the same data with an expanded vertical scale not showing $U$ ). This includes neutrons down to $10^{-10} \mathrm{MeV}$ in energy.

The materials with blue markers are the ones that were also measured, while the red markers are computed results for other possible materials. Hydrogenous and low Z materials (water, polyethylene) fall in the lower left corner of the plot, representing the lowest neutron yield, whereas lead and tungsten show the maximum yield of neutrons from the materials studied.

The models predict a generally linear dependence of neutron yield with neutron density, as indicated by the trend line (limited to those materials that were measured). Uranium is an outlier and unfortunately was not measured (the measurement is complicated by the spontaneous neutrons emitted by $\mathrm{U}$, and would require a measurement underground to determine the spontaneous component). Further investigation is required to understand the physics of this trend. Such factors as the dependence on nuclear shell structures in the model have not been studied. Similar trends are seen for multiplicities greater than 6 and 8, so the specific analysis method is not crucial to the trend that is observed in the modeling results.

Table 13.2 provides the data for these plots. The last column of the table provides the expected neutron emission rate from the target materials in neutrons per second based on $134 \mathrm{~m}^{-2} \mathrm{~s}^{-1}$ [Gordon 2004] incident cosmic ray neutrons assumed to hit the one square foot top of the target sample. These numbers do not include the detector efficiency value $(\sim 17 \%$ for a fission 
spectrum), which would give an expected detection rate from one to seven per second for the experiment, depending on the target material.

Table 13.2. Model results for total outgoing neutrons for materials for $10^{5}$ incoming neutrons.

\begin{tabular}{|l|r|r|r|}
\hline Material & $\begin{array}{c}\text { Neutron } \\
\text { Density }\end{array}$ & $\begin{array}{c}\text { Total Outgoing } \\
\text { Neutrons }\end{array}$ & $\begin{array}{c}\text { Expected } \\
\text { Outgoing Rate } \\
\text { (n/s) }\end{array}$ \\
\hline HDPE & 0.25 & 79743 & 10 \\
\hline Al & 0.84 & 118082 & 15 \\
\hline Fe & 2.56 & 168071 & 21 \\
\hline Cu & 3.22 & 178153 & 22 \\
\hline Pb & 4.12 & 332253 & 41 \\
\hline W & 6.52 & 297540 & 37 \\
\hline & & & 10 \\
\hline H2O & 0.27 & 83422 & 12 \\
\hline K & 0.27 & 98375 & 13 \\
\hline Concrete & 0.69 & 104860 & 23 \\
\hline Ba & 1.25 & 185491 & 31 \\
\hline Sn & 2.55 & 245069 & 25 \\
\hline Mo & 3.46 & 202295 & 38 \\
\hline Bi & 3.54 & 305922 & 120 \\
\hline Hg & 4.91 & 313725 & \\
\hline U & 6.98 & 965897 & \\
\hline
\end{tabular}

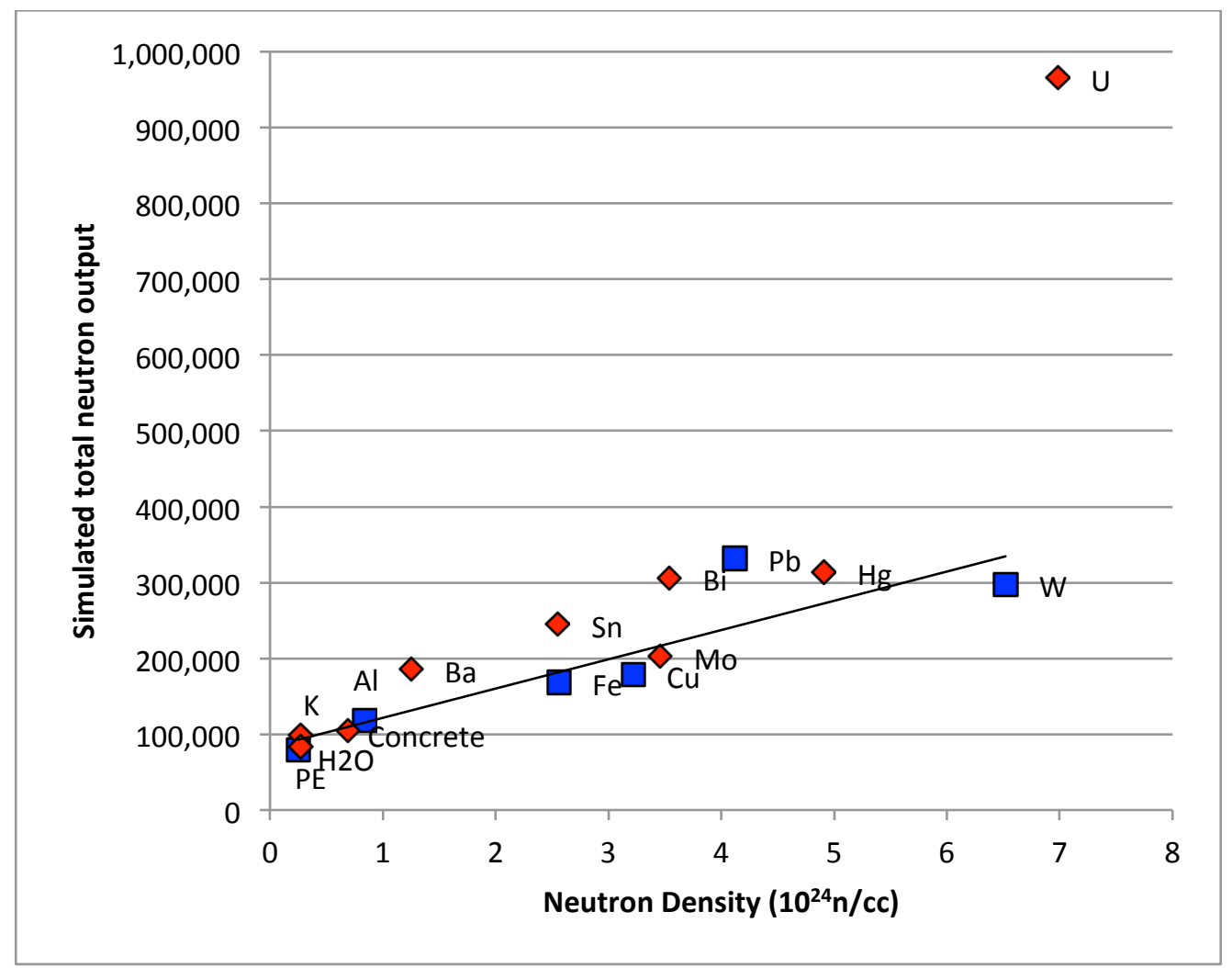

Figure 13-3. Simulated neutrons out from a cubic foot of material as a function of neutron density. 


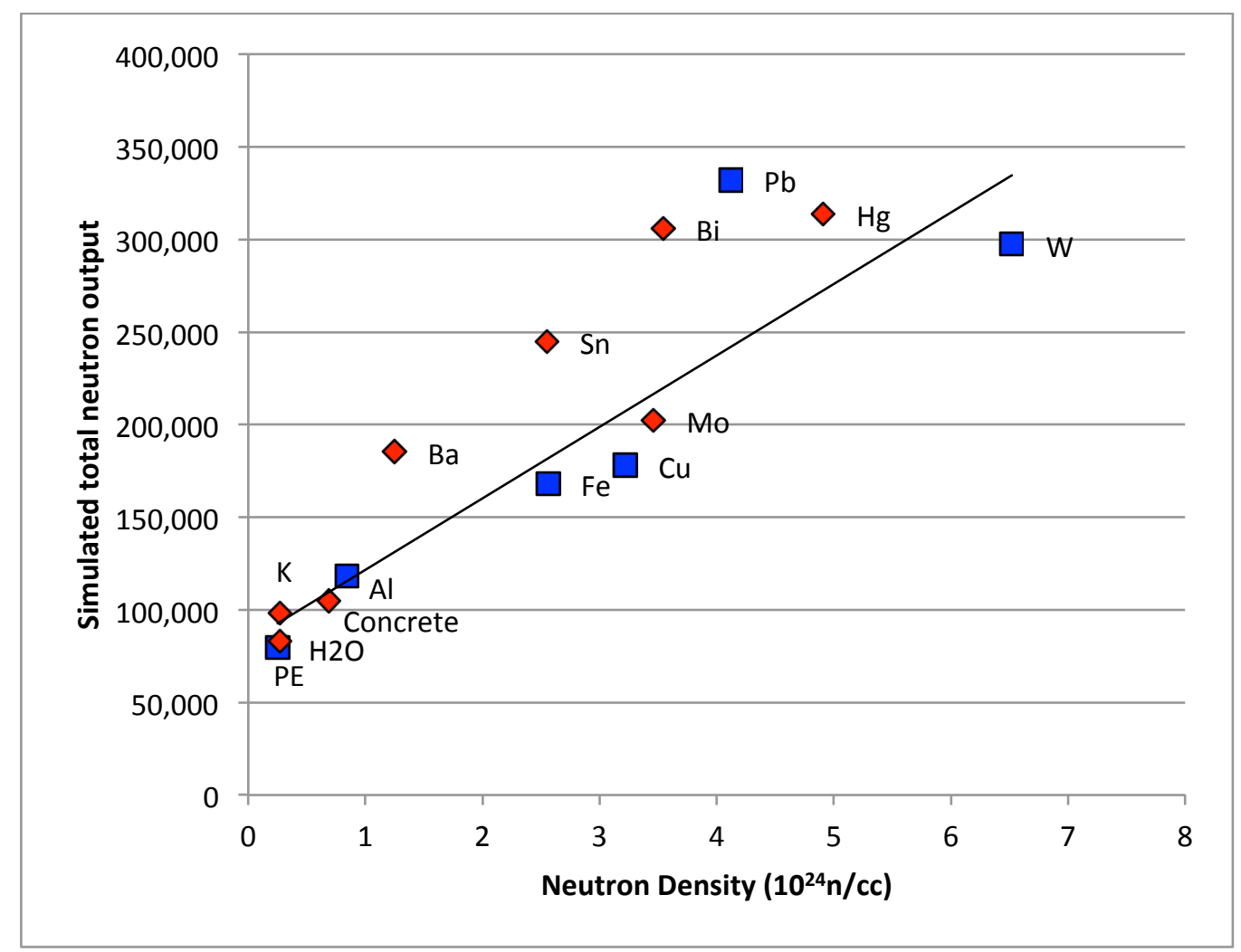

Figure 13-4. Simulated neutrons out from a cubic foot of material as a function of neutron density.

\subsection{Geant4 Outgoing Neutron Energy Spectra}

The computer model only goes as far as determining the neutron flux leaving the cube of material and ignores any subsequent interactions. The coincidence counter efficiency depends on the impinging neutron spectrum. One effect that is not taken into account is that the energy spectrum coming out of the different materials varies in ways that results in a different response from the coincidence counter. Figure 13-5 shows the computed spectra for neutrons exiting each of the materials. The detector is mostly sensitive to neutrons below a few $\mathrm{MeV}$ (as seen in Figure 2-4), where these spectra are fairly similar in shape. 


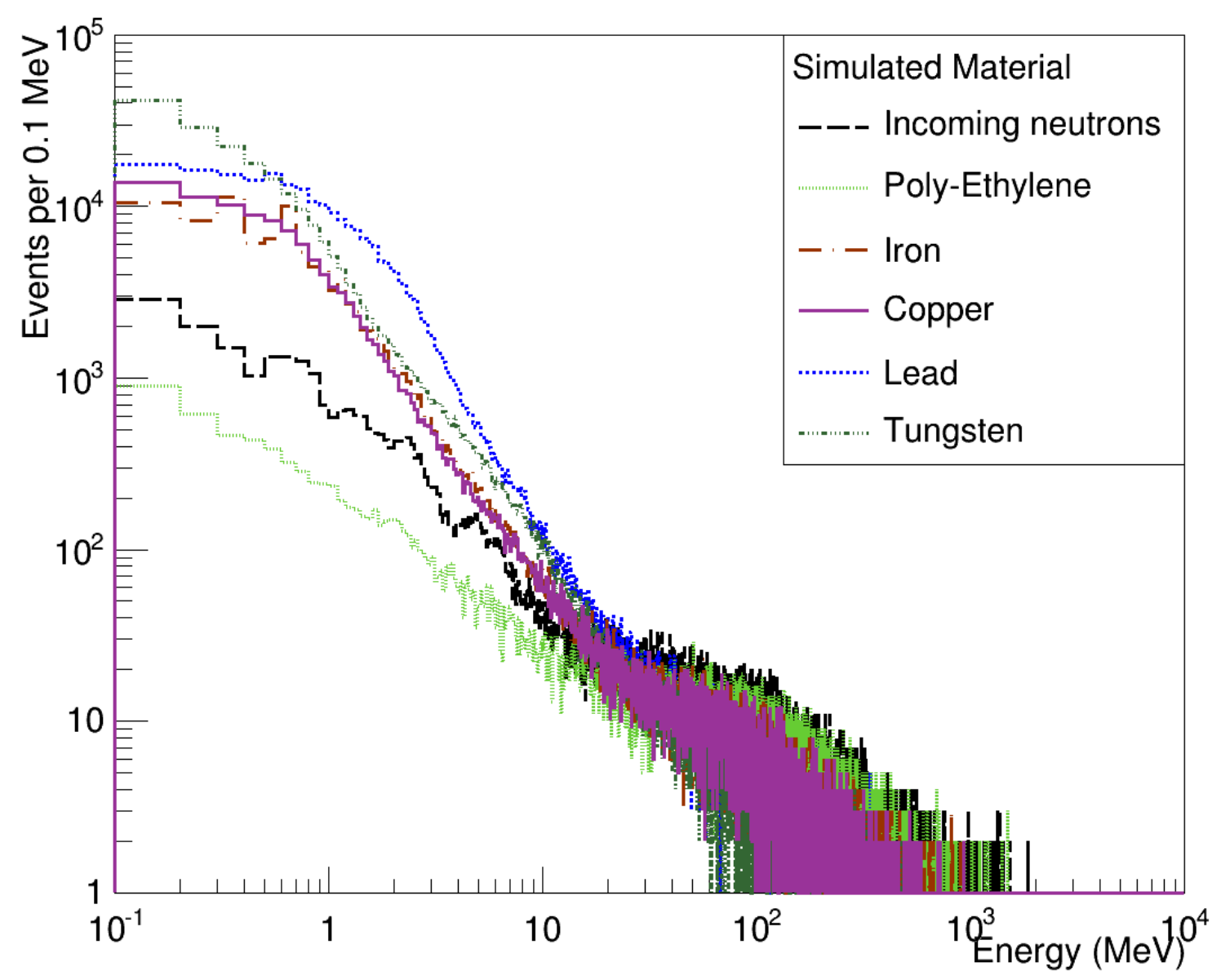

Figure 13-5. Spectra computed with Geant4 for neutrons exiting each of the materials.

\subsection{Geant4 Incoming Cosmic Neutron Energy Spectra}

As was shown in Figure 1-1, there are some differences in the shape of the incoming neutron spectrum at the Earth's surface from different measurements and models. The Geant4 model was run for the $\mathrm{Cu}$ and $\mathrm{Pb}$ targets for three different neutron spectra to see to what degree these differences affect the results. Table 13.2 shows the ratio of the $\mathrm{Cu}$ to $\mathrm{Pb}$ outgoing neutron values for these three different cosmic neutron spectra.

Table 13.3. Ratio of outgoing neutrons for $\mathrm{Cu} / \mathrm{Pb}$ for three spectra.

\begin{tabular}{|l|c|}
\hline \multicolumn{1}{|c|}{ Neutron Spectrum } & Ratio of Outgoing Neutrons $\mathbf{C u} / \mathbf{P b}$ \\
\hline CRY Model & 0.66 \\
\hline Ziegler & 0.77 \\
\hline Goldhagen & 0.54 \\
\hline
\end{tabular}

This result indicates that the choice of neutron parameterization could be responsible for $\sim 25 \%$ of the difference in the comparison between model and experiment, based on the variation seen in the ratio of $\mathrm{Cu}$ to $\mathrm{Pb}$. This is one of the contributing factors to observed differences between models, and between models and experiment, though model predictions to this accuracy are adequate for shielding designs. 


\section{MCNPX Monte Carlo Model Comparisons}

As a comparison to the Geant 4 simulations, a model was created using version 2.70 of MCNPX [Pelowitz 2011] of the cubes of materials, and responses were calculated to cosmic neutrons, protons and muons. In each case, a $30.8 \mathrm{~cm}$ x $30.8 \mathrm{~cm}$ square downward-directed "beam" of particles was incident on the cube of material, and the neutrons leaving all sides of the cube were tallied.

The Monte-Carlo code MCNPX was developed to extend the neutron-photon-electron transport capabilities of MCNP [MCNP] to radiation transport applications for all particles and all energies. This extended capability was accomplished by providing a large number of physics models for use above the $(20 \sim 150 \mathrm{MeV})$ limits in the tabulated cross-section data. One highenergy application for which the physics models were developed in MCNPX was for the design of accelerator spallation targets, and those physics models were used for the MCNPX calculations reported here.

In MCNPX, the calculation of nuclear spallation above the tabulated cross-section data limits is performed in three stages: intra-nuclear cascade (INC), evaporation, and gamma decay. The INC stage involves elastic and non-elastic nuclear scattering and, if at high-enough energies, their emission of high-energy particles (e.g., pions) and light ions (e.g., deuterons). At lower energies the INC stage uses a pre-equilibrium model for the transition from the first reaction to "equilibrium," where all particles are below a threshold for direct particle production. Then the residual nuclei "evaporate" neutrons, protons, light ions, via fission, etc., followed the last stage for decay of gamma rays.

The evaluation in MCNPX of the above processes is done via the Los Alamos High-Energy Transport module, LAHET, [Prael 1989; Prael 1994]. For the targets and energies evaluated in this study, $10^{6}$ source neutrons were used, and neutrons, protons, and charged pions were the primary particles transported ("tracked"). The physics model used was the (default) Bertini INC model as implemented in the LAHET module. For more details of that model see the LAHET publications and other references given in the MCPNX user manual [Pelowitz 2011].

\subsection{MCNP Monoenergetic Neutron Results}

The MCNP simulations were run for monoenergetic neutrons ( $1 \mathrm{MeV}, 10 \mathrm{MeV}, 100 \mathrm{MeV}$, and 1 $\mathrm{GeV}$ ) incident on cubes of various materials. Figure 14-1 shows the number of neutrons out per incident neutron for various materials at several energies. The resulting spectra in Figures 14-2 and 14-3 show the results for outgoing neutrons include full energy (unscattered) neutrons plus lower energy neutrons from scattering and spallation events. There is a broad structure around one $\mathrm{MeV}$ in each case. Except for the polyethylene spectra that drop off more toward low energy, the spectra are very similar for all materials. The drop off at low energy for polyethylene may just be due to absorption of neutrons in this hydrogenous material.

Table 14.1 provides the ratio of outgoing neutrons to incoming neutrons for each of these monoenergetic beams for the $\mathrm{Pb}$ cube. It is seen that the lowest energy $(1 \mathrm{MeV})$ neutrons are scattered, but produce no net increase in neutrons, as expected due to the reaction energies required to produce secondary reactions. At $10 \mathrm{MeV}$, there are a few net excess neutrons, likely due to elastic knockout scattering. The $100 \mathrm{MeV}$ and $1 \mathrm{GeV}$ results show an increasing cascade process occurring. At $1 \mathrm{GeV}$, about 27 neutrons are produced on average for every incoming 
neutron. The cosmic ray spectrum flux (discussed below) produces 2.4 neutrons per incident neutron.

The table also shows a comparison to the Geant4 results discussed in the previous Section. It is seen that the MCNPX and Geant 4 results show some variance (from $7 \%$ to $27 \%$ difference) for the monoenergetic results. This result provides some confidence that the two modeling approaches are giving fairly consistent results.

Table 14.1. Ratio of outgoing neutrons to incoming neutrons for $\mathrm{Pb}$ cube.

\begin{tabular}{|c|c|c|c|}
\hline $\begin{array}{c}\text { Neutron } \\
\text { Energy (MeV) }\end{array}$ & $\begin{array}{c}\text { MCNPX Ratio of } \\
\text { Outgoing Neutrons to } \\
\text { Incoming Neutrons }\end{array}$ & $\begin{array}{c}\text { Geant4 Ratio of } \\
\text { Outgoing Neutrons to } \\
\text { Incoming Neutrons }\end{array}$ & $\begin{array}{c}\text { Percent } \\
\text { Difference } \\
\text { Geant4-MCNP }\end{array}$ \\
\hline Cosmic Flux & 2.4 & 3.3 & $27 \%$ \\
\hline 1 & 1.0 & 0.8 & $-25 \%$ \\
\hline 10 & 1.4 & 1.5 & $7 \%$ \\
\hline 50 & 3.8 & - & - \\
\hline 100 & 5.6 & 7.2 & $22 \%$ \\
\hline 200 & 8.7 & - & - \\
\hline 600 & 13 & - & - \\
\hline 800 & 22 & - & $10 \%$ \\
\hline 1000 & 27 & 30 & - \\
\hline 1200 & 30 & - & \\
\hline
\end{tabular}

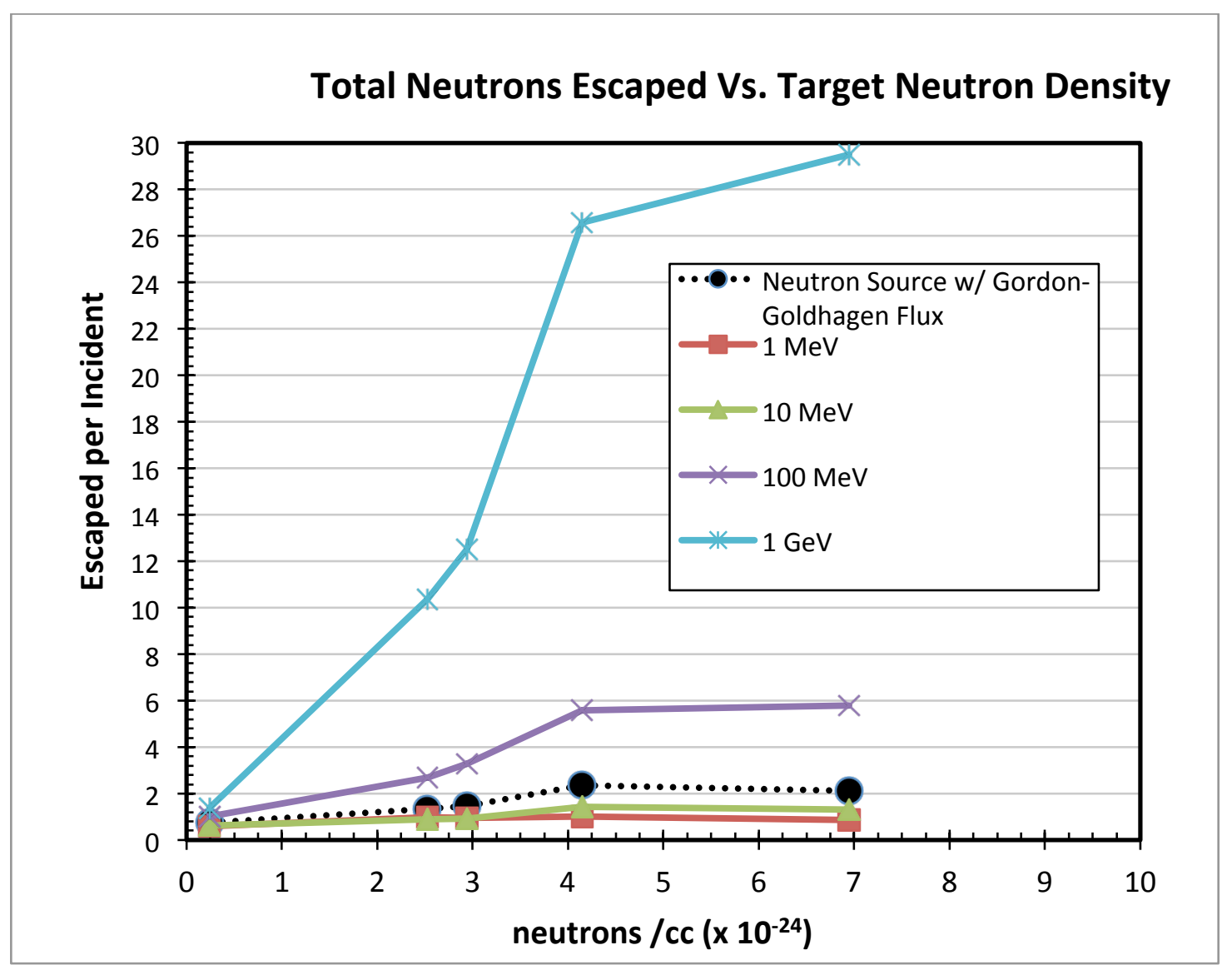

Figure 14-1. MCNP results for total neutrons out at several energies for various materials. 


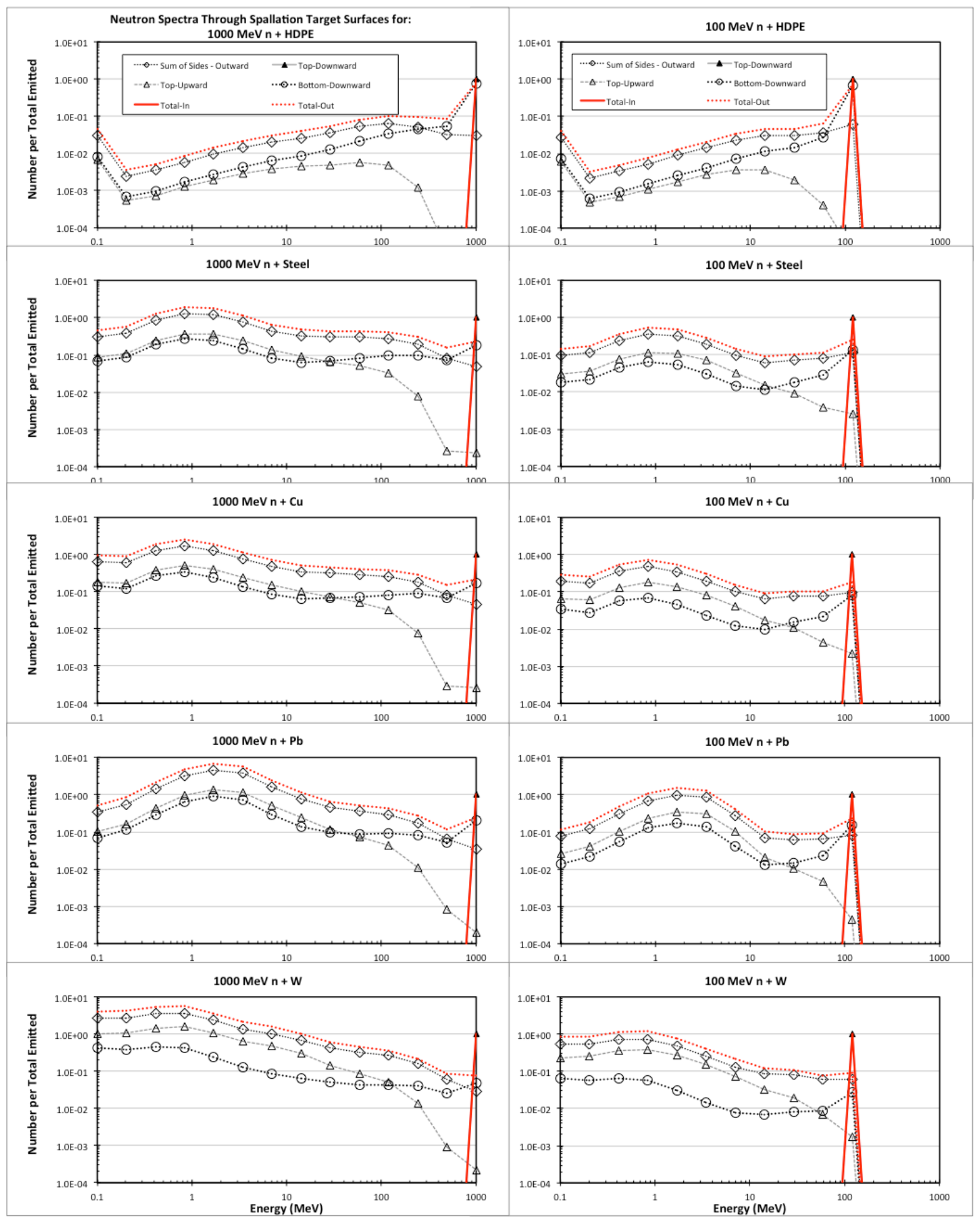

Figure 14-2. MCNP results for $1 \mathrm{GeV}$ and $100 \mathrm{MeV}$ monoenergetic neutrons on various materials. 


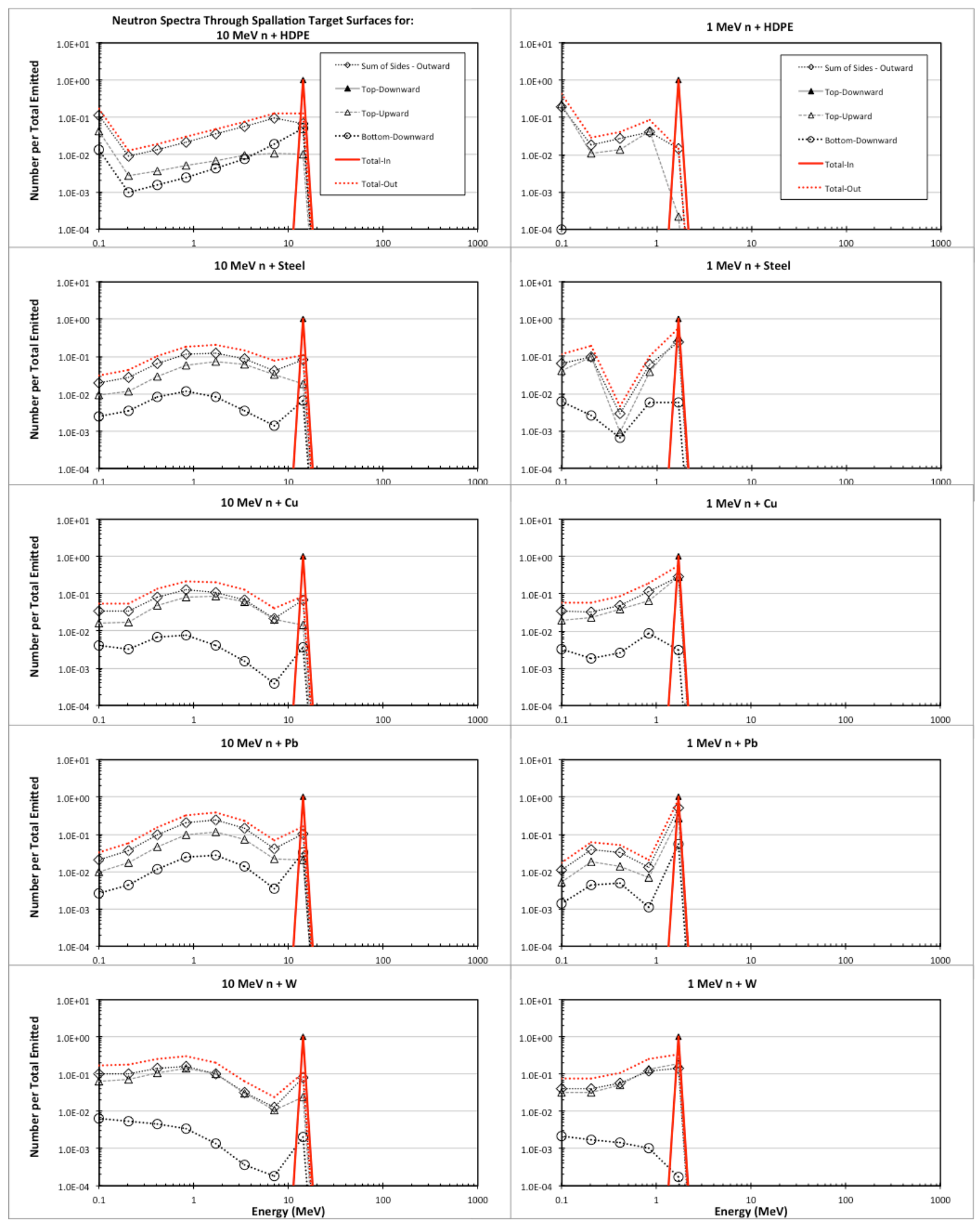

Figure 14-3. MCNP results for $10 \mathrm{MeV}$ and $1 \mathrm{MeV}$ monoenergetic neutrons on various materials.

Figure 14-4 shows examples from MCNPX of a $2.54 \mathrm{~cm}$ square "beam" of $1 \mathrm{GeV}$ and $100 \mathrm{MeV}$ neutrons incident at the center of the $30.8 \mathrm{~cm}$ cube of $\mathrm{Pb}$ for the resulting cascades of particles produced by the first 100 beam neutrons throughout the $\mathrm{Pb}$. The highest density of tracks is 
along the beam direction, though events occur throughout the material volume. The upper plots are for proton tracks (red lines), while the lower plots are for pion tracks (green lines). The left column is for $1 \mathrm{GeV}$ incident neutrons, and the right hand column is for $100 \mathrm{MeV}$ incident neutrons. The tracks are between the creation and termination points. The other (non-connected) points are for neutron collisions. There are far more tracks for $1 \mathrm{GeV}$ compared to $100 \mathrm{MeV}$, as expected.
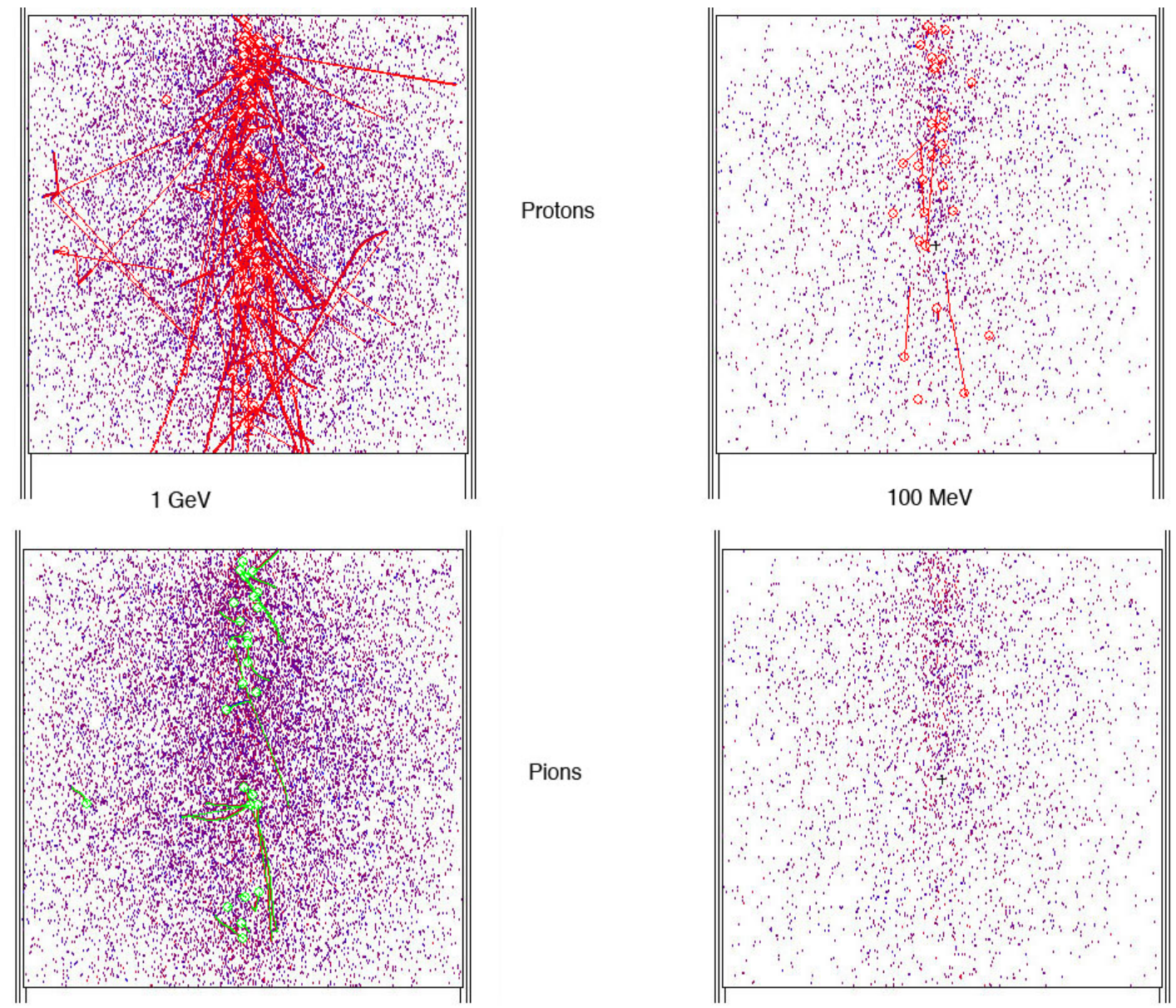

Figure 14-4. Simulation of collision points and cascade tracks for $1 \mathrm{GeV}$ (left) and $100 \mathrm{MeV}$ (right) neutron beams on $\mathrm{Pb}$.

Another result of the MCNP model was to predict the number of incoming neutrons that were not scattered at all during their passage through the cube of material. Table 14.2 shows these results. It is seen that only the high-energy neutrons have a small probability of not interacting in the cube of material. Over $90 \%$ of the cosmic flux interacts at least once in all materials.

Table 14.2. MCNP results percent of unscattered incident neutrons.

\begin{tabular}{|l|c|c|c|c|c|}
\hline Material & $\mathbf{1 ~ M e V}$ & $\mathbf{1 0} \mathbf{~ M e V}$ & $\mathbf{1 0 0} \mathbf{~ M e V}$ & $\mathbf{1 ~ G e V}$ & Cosmic Flux \\
\hline HDPE & $0 \%$ & $2 \%$ & $46 \%$ & $58 \%$ & $9 \%$ \\
\hline Steel & $0 \%$ & $0.03 \%$ & $0.8 \%$ & $4 \%$ & $0.9 \%$ \\
\hline Copper & $0.01 \%$ & $0.01 \%$ & $0.4 \%$ & $3 \%$ & $0.4 \%$ \\
\hline Lead & $0.6 \%$ & $0.6 \%$ & $1 \%$ & $5 \%$ & $0.7 \%$ \\
\hline Tungsten & $0 \%$ & $0.006 \%$ & $0.03 \%$ & $0.3 \%$ & $0.04 \%$ \\
\hline
\end{tabular}




\subsection{MCNP Cosmic Ray Spectra}

Turning to the cosmic ray neutrons, Figure 14-5 shows the normalized cosmic ray neutron spectra used in the MCNP modeling. The blue curve is the normalized differential flux (per unit area per second per $\mathrm{MeV}$ ), while the red curve shows the normalized flux (per unit area per second), which is the input spectrum entering the cube of material. The differential flux has the characteristic $\mathrm{E}^{-1}$ trend expected for cosmic rays. Similarly, Figure 14-6 shows the normalized cosmic ray proton spectra and Figure 14-7 shows the normalized cosmic ray muon spectra used in the MCNP modeling.

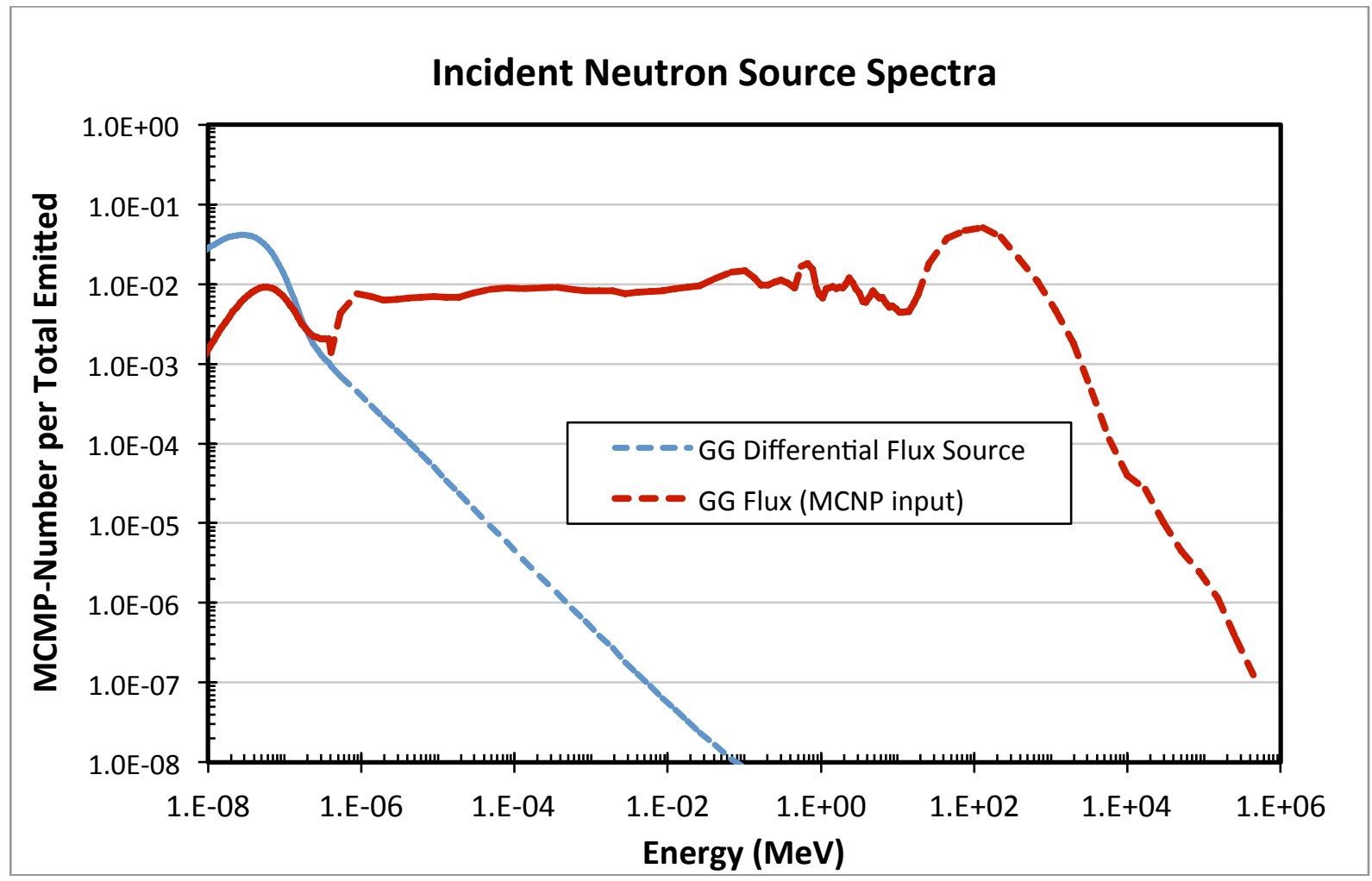

Figure 14-5. MCNP incident cosmic ray neutron spectra. 


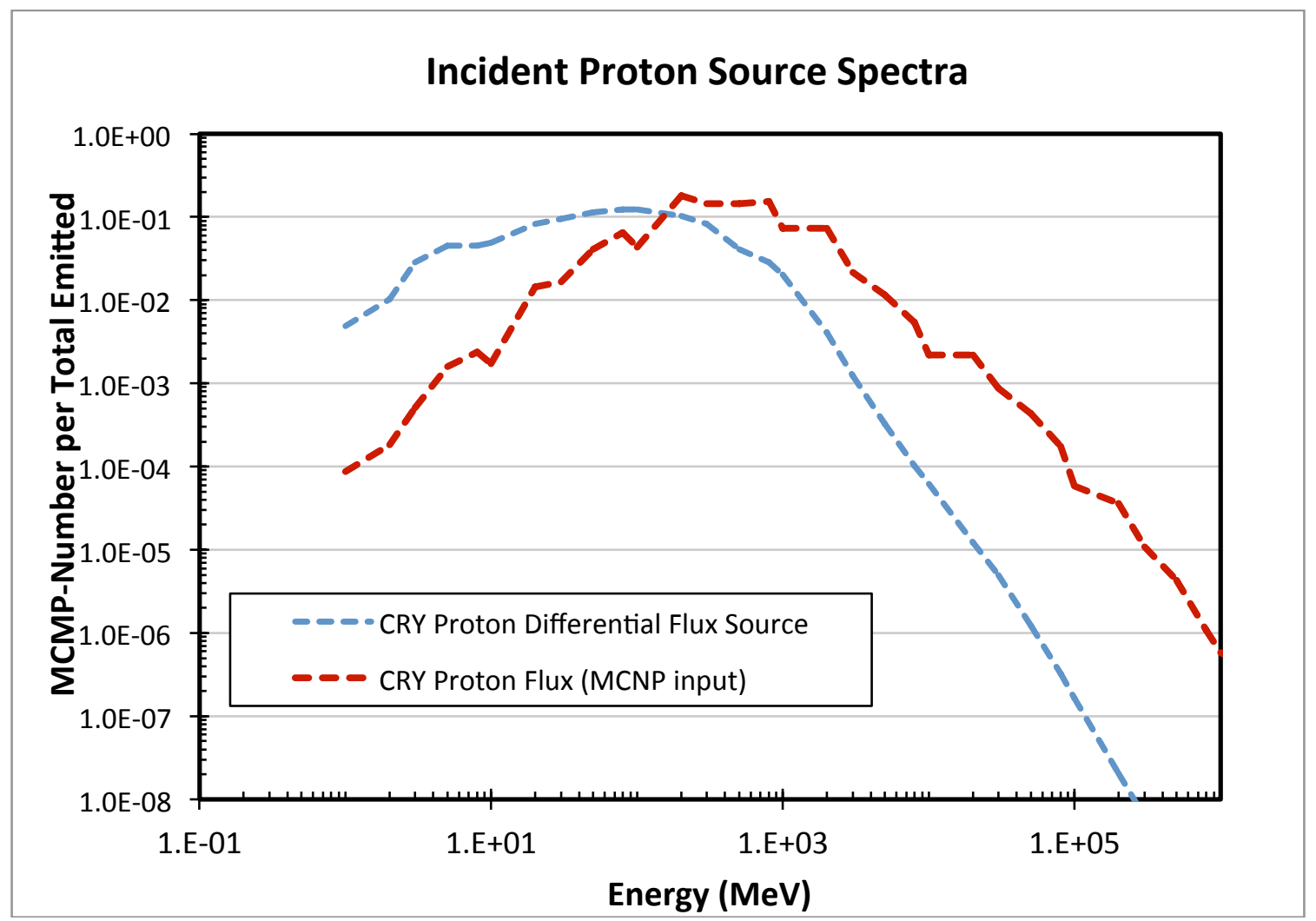

Figure 14-6. MCNP incident cosmic ray proton spectra.

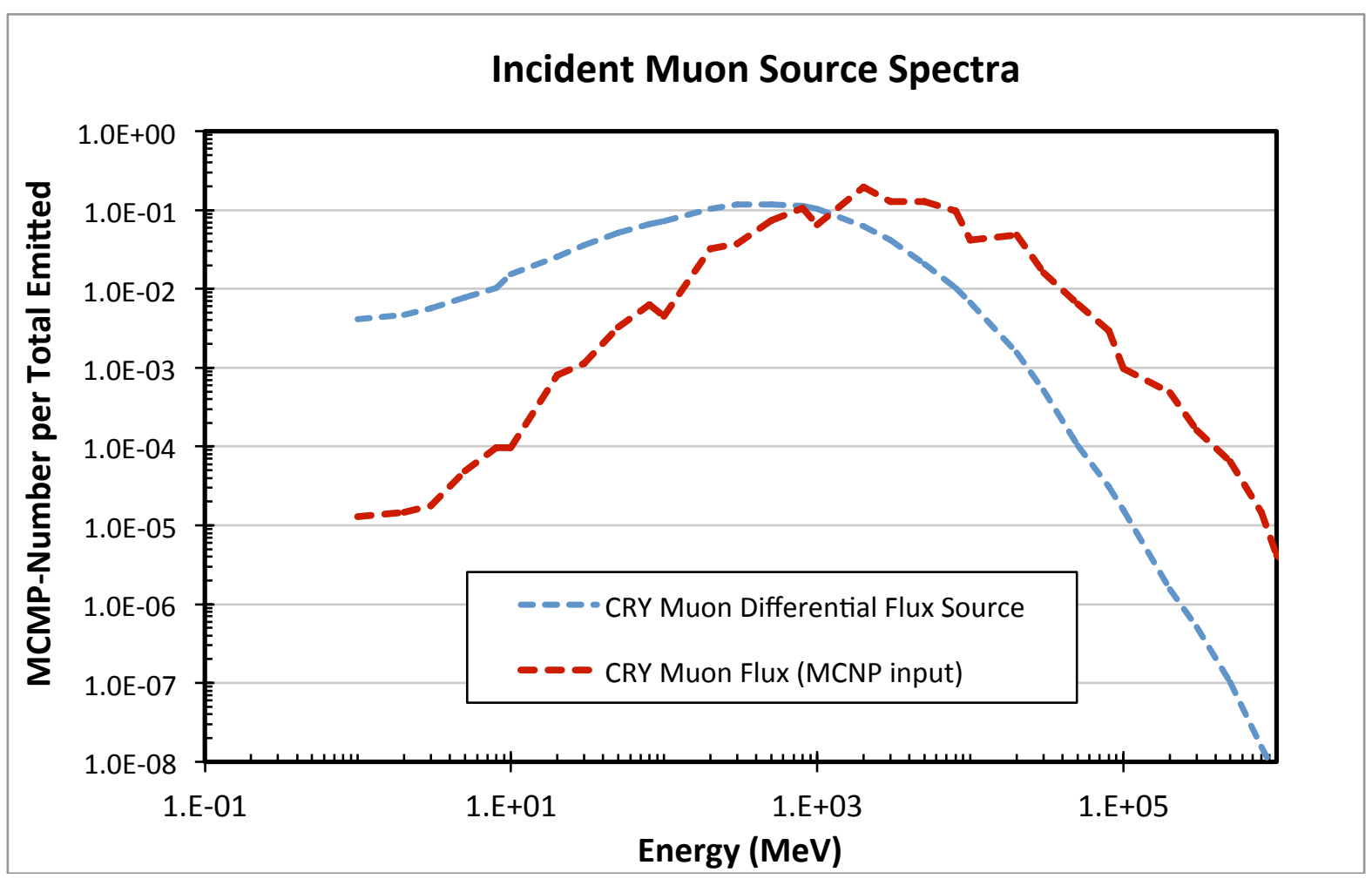

Figure 14-7. MCNP incident cosmic ray muon spectra. 


\subsection{MCNP Cosmic Neutron Results}

The MCNP model only looked at the total numbers of outgoing neutrons, and did not include a determination of multiplicity, which is a challenge to find with MCNP due to its statistical nature. Table 14.3 summarizes the results for neutrons, protons and muon spectra into the cube of material resulting in neutrons coming out of the cube of material. MCNP takes into account any absorption of neutrons in the material that would occur for low energy neutrons. The data is presented in terms of per incident particle. Also shown for protons and muons are the numbers scaled to the relative fluxes of these particles to neutrons in the sea level cosmic ray spectrum (see Section 1). The last column provides the percent contribution to the total number of outgoing neutrons from the incident muons and protons.

Table 14.3. MCNP results for total neutrons out for cosmic ray components on materials.

\begin{tabular}{|l|r|r|l|r|r|r|}
\hline & Meutrons Out/ & $\begin{array}{l}\text { Neutrons Out/ } \\
\text { Neutrons } \\
\text { Out Scaled } \\
\text { to Proton } \\
\text { Flux }\end{array}$ & $\begin{array}{l}\text { Neutrons Out/ } \\
\text { Nutrons In }\end{array}$ & $\begin{array}{l}\text { Neutrons } \\
\text { Out Scaled } \\
\text { to Muon } \\
\text { Flux }\end{array}$ & $\begin{array}{l}\text { \% } \\
\text { Contribution } \\
\text { of Muons } \\
\text { Plus Protons }\end{array}$ \\
\hline $\mathrm{HDPE}$ & 0.75 & 0.24 & 0.00 & 0.00 & 0.00 & $0.5 \%$ \\
\hline $\mathrm{Fe}$ & 1.35 & 3.34 & 0.05 & 0.11 & 0.14 & $12.1 \%$ \\
\hline $\mathrm{Cu}$ & 1.45 & 4.14 & 0.06 & 0.13 & 0.16 & $13.2 \%$ \\
\hline $\mathrm{Pb}$ & 2.36 & 9.47 & 0.13 & 0.22 & 0.28 & $14.6 \%$ \\
\hline $\mathrm{W}$ & 2.11 & 9.88 & 0.13 & 0.32 & 0.41 & $20.4 \%$ \\
\hline
\end{tabular}

The incident cosmic ray neutrons account for the bulk of the spallation events. The cosmic ray protons contribute from $0.4 \%$ to $5.1 \%{ }^{2}$ to the total number of outgoing neutrons depending on the material, with the largest contribution for the heaviest materials. The cosmic ray muons contribute from $0.1 \%$ to $15 \%$ to the total number of outgoing neutrons depending on the material, with the largest contribution for the heaviest materials. The size of the muon contribution is somewhat larger than was anticipated. Overall, protons and muons create up to $20.4 \%$ (for tungsten) of the outgoing neutrons for the geometry studies here.

Shown in Figure 14-8 are these data on the total outgoing neutron fluence per incident neutron, proton and muon as a function of neutron density for the various materials. The general trend is an increase in neutron multiplication with increasing density.

Shown in Figure 14-9 is the comparison of the Geant4 and MCNPX results for the total outgoing neutron fluence as a function of neutron density for the various materials. Both simulation approaches show a similar general trend, although there is a difference in the total number of neutrons (up to $\sim 50 \%$ ).

\footnotetext{
${ }^{2}$ Computed as $0.13 /(2.11+0.13+0.41)$
} 


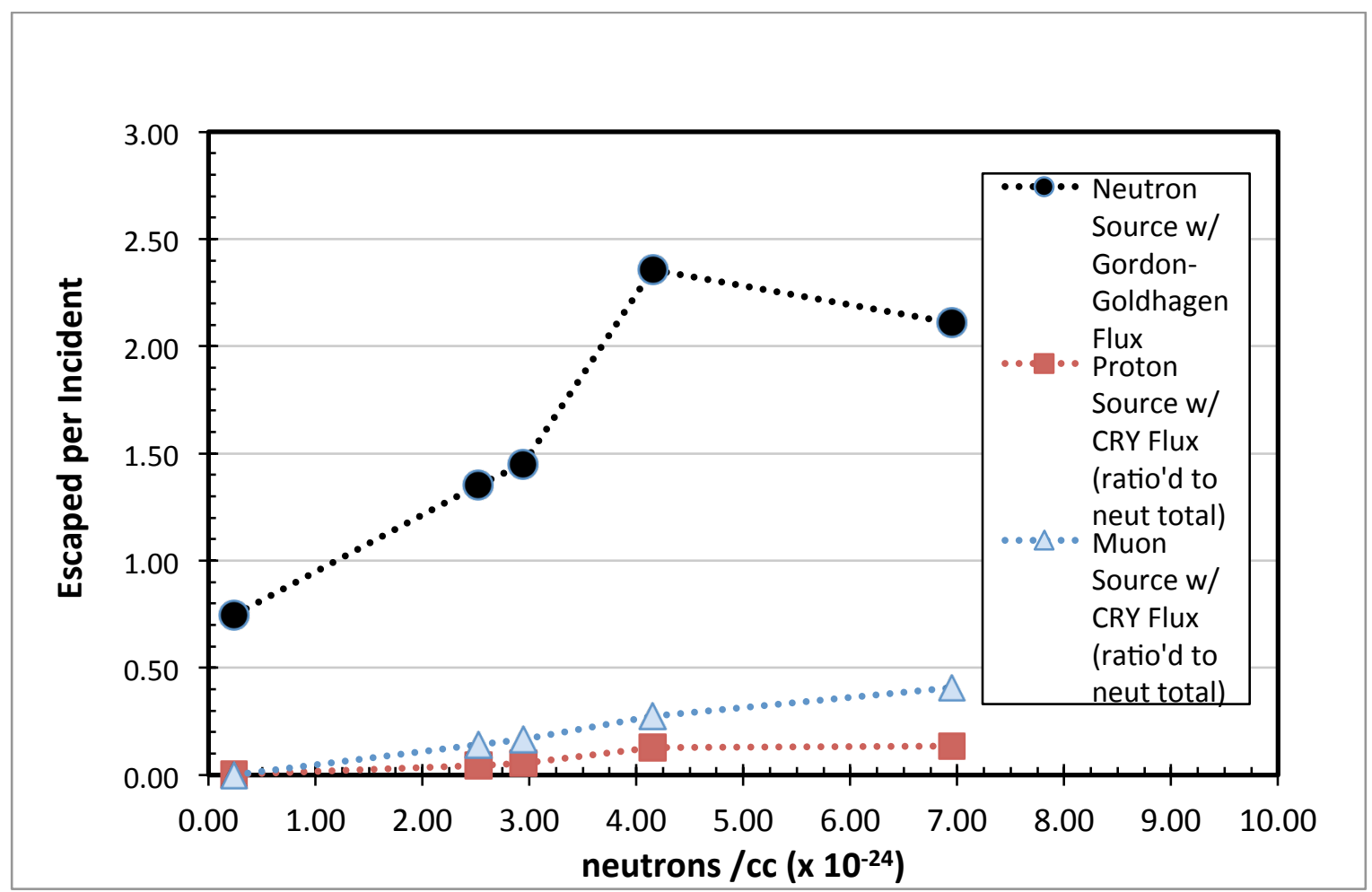

Figure 14-8. MCNP results for cosmic ray components for various materials.

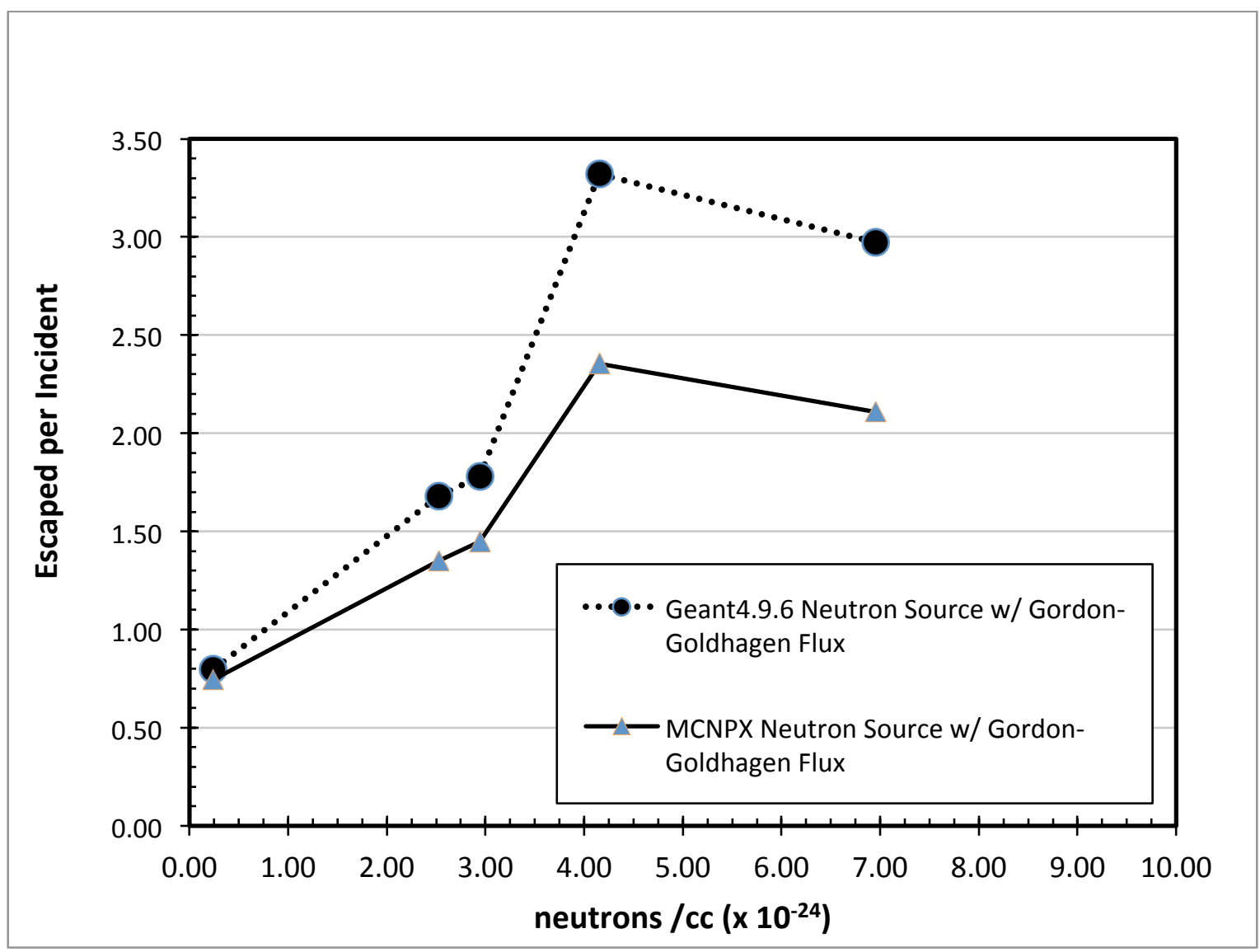

Figure 14-9. MCNPX and Geant4 results for total neutrons out for different materials. 


\subsection{MCNP Detection Results}

The MCNP model was extended to include the detector assembly. This was done in order to see if the detection efficiency depended upon the material type due to the variations in the energy spectra. Figure 14-10 shows a cutaway view from the MCNP model of interactions in the block and the detector for $\mathrm{Pb}$ and $\mathrm{Cu}$.

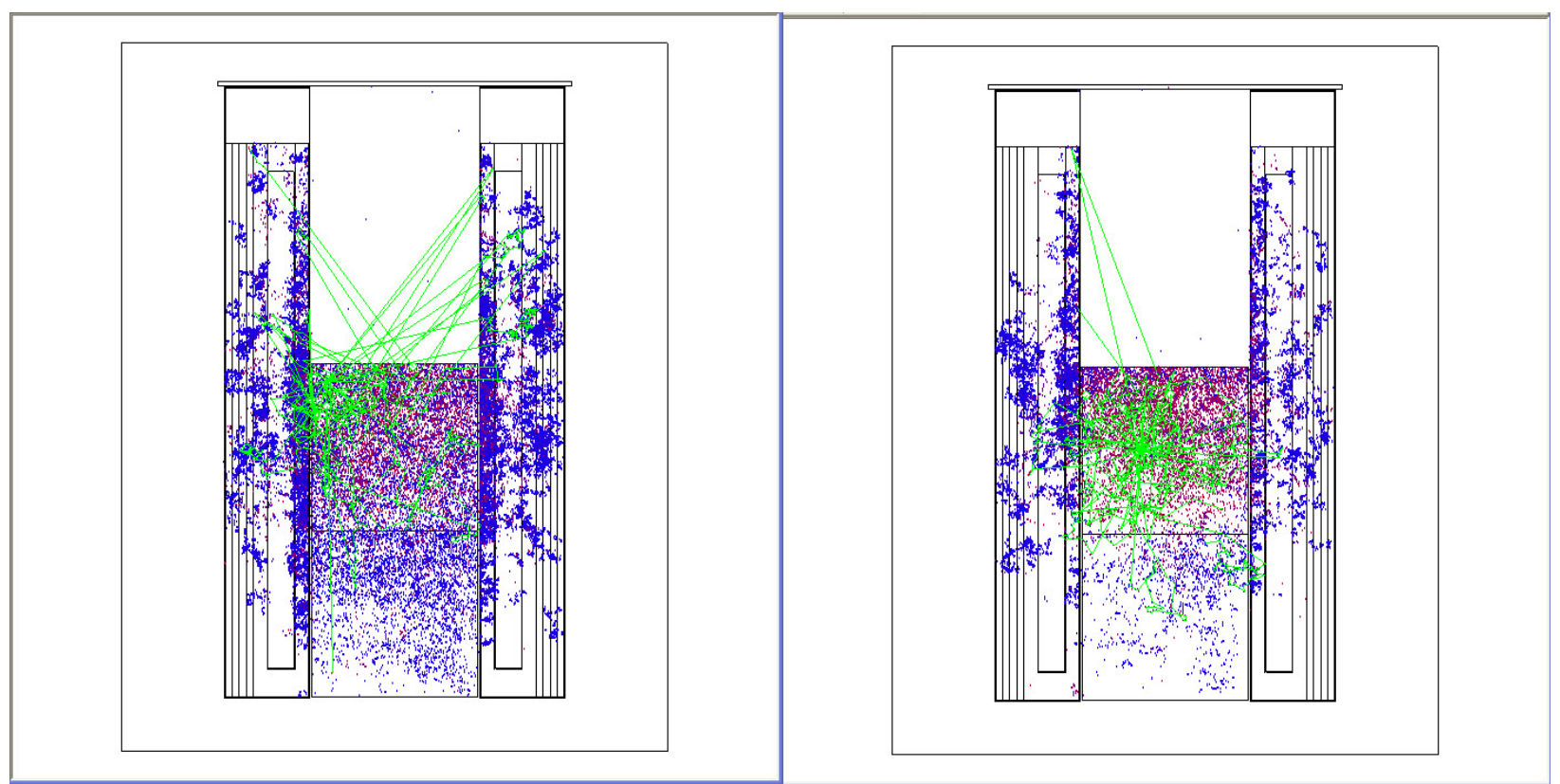

Figure 14-10. Cutaway view of the MCNPX detector model: $\mathrm{Pb}$ (left) and $\mathrm{Cu}$ (right).

Table 14.4 compares the ratio of neutrons out over neutrons in with the detector (column 3 ) to those without the detector (column 2). It can be seen that the detector increases the number of neutrons leaving the sample for all materials due to neutrons reflected back into the sample. The last column of Table 14.4 gives the overall detection efficiency for the incoming neutron spectrum (ratio of number detected to number incoming). The apparent increase in efficiency with density is due to the multiplication in the dense materials.

Table 14.4. MCNP results for total neutrons out for cosmic ray components on materials.

\begin{tabular}{|l|r|r|r|}
\hline Material & $\begin{array}{l}\text { Neutrons Out/ } \\
\text { Neutrons In } \\
\text { without Detector }\end{array}$ & $\begin{array}{l}\text { Neutrons Out/ } \\
\text { Neutrons In } \\
\text { with Detector }\end{array}$ & $\begin{array}{l}\text { MCNP \% of } \\
\text { Incoming } \\
\text { Detected }\end{array}$ \\
\hline $\mathrm{HDPE}$ & 0.75 & 1.06 & $9.9 \%$ \\
\hline $\mathrm{Al}$ & 1.16 & 1.72 & $20.6 \%$ \\
\hline $\mathrm{Fe}$ & 1.35 & 1.81 & $27.4 \%$ \\
\hline $\mathrm{Cu}$ & 1.45 & 1.89 & $30.3 \%$ \\
\hline $\mathrm{Pb}$ & 2.36 & 3.88 & $52.5 \%$ \\
\hline $\mathrm{W}$ & 2.11 & 2.53 & $43.6 \%$ \\
\hline
\end{tabular}


Figure 14-11 shows the total number of neutrons exiting the material block as a function of neutron density with and without the detector divided by the number into the material. This includes neutrons reflected back into the material by the detector for the with detector case. The lines are to guide the eye. The figure shows that the number of neutrons going out of the material, and the detected neutrons, generally track each other. The enhancement of neutrons out for all materials when the detector is present is likely due to neutrons reflected back into the material by the detector that then produce more spallation events. The figure also shows the percent detected, defined as the number of neutrons detected divided by the number into the material block, including all neutron multiplication effects. Neutrons reentering $\mathrm{Pb}$ apparently have a higher probability of producing additional spallation. This will tend to enhance the multiplicity number for $\mathrm{Pb}$ relative to other materials. The effect is not as large for $\mathrm{W}, \mathrm{so} \mathrm{Pb}$ is unique in some way.

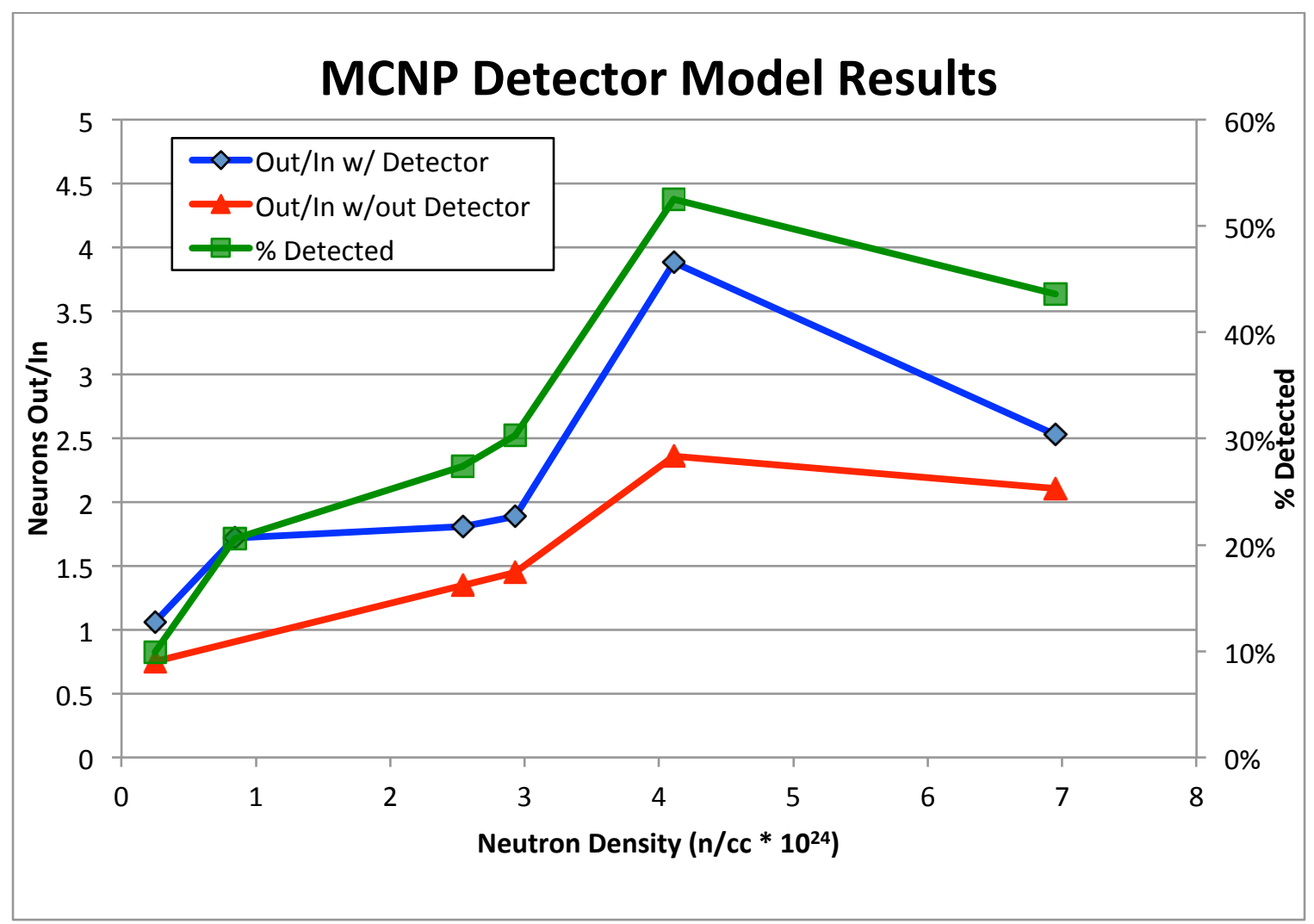

Figure 14-11. MCNPX results versus neutron density for neutrons out and neutrons detected with the detector in place.

Figure 14-12 shows the same data versus atomic mass (A), with the addition of a line showing $\mathrm{A}^{0.73}$. This is the reported approximate cross section dependence given by Barabanov et al. [2006]. The data follows the general trend, but has significant scatter. 


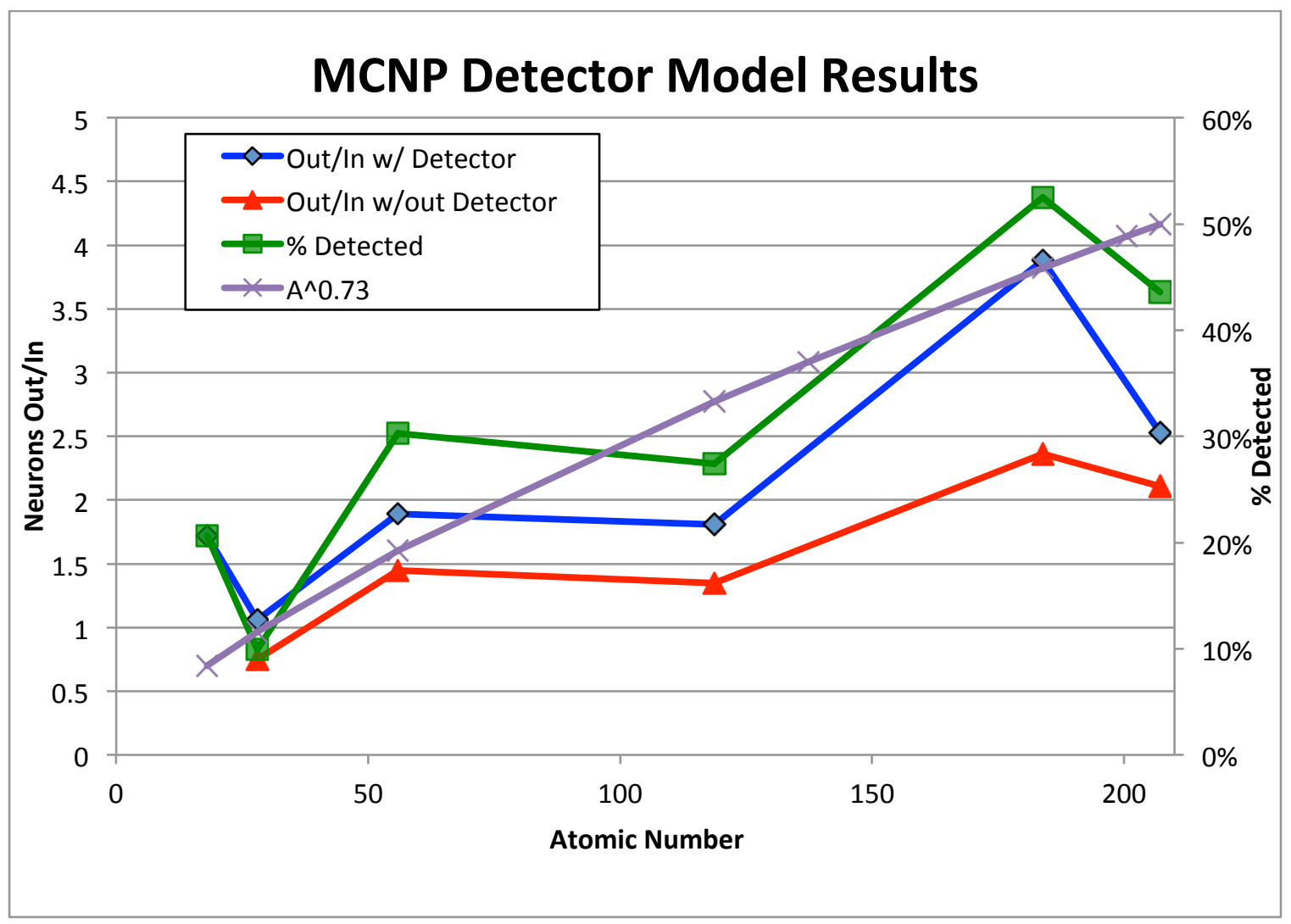

Figure 14-12. MCNPX results versus atomic mass for neutrons out and neutrons detected with the detector in place. 


\section{Discussion of Multiplicity Results}

Figure 15-1 shows a direct comparison of the Geant4 Monte Carlo model results (for net counts above a Poisson distribution) with the measurement results for net counts greater than the Poisson distribution, where the measurement data has been scaled to match the $\mathrm{Pb}$ model value since the model scale is arbitrary. The detector response is not folded in the presented simulated results, so effects such as detector efficiency are not included in the simulated data presented in this section. The $1 \mathrm{~ms}$ time window experimental data was used for the comparison, and the 0.1 ms data produces a similar result. The errors in the values shown are smaller than the markers. The HDPE result is consistent since it is about zero, and the $\mathrm{W}$ experiment is also similar to the simulation. However, the $\mathrm{Al}, \mathrm{Fe}$ and $\mathrm{Cu}$ results do not agree with the model, predicting a higher rate by about a factor of two to three for $\mathrm{Cu}$ and $\mathrm{Fe}$, and 25 for Al. Table 15.1 provides the data that is plotted in Figure 15-1 (normalized to the $\mathrm{Pb}$ value).

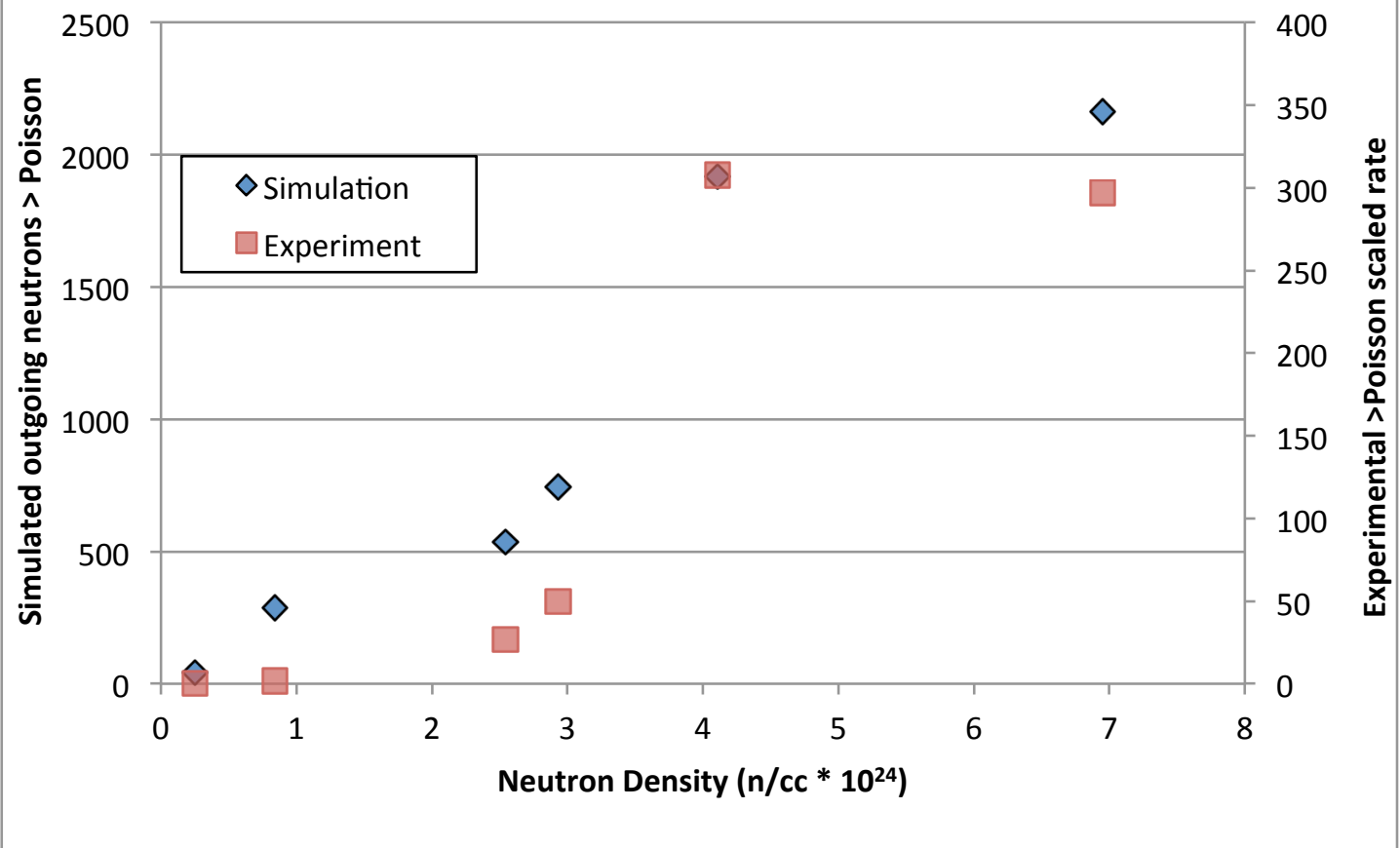

Figure 15-1. Comparison of simulation to measurement for counts greater than Poisson in a $1 \mathrm{~ms}$ window.

Table 15.1. Data for various materials shown in Figure 15-1.

$\begin{array}{lcrll}\text { Material } & \begin{array}{c}\text { Neutron Density } \\ (\mathbf{n} / \mathbf{c c} \text { x 10-24) }\end{array} & \begin{array}{c}\text { Model Counts }> \\ \text { Poisson }\end{array} & \begin{array}{c}\text { Ratio Exp/Model } \\ \text { Normed to Pb }\end{array} \\ \text { Polyethylene } & 0.25 & 42 & 0.00 \\ \text { Aluminum } & 0.84 & 286 & 0.04 \\ \text { Steel } & 2.54 & 536 & 0.31 \\ \text { Copper } & 2.93 & 744 & 0.42 \\ \text { Lead } & 4.11 & 1917 & 1.00 \\ \text { Tungsten } & 6.95 & 2163 & 0.86\end{array}$


The direct comparison between the model and experiment is complicated since detection efficiency has not been taken into account as a function of multiplicity. The detection "efficiency" for the model is $100 \%$, whereas the detection efficiency for the experiment is of order $\sim 17 \%$ for each neutron (this is the fission spectrum efficiency, and is different for the spallation spectrum efficiency. The MCNP models included the detector system in the model, but not the Geant4 models. A correction factor has to be applied to the experimental data (or the model results) based on the theoretical detection efficiency. This correction factor must take into account the efficiency for detecting the number of neutrons that are available to detect. The comparison shown in Figure 15-1 does not include a material dependent correction.

Figure 15-2 shows another comparison to simulation of the measured multiplicity found with the $\mathrm{R}+\mathrm{A}$ method for the materials studied in this work as a function of neutron density. The experimental triples and quads from the $\mathrm{R}+\mathrm{A}$ analysis are compared to the simulation of counts greater than a Poisson distribution, showing a similar trend with neutron density. The tungsten data was scaled by volume to be comparable to the other materials. The comparison using this method shows agreement for $\mathrm{W}$ and $\mathrm{Pb}$, but again, the $\mathrm{Fe}$ and $\mathrm{Cu}$ experimental values are about a factor of two lower than the simulation, and $\mathrm{Al}$ and polyethylene did not yield any significant experimental results above zero even though the model predicts some.

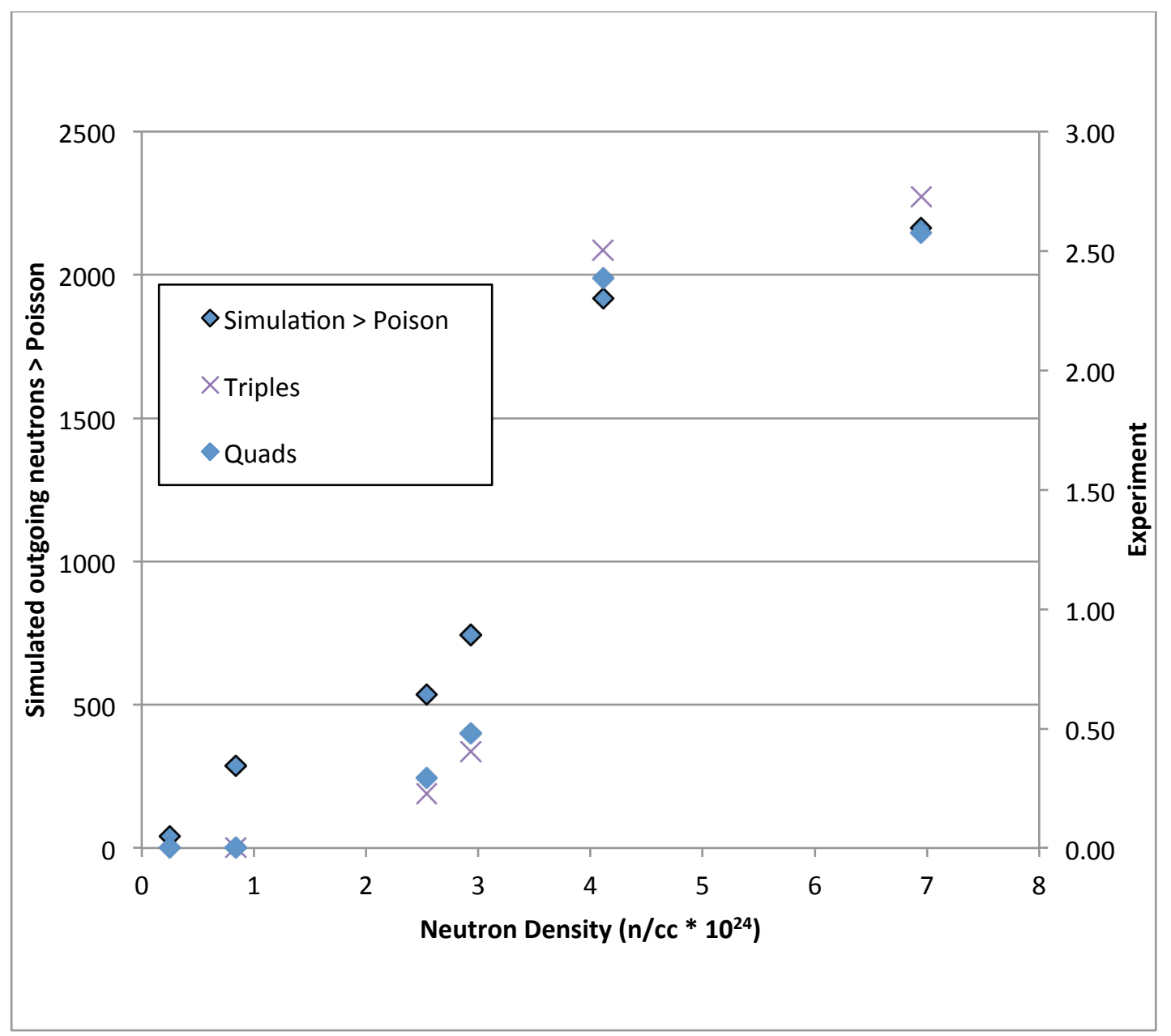

Figure 15-2. Comparison of $\mathrm{R}+\mathrm{A}$ analysis of measurements to simulation of counts greater than Poisson in a $1 \mathrm{~ms}$ window. 
These results for multiplicity indicate that there is a discrepancy between the model results and the measurements for the lighter materials. Since the measurements were in a controlled geometry and the data rates, and resulting dead time, were small, there is no obvious problem with the measurements, except that the tungsten measurement had to scaled to match the geometry of the rest of the studied materials. With regard to the model, there are several possible explanations: the incoming neutron spectrum might be different than assumed, the cross-section libraries might be wrong between materials, or simplification of the model might cause the observed differences.

The origin of this discrepancy requires further investigation: is there a problem with the measurements or with the models? If this disagreement is correct, the model over predicts the interaction rate for lower density materials ( $\mathrm{Al}, \mathrm{Fe}$, and $\mathrm{Cu}$ ), and thus the effectiveness of iron and copper shielding on the incoming neutrons could be substantially smaller than currently assumed. There are differences in the neutron spectra that come from spallation in the various materials, as discussed, but these spectra are similar in that much of the neutron flux is below one $\mathrm{MeV}$ where the detection efficiency is high. The development of targets for spallation neutron source facilities has developed a good basis for heavy target production rates used in the simulations; however, lighter materials like aluminum, iron and copper may not have validated cross sections for the cosmic energies studied here. Experimental measurements of neutron energy spectra exiting thick targets of these materials should be made with incident spectra similar to that from cosmic rays. 


\section{Conclusions}

This paper has reported on multiplicity measurements for a range of materials and compared them to Monte-Carlo modeled results based on the Geant4 and MCNPX simulation codes. Measurements of neutron multiplicity were performed using a neutron coincidence counter with a list mode data acquisition system that recorded the time of each detected neutron. Post analysis was performed to extract the detected multiplicity. Measurements were made on polyethylene, aluminum, iron, copper, lead and tungsten.

The evaluation reported here was undertaken to study the fidelity of Monte Carlo models to simulate spallation neutron yields from different shielding materials induced by cosmic-rays. This was motivated by the large difference between shield model results from two different simulations. Geant 4 predicted a greater degree of shielding by the iron shipping shield than the SHIELD code by a factor of $\sim 2.5$. This difference is what motivated the current study to see how well a model could predict experimental results.

The result of this study is that the relative multiplicity per event extracted from the models are in agreement for dense materials, but disagree with the experimental findings by a factor of more than two for the lower density materials $\mathrm{Al}, \mathrm{Fe}$ and $\mathrm{Cu}$. The model over-predicts the effect of $\mathrm{Fe}$ shielding by about a factor of three, making the experimental results more consistent with the SHIELD code predictions. This might be an artifact of the way the neutron data was obtained, or it may represent limitations in the models for low-density materials. A number of experimental tests were made indicating that the experimental results are probably correct, which points to the model results as being the more likely problem.

Two Monte Carlo approaches, Geant4 and MCNPX, were compared to each other to confirm the reproducibility of the experimental results with different independently developed codes. The last official release of MCNPX (version 2.70) did not provide a tally option for tabulating the multiplicities of the target materials studied, so an alternative simulation scenario was devised for this inter-code comparison. Several simulation runs involving mono-energetic neutrons in the range of interest for cosmic ray studies were performed and the result of the inter-code comparison is an agreement by better than $\sim 30 \%$ in the total neutron yield.

The scope of this work was not to reproduce accurately the experimental set-up, but simply to evaluate the models' behavior when switching between different shielding materials. This result shows that the Geant4 Monte Carlo model results follow the correct trend as determined by experiment for total neutron yield and multiplicity. The predicted multiplicity as a function of target material increased with material density, with an approximately linear relationship. This indicates that Geant 4 can provide adequate predictions for the spallation effects of cosmic ray neutrons, with the caveat that spallation rates for lower density materials are over-predicted by the models.

The MCNP models showed that only a small percentage of the incident cosmic neutron flux is not scattered in a cubic foot of target material, being 9\% for HDPE and decreasing to $0.04 \%$ for tungsten. The MCNP models also looked at the contributions from cosmic proton and muon secondaries on the Earth's surface. It was predicted that these two components could contribute $12 \%$ to the outgoing neutrons for iron, increasing up to $20 \%$ for tungsten.

These theoretical tools must be used with care to correctly simulate shielding techniques for the next generation of low-background experiments and to estimate background rates. Unless 
benchmark measurements are made to validate Monte Carlo modeling for each application, there use is limited to shielding comparison studies, not as a predictor of absolute shielding efficiency.

Future measurements should be performed of neutron spectra exiting large quantities of materials, especially the lower density materials where there remains a large uncertainty in the ability of the models to correctly predict the effects of such materials. 


\section{Acknowledgements}

This work was supported by the United States Department of Energy Office of Science Office of Nuclear Physics. Pacific Northwest National Laboratory is operated for the United States Department of Energy under contract DE-AC05-76RLO 1830. 


\section{References}

Aalseth CE, et al. (MAJORANA collaboration). 2009. "The MAJORANA DEMONSTRATOR: An R\&D project towards a tonne-scale germanium neutrinoless double-beta decay search." The American Institute of Physics, Conf. Proc. 1182 pp 88-91.

Aguayo Navarrete E, RT Kouzes, AS Ankney, JL Orrell, TJ Berguson, and MD Troy. 2011. "Cosmic Ray Interactions in Shielding Materials." PNNL-20693, Pacific Northwest National Laboratory, Richland, WA

Aguayo E, JE Fast, RT Kouzes, JL Orrell. 2112a. "The $\mu$-Witness detector: A ruggedized, portable, flux meter for cosmogenic activation monitoring." Transactions on Nuclear Science.

Aguayo E, RT Kouzes, JL Orrell, DJ Reid, JE Fast. 2012b. “Optimization of the Transport Shield for Neutrinoless Double-beta-decay Enriched Germanium.” PNNL Technical Report PNNL-21302.

Agostinelli S, et al. 2003. "Geant4-a Simulation Toolkit." Nuclear Instruments \& Methods in Physics Research Section a-Accelerators Spectrometers Detectors and Associated Equipment 506(3):250-303. 10.1016/s0168-9002(03)01368-8.

Allkofer OC, K Carstensen, WD Dau, H Jokisch. 1975. "The Absolute Cosmic Ray Flux at Sea Level.” J Phys. G: Nucl. Phys. 1, No. 6. L51-L52.

Armstrong TW, KC Chandler, J Barish. 1973. Journal of Geophysical Research 78 pp. 27152726.

Barabanov, I, S Belogurov, L Bezrukov, A Denisov, V Kornoukhov, and N Sobolevsky. 2006. "Cosmogenic Activation of Germanium and Its Reduction for Low Background Experiments." Nuclear Instruments and Methods in Physics Research Section B: Beam Interactions with Materials and Atoms 251(1):115-20.

Crane TW and MP Baker. 1991. Chapter 13 in "Neutron Detectors," in Passive Nondestructive Assay of Nuclear Materials, edited by T. D. Reilly, N. Ensslin, and H. A. Smith, US Nuclear Regulatory Commission NUREG/CR-5550.

Ensslin N, WC Harker, MS Krick, DG Langner, MM Pickrell and JE Stewart. 1998.

"Application Guide to Neutron Multiplicity Counting." Los Alamos National Laboratory Technical Report LA-13422-M.

Grieder PKF. 2001. “Cosmic Rays at Earth.” Elsevier, Amsterdam.

IS, JR Hook, IA Jenkins, KE Turver. 1974. "Momentum Spectra Of Nuclear Active Particles In Cosmic-Radiation At Sea-Level .1. Experimental-Data.” Journal Of Physics A-Mathematical And General 7, Issue 6, pp. 741-764

GE Reuter Stokes Measurements Solutions (Twinsburg, OH).

Gordon MS, P Goldhagen, KP Rodbell, TH Zabel, HHK Tang, JM Clem, and P Bailey. 2004. "Measurement of the Flux and Energy Spectrum of Cosmic ray Induced Neutrons on the Ground." IEEE Transactions On Nuclear Science, Vol. 51, No. 6; and private communication of data from P Goldhagen. 
Greisen KI. 1942. "The Intensities Of The Hard And Soft Components Of Cosmic Functions Of Altitude And Zenith Angle.” Phys. Rev. 61, pp. 212-221.

Hagmann C, D Lange, D Wright. 2008. "Cosmic ray Shower Library (CRY)." Technical Manual, 5 pages. Lawrence Livermore National Laboratory, Livermore, CA. http://nuclear.llnl.gov/simulation/cry.pdf.

Geant4. 2011. The Geant4 Collaboration. Physics Lists - Use Cases. Accessed on July 22, 2011 at http://geant4.org/geant4/support/physicsLists/referencePL/useCases.shtml (last updated July 9, 2011).

Hess WN, HW Patterson, R Wallace, EL Chupp. 1959. "Cosmic ray Neutron Energy Spectrum." Physical Review 116 pp 445-57.

Kouzes RT, JH Ely, A Seifert, ER Siciliano, DR Weier, LK Windsor. 2008. “Cosmic-RayInduced Ship-Effect Neutron Measurements And Implications For Cargo Scanning At Borders." Nuclear Instruments And Methods In Physics Research A 587 Pp. 89-100.

MCNP. 2003. X-5 Monte Carlo Team, MCNP - A General Purpose Monte Carlo N-Particle Transport Code, Version 5, Los Alamos National Laboratory Report LA-UR-03-1987, updated 2005.

Pelowitz DB (ed.). 2011. “MCNPX User's Manual”, Version 2.7.0. Los Alamos National Laboratory Report LA-CP-11-00438.

Prael RE and H Lichtenstein. 1989. "Users Guide to LCS: The LAHET Code System.” Los Alamos National Laboratory Report LA-UR-89-3014.

Prael RE. 1994. "A Review of the Physics Models in the LAHET Code." Los Alamos National Laboratory Report LA-UR-94-1817.

Switzer T, RR Hansen, RT Kouzes, RJ Arthur, WK Pitts. 2003. "Neutron Detection Authentication Case Study." Technical Report PNNL-14222

Ziegler JF. 1998. "Terrestrial Cosmic Ray Intensites." IBM Journal of Research and Development 42(1):117. 


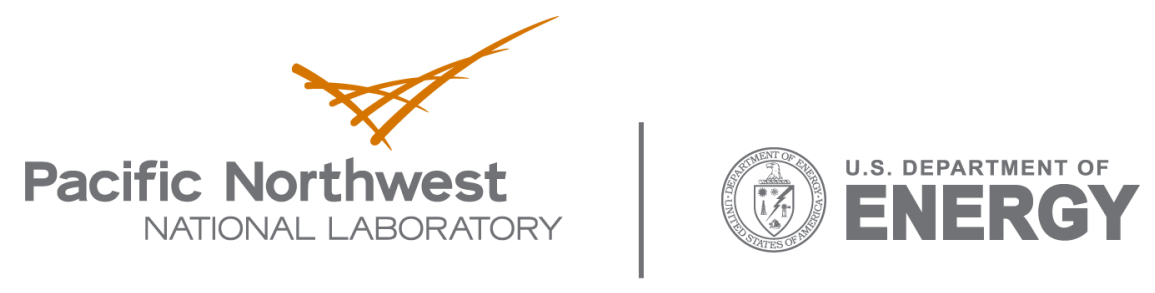

902 Battelle Boulevard

P.O. Box 999

Richland, WA 99352

1-888-375-PNNL (7665)

www.pnl.gov 